\title{
Diagrammatic strong coupling expansion of a U(1) lattice model in the Fourier basis
}

\author{
Afsaneh Kianfar and Amir H. Fatollahi๑* \\ Department of Physics, Faculty of Physics and Chemistry, Alzahra University, Tehran 1993891167, Iran
}

(Received 20 May 2021; accepted 11 October 2021; published 15 November 2021)

\begin{abstract}
The transfer-matrix of the U(1) lattice gauge theory is investigated in the field Fourier space, the basis of which consists of the quantized currents on lattice links. Based on a lattice version of the current conservation, the transfer-matrix elements are shown to be nonzero only between current states that differ in circulating currents inside plaquettes. In the strong coupling limit, a series expansion is developed for the elements of the transfer matrix, to which a diagrammatic representation based on the occurrence of virtual link and loop currents can be associated. With $g$ as the coupling, the weight of each virtual current in the expansion is $1 / g^{2}$, by which at any given order the relevant diagrams are determined. Either by interpretation or through their role in fixing the relevant terms, the diagrams are reminiscent of the Feynman ones of the perturbative small coupling expansions.
\end{abstract}

DOI: 10.1103/PhysRevD.104.094506

\section{INTRODUCTION}

According to the perturbative formulation of quantum field theories, the transition amplitudes of physical processes are expressed by series expansions in the small coupling constant. Whenever applicable, the perturbative series makes it possible to calculate the transition rates up to the desired accuracy. Based on the interpretation that the expansion terms are representing the space-time virtual events between the initial and final states [1], the perturbative expansions are commonly represented by a set of graphs: the Feynman diagrams. Apart from pure theoretical interests, diagrammatic representations have found a crucial role in determining and managing the relevant terms at any given order of the coupling constant. As an example of the role of Feynman diagrams in managing the perturbative expansions, representatives of 891 diagrams contributing to the anomalous magnetic moment of leptons at the order $e^{8}$ are presented in Fig. 1 [2].

The main purpose of the present work is to introduce a diagrammatic expansion for lattice gauge theories $[3,4]$ in the strong coupling regime. In particular, we consider the transfer matrix of the $\mathrm{U}(1)$ lattice gauge theory in the field Fourier space. In the common approach, the elements of the transfer matrix are defined in the field space through the

\footnotetext{
* Corresponding author. fath@alzahra.ac.ir

Published by the American Physical Society under the terms of the Creative Commons Attribution 4.0 International license. Further distribution of this work must maintain attribution to the author(s) and the published article's title, journal citation, and DOI. Funded by SCOAP ${ }^{3}$.
}

Euclidean action between two adjacent times of the discrete space-time. Due to the angle-variable nature of the gauge fields in the lattice formulation, the Fourier conjugates of fields turn out to be integer valued, being identified as quantized currents on lattice links. It is for the matrix elements between these currents that the series expansion in the strong coupling and associated diagrammatic representation are derived. With $g$ as the gauge coupling, the expansion parameter is $1 / g^{2}$, which is small in the strong coupling limit. As we will see in detail, the expansion of matrix elements between two current-states is interpreted as the occurrence of all possible virtual link and loop currents that transform the current states to vacuum (the state with no current). In this sense, the diagrammatic expansion may be considered as a "current expansion." The weight of each virtual current is $1 / g^{2}$, which serves as the expansion parameter. Either by interpretation or through managing the relevant terms in a given order of the strong coupling expansion, the diagrams play the role of Feynman diagrams at the small coupling regime.

The use of a Fourier basis in lattice gauge models dates back almost to the time of appearance of these models. The transform of plaquette degrees to Fourier ones known as dual variables [5], is used to qualitatively describe the underlying mechanism governing the phases of the U(1) model in three and four dimensions [6]. In particular, performing the dual variable transform in the partition function and using a certain small coupling model known as Villain action [7], the U(1) model is reduced to that of a gas of monopoles or monopole loops [6]. The dual formulation of U(1) lattice model shows also clear advantages for various numerical purposes; see Ref. [8] for a recent review. The advantages include the replacement of 


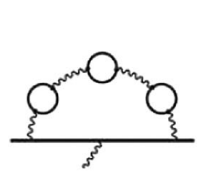

I(a)

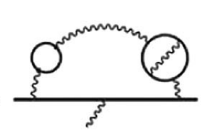

I(b)

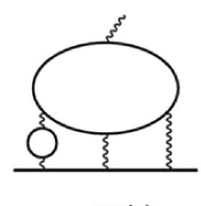

IV(a)

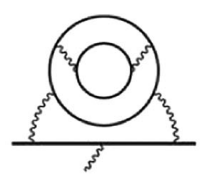

I(c)

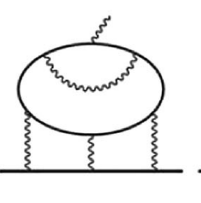

$\operatorname{IV}(\mathrm{b})$

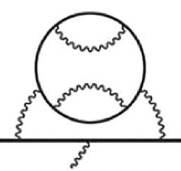

I(d)

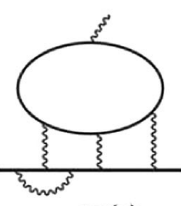

IV(c)

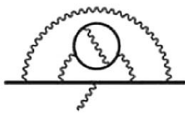

II(a)

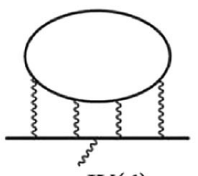

IV(d)

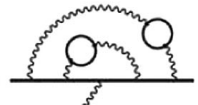

II(b)

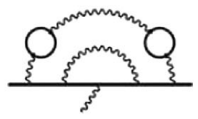

II(c)

FIG. 1. Thirteen representatives of 891 Feynman diagrams contributing the lepton's $g-2$ at the order $\alpha^{4}$. Reprinted from [2].

the continuous variables by the more tractable integer ones, and also the more efficient and accurate calculation of expectation values in the presence of multiple or separated source charges [9-13].

The present strong coupling expansion is different in two respects to that of the perturbative approach, in which the small coupling expansion is associated with the elements of the $S$ matrix between initial and final states, usually with an infinite time separation. First, the present strong coupling expansion is between current states at two adjacent times, as the transfer-matrix elements are defined based on the Euclidean action between the adjacent times. The second difference is related to the fact that, the $S$-matrix element between equal states is not zero but supposedly infinite. In the present expansion, however, the matrix-element between equal current states other than vacuum is vanishing in the extreme strong coupling limit $1 / g^{2} \rightarrow 0$. As will be discussed in detail, this simply is related to the fact that, for a nonzero result, the Fourier integrals related to initial and final states are to be nonzero independently. This promotes the vacuum state as a seemingly passed intermediate state in all transitions.

A manageable strong coupling expansion for the elements of the transfer matrix provides a basis to find the energy spectrum of the model in the strong coupling limit, especially when it is combined with numerical calculations.

The present diagrammatic expansion in the strong coupling limit is based on Ref. [14], in which the elements of the transfer matrix were obtained in the Fourier basis through the plaquette-link matrix. Accordingly, it is observed that by a lattice version of current conservation, emerged through the construction, the transfer-matrix elements are nonzero only between current states that differ in loop currents circulating in one or more plaquettes. By the mentioned version of current conservation, it is found that the transfer matrix is block diagonal in the Fourier basis [14]. The current states differing only in loop currents belong to the same block.

The paper is organized as follows. In Sec. II, a short review of the formulation of the transfer matrix in the field Fourier basis is presented, in line with [14]. In Sec. III, a detailed description of the emerged notions in the Fourier basis is discussed. In particular, the exact connection between current states belonging to the same block is presented. In Sec. IV, first some examples of the strong coupling expansions and then their elementary graphical representations are presented, and then rules are set up for diagrammatic expansions. Section V presents the detailed application of the given rules at higher orders in several examples. In Sec. VI the present expansion is used to calculate the ground state and some excited energies of the model in the strong coupling regime. In Sec. VII, based on the observation that the lattice size dependence of expansions can be factored out from the matrix elements and eigenvalues, the physical interpretation of the results is discussed. Section VIII is devoted to concluding remarks. Some derivations and extended expressions, as well as more examples of the application of expansion rules are presented in Appendices A, B, and C.

\section{REVIEW: TRANSFER MATRIX IN FOURIER BASIS}

In Ref. [14], a formulation of the pure U(1) lattice gauge theory in the field Fourier basis is presented. In particular, based on the plaquette-link matrix the elements of the transfer-matrix $\hat{V}$ in the Fourier basis are obtained explicitly, for which some mathematical statements are expressed [14]. In this section, a short review of the mathematical derivation of matrix elements is presented.

Following Refs. [15,16], the formulation is presented in the temporal gauge $A^{0} \equiv 0$, in which the transfer matrix takes a simple form. The link at site $\boldsymbol{r}$ in spatial direction " $i$ " is represented by $(\boldsymbol{r}, i)$. Conveniently, the gauge variables on the spatial link $(\boldsymbol{r}, i)$ at adjacent times $n_{t}$ and $n_{t}+1$ are replaced by the angle variables:

$$
\begin{gathered}
\theta^{(\boldsymbol{r}, i)}=a g A_{n_{t}}^{(\boldsymbol{r}, i)}, \\
\theta^{\prime(\boldsymbol{r}, i)}=a g A_{n_{t}+1}^{(\boldsymbol{r}, i)},
\end{gathered}
$$

taking values in $[-\pi, \pi][3]$. Above, " $g$ " and " $a$ " are gauge coupling and lattice spacing parameters, respectively. The Euclidean action symmetrized between $\theta$ and $\theta^{\prime}$ variables 
for pure $\mathrm{U}(1)$ theory in temporal gauge on a lattice with $d$ spatial dimensions is given explicitly by $[15,16]$

$$
\begin{aligned}
S_{E}\left(n_{t}, n_{t}+1\right) \\
=-\frac{1}{2 g^{2}} \sum_{\boldsymbol{r}} \sum_{i \neq j=1}^{d}\left[2-\cos \left(\theta^{(\boldsymbol{r}, i)}+\theta^{(\boldsymbol{r}+\hat{i}, j)}-\theta^{(\boldsymbol{r}+\hat{j}, i)}-\theta^{(\boldsymbol{r}, j)}\right)\right. \\
\left.\quad-\cos \left(\theta^{\prime(\boldsymbol{r}, i)}+\theta^{\prime(\boldsymbol{r}+\hat{i}, j)}-\theta^{\prime(\boldsymbol{r}+\hat{j}, i)}-\theta^{\prime(\boldsymbol{r}, j)}\right)\right] \\
\quad-\frac{1}{g^{2}} \sum_{\boldsymbol{r}} \sum_{i=1}^{d}\left[1-\cos \left(\theta^{(\boldsymbol{r}, i)}-\theta^{\prime(\boldsymbol{r}, i)}\right)\right]
\end{aligned}
$$

with $\hat{i}$ as the unit vector along the spatial direction $i$. For a spatial lattice with $N_{\mathrm{L}}$ number of links and $N_{\mathrm{P}}$ number of plaquettes, it is convenient to define the plaquette-link matrix $\boldsymbol{M}$ of dimension $N_{\mathrm{P}} \times N_{\mathrm{L}}$, as following explicitly by its elements

$M_{l}^{p}= \begin{cases}+1, & \operatorname{link} l=(\boldsymbol{r}, i) \text { belongs to oriented plaquette } p \\ -1, & \text { link } l=(\boldsymbol{r},-i) \text { belongs to oriented plaquette } p . \\ 0, & \text { otherwise }\end{cases}$

In Fig. 2, the above definition is presented graphically. The explicit representations for $d=2$ case will be given later. The elements of the transfer-matrix $\hat{V}$ is defined in terms of the Euclidean action between two adjacent times

$$
\left\langle n_{t}+1|\hat{V}| n_{t}\right\rangle \propto e^{S_{E}\left(n_{t}, n_{t}+1\right)} .
$$

Setting

$$
\gamma=\frac{1}{g^{2}}
$$

and labeling links as $l=(\boldsymbol{r}, i)$ and plaquettes as " $p$," in terms of matrix $\boldsymbol{M}$ the elements of the transfer matrix $\hat{V}$ are given in the field basis by

$$
\begin{aligned}
\left\langle\boldsymbol{\theta}^{\prime}|\hat{V}| \boldsymbol{\theta}\right\rangle= & \mathcal{A} \prod_{p} \exp \left\{-\frac{\gamma}{2}\left[2-\cos \left(M_{l}^{p} \theta^{l}\right)-\cos \left(M_{l}^{p} \theta^{\prime l}\right)\right]\right\} \\
& \times \prod_{l} \exp \left\{-\gamma\left[1-\cos \left(\theta^{l}-\theta^{\prime l}\right)\right]\right\}
\end{aligned}
$$

in which the summations over repeated indices are understood. Above, $\mathcal{A}$ is inserted to fix the normalization [17].

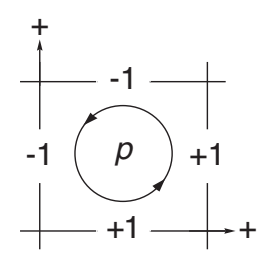

FIG. 2. Graphical representation of definition (3).
The aim is to formulate the theory in the field Fourier basis $\left|k_{l}\right\rangle$, which is related to the compact $\theta$ basis by

$\left\langle\theta^{l^{\prime}} \mid k_{l}\right\rangle=\frac{\delta_{l}^{l^{\prime}}}{\sqrt{2 \pi}} \exp \left(\mathrm{i} k_{l} \theta^{l}\right), \quad k_{l}=0, \pm 1, \pm 2, \cdots$.

Using an identity that involves the modified Bessel function $\mathrm{I}_{n}(x)$

$$
\exp (x \cos \phi)=\sum_{n} \mathrm{I}_{n}(x) \exp (\mathrm{i} n \phi)
$$

and the relation

$$
\int_{-\pi}^{\pi} \mathrm{d} \theta \exp (\mathrm{i} n \theta)=2 \pi \delta(n),
$$

one directly finds the matrix elements of $\hat{V}$ in the field Fourier basis [14]

$$
\begin{aligned}
\left\langle\boldsymbol{k}^{\prime}|\hat{V}| \boldsymbol{k}\right\rangle= & \mathcal{A} e^{-\gamma\left(N_{\mathrm{P}}+N_{\mathrm{L}}\right)}(2 \pi)^{N_{\mathrm{L}}} \sum_{\left\{n_{p}\right\}} \sum_{\left\{n_{p}^{\prime}\right\}} \prod_{p} \mathrm{I}_{n_{p}}\left(\frac{\gamma}{2}\right) \mathrm{I}_{n_{p}^{\prime}}\left(\frac{\gamma}{2}\right) \\
& \times \prod_{l} \mathrm{I}_{m_{l}}(\gamma) \delta\left[\left(n_{p}+n_{p}^{\prime}\right) M_{l}^{p}+k_{l}-k_{l}^{\prime}\right],
\end{aligned}
$$

in which $m_{l}=k_{l}+\sum_{p} n_{p} M_{l}^{p}=k_{l}^{\prime}-\sum_{p} n_{p}^{\prime} M_{l}^{p}$. Above $n_{p}, n_{p}^{\prime}$, and $m_{l}$ are all integer valued. The general solutions of the $\delta \mathrm{s}$ in the summations are given by means of the vectors satisfying [14]

$$
n^{0} \cdot M=\mathbf{0} .
$$

These vectors include $\boldsymbol{n}^{\mathbf{0}}=\mathbf{0}$. The general solution of the delta is then given as

$$
n_{p}+n_{p}^{\prime}=Q_{p}+n_{p}^{0}
$$

with $Q_{p}$ s being integer valued. The above solution leads to the following condition to have a nonzero matrix element (10) in the Fourier basis

$$
\boldsymbol{k}^{\prime}=\boldsymbol{k}+\boldsymbol{Q} \cdot \boldsymbol{M}
$$

Later the physical meaning of the above condition, by means of the current conservation between initial and final states at adjacent times, will be given. Accordingly, it is shown that $\hat{V}$ in the Fourier basis is block diagonal [14], and all elements of a block can be represented by a Fourier vector $\boldsymbol{k}_{*}$, whose coblocks are all constructed as

$$
\boldsymbol{k}_{* \boldsymbol{q}}=\boldsymbol{k}_{*}+\boldsymbol{q} \cdot \boldsymbol{M},
$$

in which $\boldsymbol{q}$ is a vector with $N_{\mathrm{P}}$ integers as components. We will see that the condition that two coblocks differ as above 
is a manifestation of the current conservation, allowing us to have a nonzero matrix element. By setting $\boldsymbol{Q}=\boldsymbol{q}^{\prime}-\boldsymbol{q}$, we have two coblock vectors

$$
\boldsymbol{k}_{* q}=\boldsymbol{k}_{*}+\boldsymbol{q} \cdot \boldsymbol{M}, \quad \boldsymbol{k}_{* q^{\prime}}^{\prime}=\boldsymbol{k}_{*}+\boldsymbol{q}^{\prime} \cdot \boldsymbol{M}
$$

for which the matrix element, using $\mathrm{I}_{m}=\mathrm{I}_{-m}$ and $n_{p}+q_{p} \rightarrow n_{p}$, is given by [14]

$$
\begin{aligned}
\left\langle\boldsymbol{k}_{* q^{\prime}}^{\prime}|\hat{V}| \boldsymbol{k}_{* \boldsymbol{q}}\right\rangle_{\boldsymbol{k}_{*}}= & \mathcal{A} e^{-\gamma\left(N_{\mathrm{P}}+N_{\mathrm{L}}\right)}(2 \pi)^{N_{\mathrm{L}}} \\
& \times \sum_{\left\{n_{p}^{0}\right\}} \sum_{\left\{n_{p}\right\}} \prod_{p} \mathrm{I}_{q_{p}-n_{p}}\left(\frac{\gamma}{2}\right) \mathrm{I}_{q_{p}^{\prime}-n_{p}+n_{p}^{0}}\left(\frac{\gamma}{2}\right) \\
& \times \prod_{l} \mathrm{I}_{k_{*}+\sum_{p} n_{p} M_{l}^{p}}(\gamma)
\end{aligned}
$$

in which no constraint on the summations is present, except that $n_{p}^{0}$ s satisfy (11). The important fact is that the allowed $n_{p}^{0}$ s are not dependent on $\boldsymbol{k}_{*}, \boldsymbol{q}$, and $\boldsymbol{q}^{\prime}$, but only on the matrix $\boldsymbol{M}$.

As the final point in this part, since there are infinite possible choices for $\boldsymbol{q s}$, each block is in fact infinite dimensional. One of the most special blocks is the one represented by the vacuum state $\boldsymbol{k}_{*}=\boldsymbol{k}_{\mathbf{0}}=\mathbf{0}$. In Ref. [14], it is shown that, provided that the ground state is unique, it belongs to the vacuum block. The reason is simply that, in the extreme large coupling limit $g \rightarrow \infty(\gamma \rightarrow 0)$, as all elements except $V_{00}$ are approaching zero, the energy and $\hat{V}$ eigenvalues are related,

$$
\varepsilon_{i}=-\frac{1}{a} \ln v_{i},
$$

and the ground state belongs to the vacuum's block. By uniqueness of the ground state, upon lowering the coupling, no crossing between the ground state by other states occurs, leaving the ground states in the vacuum block at any coupling [14].

\section{CURRENTS, STATES AND BLOCKS}

In this section, the basic elements and notions that emerged in the derivation of the previous chapter are discussed. In particular, the role of $k_{l}$ and $q_{p}$ numbers, the meaning of conditions (11) and (13), as well as some graphical representations are presented. To cover the basic idea, the detailed presentation is for the case of 2D spatial lattice, although the general expectations and differences of 3D case are discussed as well.

The first step is to understand the physical interpretation of the Fourier vectors $\boldsymbol{k}$. That is most directly understood by the way that these vectors appear, namely by Eqs. (1) and (7) and their similar expressions in the continuum theory, for the coupling of the current $J$ to the gauge field $A$ :

$$
e^{\mathrm{i} \sum_{l} k_{l} \theta^{l}}=e^{\mathrm{i} a g \sum_{l} k_{l} A^{l}} \rightarrow e^{\mathrm{i} g \int J \cdot A d x} .
$$

Above, $k_{l}$ is interpreted as the number of current quanta at link $l$, coupled to the gauge field $A^{l}$ associated with this link. So the current vector $\boldsymbol{k}$ consists of link currents $k_{l}$ s. The integer nature of $k_{l}$ reflects the fact that, due to the compact nature of gauge fields in the lattice formulation, the quantization of charge is satisfied automatically.

By the above interpretation of $k_{l} \mathrm{~s}$, the Fourier basis $|\boldsymbol{k}\rangle$ is representing the state with a set of current quanta $k_{l}$ on link $l$, with $l \in$ links. Now by the definition of the transfer matrix $\hat{V}=\exp (-a \hat{H})$, with the Hamiltonian $\hat{H}$, the matrix element $\left\langle\boldsymbol{k}^{\prime}|\hat{V}| \boldsymbol{k}\right\rangle$ is the transition amplitude between states with $\boldsymbol{k}$ and $\boldsymbol{k}^{\prime}$ currents during the imaginary time interval " $a . "$

Before proceeding, it is useful to find an explicit representation for the plaquette-link matrix $\boldsymbol{M}$. For the 2D periodic lattice with $N_{s}$ sites in each direction, there are $N_{\mathrm{P}}=N_{s}^{2}$ plaquettes and $N_{\mathrm{L}}=2 N_{s}^{2}$ links. For the plaquette and link numbering of the $3 \times 3$ periodic lattice given in Fig. 3, using the definition (3), one finds the following form for the $9 \times 18$ dimensional matrix $\boldsymbol{M}$ [14]

$$
\boldsymbol{M}=\left(\begin{array}{cccccccccccccccccc}
+ & - & 0 & 0 & 0 & 0 & 0 & 0 & 0 & - & 0 & 0 & + & 0 & 0 & 0 & 0 & 0 \\
0 & + & - & 0 & 0 & 0 & 0 & 0 & 0 & 0 & - & 0 & 0 & + & 0 & 0 & 0 & 0 \\
- & 0 & + & 0 & 0 & 0 & 0 & 0 & 0 & 0 & 0 & - & 0 & 0 & + & 0 & 0 & 0 \\
0 & 0 & 0 & + & - & 0 & 0 & 0 & 0 & 0 & 0 & 0 & - & 0 & 0 & + & 0 & 0 \\
0 & 0 & 0 & 0 & + & - & 0 & 0 & 0 & 0 & 0 & 0 & 0 & - & 0 & 0 & + & 0 \\
0 & 0 & 0 & - & 0 & + & 0 & 0 & 0 & 0 & 0 & 0 & 0 & 0 & - & 0 & 0 & + \\
0 & 0 & 0 & 0 & 0 & 0 & + & - & 0 & + & 0 & 0 & 0 & 0 & 0 & - & 0 & 0 \\
0 & 0 & 0 & 0 & 0 & 0 & 0 & + & - & 0 & + & 0 & 0 & 0 & 0 & 0 & - & 0 \\
0 & 0 & 0 & 0 & 0 & 0 & - & 0 & + & 0 & 0 & + & 0 & 0 & 0 & 0 & 0 & -
\end{array}\right) .
$$




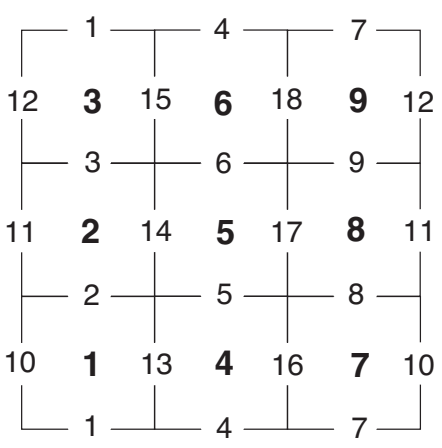

FIG. 3. The numbering of links and plaquettes for $3 \times 32 \mathrm{D}$ periodic lattice used in Eq. (19) as the representation of matrix $\boldsymbol{M}$ [14].

In the 2D lattice, each link is being shared by two plaquettes, resulting in only one "+" and one "-" in each column. The general form of the matrix $\boldsymbol{M}$ can be given by means of the $N_{s} \times N_{s}$ translation matrix $\boldsymbol{T}$ as follows [14]:

$$
\boldsymbol{M}=\left(\begin{array}{l|l}
\mathbb{1}_{N_{s}} \otimes \boldsymbol{T} & -\boldsymbol{T} \otimes \mathbb{1}_{N_{s}}
\end{array} .\right.
$$

By construction, the matrix $\boldsymbol{M}$ is $N_{s}^{2} \times 2 N_{s}^{2}$ dimensional, as it should. The elements of $\boldsymbol{T}$ are given by Ref. [14]

$$
T_{a b}=\delta_{a b}-\delta_{a+1, b}-\delta_{a, N_{s}} \delta_{b 1}, \quad a, b=1, \ldots, N_{s} .
$$

By the explicit form of the matrix $\boldsymbol{M}$, the meaning of the condition (13) to have a nonzero matrix element can be understood by means of the current conservation, as follows. Let us begin with the vacuum state $\boldsymbol{k}_{\mathbf{0}}=\mathbf{0}$, which, according to (18), represents the state with no current on any links. Now, by Eq. (13) and using

$$
\boldsymbol{q}_{\mathbf{1}}=\underbrace{(1,0, \ldots, 0)}_{N_{\mathrm{P}}},
$$

a coblock of the vacuum state is found as the following current vector

$$
k_{0 ; 1}=k_{0}+q_{1} \cdot M,
$$

in which only four links of the first plaquette have nonzero unit currents, namely two $+1 \mathrm{~s}$ and two $-1 \mathrm{~s}$, making a circulating unit current in the first plaquette, as represented in Fig. 4. Then the nonzero matrix element $\left\langle\boldsymbol{k}_{\mathbf{0}}|\hat{V}| \boldsymbol{k}_{\mathbf{0} ; \mathbf{1}}\right\rangle$ is made possible since two states differ in a unit current circulating in a plaquette, as required by current conservation.

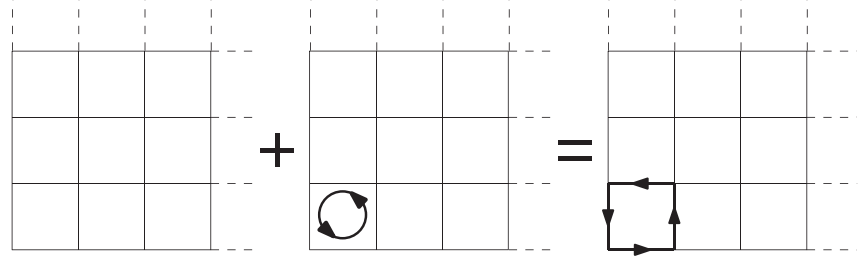

FIG. 4. The graphical representation of (23) to construct $\boldsymbol{k}_{\mathbf{0} ; \mathbf{1}}$ as a coblock of $\boldsymbol{k}_{\mathbf{0}}=\mathbf{0}$.

As seen in the above example, $q_{p}$ is determining the number of current quanta circulating in the plaquette $p$. It is befitted to call the $q_{p}$ numbers as plaquette currents or loop currents. As another example from the vacuum block, consider the state constructed by all $q_{p}$ s zero, except two of the nonadjacent plaquettes, as depicted in Fig. 5.

Let us go beyond the vacuum block by considering the state represented by the vector current

$$
\boldsymbol{k}_{\mathbf{1}}=\underbrace{(1,0, \ldots, 0)}_{N_{\mathrm{L}}},
$$

which has one unit of current on the first link of the lattice, with all other link currents zero. There is no set of plaquette currents that could yield this vector from the vacuum. Equivalently, one can say that there is no $\boldsymbol{q} \cdot \boldsymbol{M}$ equal to (24). So the state (24) does not belong to the vacuum block. Two coblocks of this state are presented in Figs. 6 and 7, one with the loop current in the first plaquette and one nonadjacent to the first plaquette, respectively. It is easy to check that there is a plaquette current that relates the resulting states in Figs. 6 and 7 as well. Again as the differences of these three current states are just loop currents circulating in plaquettes, the transition between

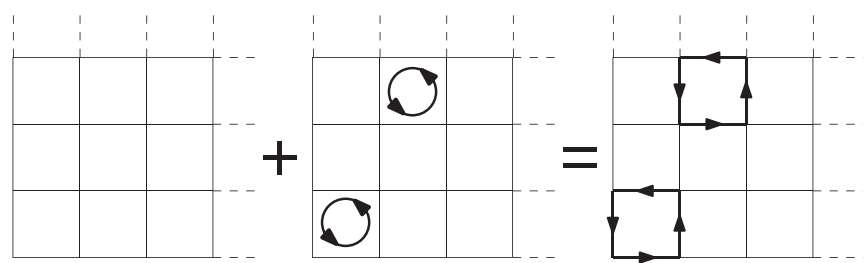

FIG. 5. Construction of a coblock of $\boldsymbol{k}_{\mathbf{0}}$ with two nonadjacent plaquette currents.

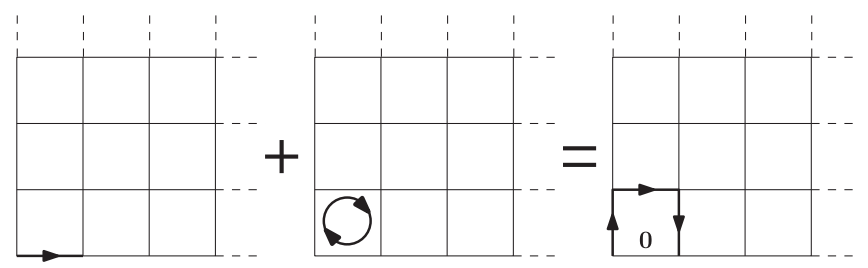

FIG. 6. $\boldsymbol{k}_{\mathbf{1}-\mathbf{1}}=\boldsymbol{k}_{\mathbf{1}}-\boldsymbol{q}_{\mathbf{1}} \cdot \boldsymbol{M}$ as a coblock of $\boldsymbol{k}_{\mathbf{1}}$ with three links having a unit current. 


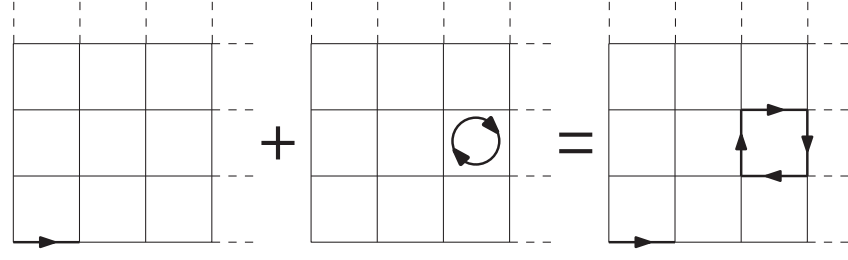

FIG. 7. A coblock of $\boldsymbol{k}_{\mathbf{1}}$ with five links having a unit current.

the two of them is possible, leading to the nonzero $\hat{V}$ elements between them.

As another example, consider the state represented by the vector current below

$$
\boldsymbol{k}_{\mathbf{2}}=\underbrace{(2,0, \ldots, 0)}_{N_{\mathrm{L}}}
$$

which has two units of current on the first link, with all others zero. Again it is easy to check that this state does not belong to the vacuum and $\boldsymbol{k}_{1}$ blocks. A coblock of this state is presented in Fig. 8. The difference between the direction of currents in the resulting state here with the last one in Fig. 4 is to be noted.

The last example, which will be used later, is the first vector in Fig. 9,

$$
\boldsymbol{k}_{\mathbf{1}, \mathbf{- 1}}=\underbrace{(1,-1,0, \ldots, 0)}_{N_{\mathrm{L}}},
$$

and its coblock on the right-hand side. The two vectors both have one unit of current on only two links, and in this respect are equivalent to be selected as representative of the block.

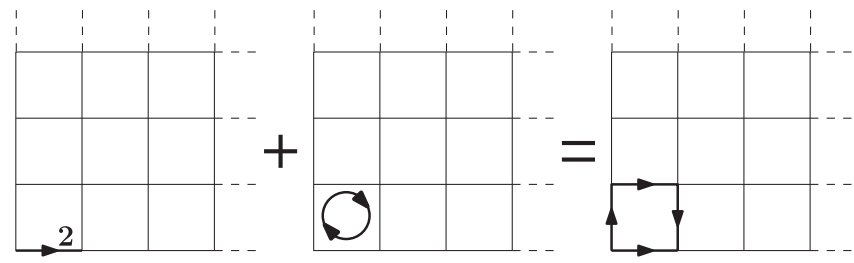

FIG. 8. $\boldsymbol{k}_{\mathbf{2} ;-\mathbf{1}}=\boldsymbol{k}_{\mathbf{2}}-\boldsymbol{q}_{\mathbf{1}} \cdot \boldsymbol{M}$ as a coblock of $\boldsymbol{k}_{\mathbf{2}}$ with four links at the first plaquette having unit current.

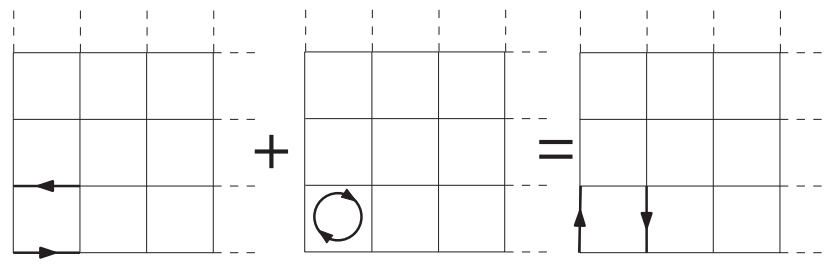

FIG. 9. $\boldsymbol{k}_{1,-1 ;-1}=\boldsymbol{k}_{1,-1}-\boldsymbol{q}_{\mathbf{1}} \cdot \boldsymbol{M}$ as a coblock of $\boldsymbol{k}_{\mathbf{1},-1}$, both with two links having a unit current.
By the representation of matrix $\boldsymbol{M}$ it is easy to check that for the periodic 2D lattices, the subspace by vectors $\boldsymbol{n}^{\mathbf{0}}$ satisfying (11) is one dimensional with the general form

$$
\boldsymbol{n}^{\mathbf{0}}=n^{0} \underbrace{(1,1, \ldots, 1)}_{N_{\mathrm{P}}}
$$

leaving all link currents zero by equal $n^{0}$ assigned to all plaquettes [14]. The condition (11) and its solution (27) may be represented by the graphical representation we used in different examples for $\boldsymbol{q} \cdot \boldsymbol{M}$ as depicted in Fig. 10. The loop-current vectors $\boldsymbol{n}^{\mathbf{0}}$ are specially important to manage the orders of Bessels in Eq. (16), and more related to this work, when the strong coupling expansion in $\gamma$ is being developed. We postpone the second for later when the expansion in $\gamma$ will be discussed. About the Bessels, for the 2D lattices, ranks of all Bessels $\mathrm{I}_{q_{p}^{\prime}-n_{p}-n^{0}}$ in Eq. (16) are affected by $n^{0} \neq 0$ equally. For definiteness, by setting $\boldsymbol{k}_{*}=\boldsymbol{q}=\boldsymbol{q}^{\prime}=\mathbf{0}$ in Eq. (16), let us consider the vacuum-tovacuum (VTV) element $\langle\mathbf{0}|\hat{V}| \mathbf{0}\rangle$ for the 2D lattice case:

$$
\begin{aligned}
\left\langle\mathbf{0}\left|\hat{V}_{2 \mathrm{~d}}\right| \mathbf{0}\right\rangle_{\mathbf{0}}= & \mathcal{A} e^{-\gamma\left(N_{\mathrm{P}}+N_{\mathrm{L}}\right)}(2 \pi)^{N_{\mathrm{L}}} \\
& \times \sum_{n^{0}} \sum_{\left\{n_{p}\right\}} \prod_{p} \mathrm{I}_{-n_{p}}\left(\frac{\gamma}{2}\right) \mathrm{I}_{-n_{p}-n^{0}}\left(\frac{\gamma}{2}\right) \\
& \times \prod_{l} \mathrm{I}_{\sum_{p} n_{p} M_{l}^{p}}(\gamma),
\end{aligned}
$$

in which by Eq. (27) the index " $p$ " is dropped from $n_{p}^{0}$. The formal expansion on Bessel functions in the strong coupling $\gamma=1 / g^{2} \ll 1$ is mainly based on the ordering relation between the Bessel's as $\mathrm{I}_{n}(x)>\mathrm{I}_{m}(x)$ for $|n|<|m|$, coming in the strong form when $x \ll 1$

$$
\mathrm{I}_{0}(x) \gg \mathrm{I}_{ \pm 1}(x) \gg \mathrm{I}_{ \pm 2}(x) \gg \mathrm{I}_{ \pm 3}(x) \gg \cdots
$$

For example, let us compare two terms in Eq. (28) with all $n_{p}$ s equal to zero, but in one $n^{0}=0$ and in the other $n^{0}=1$,

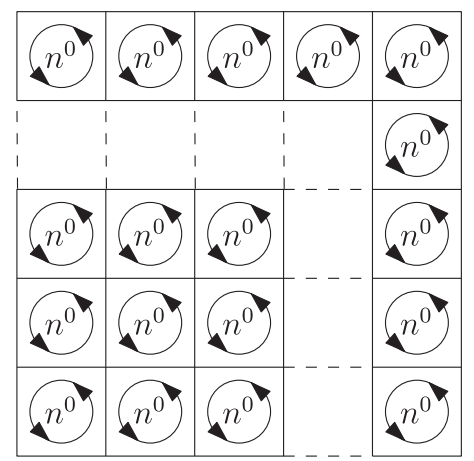

FIG. 10. Equally $n^{0}$ current units in all plaquettes make the net current at all links zero, provided that opposite edges are being identified in the periodic 2D lattice. 


$$
\begin{aligned}
\left\langle\mathbf{0}\left|\hat{V}_{2 \mathrm{~d}}\right| \mathbf{0}\right\rangle_{\mathbf{0}} & =\mathcal{A} e^{-\gamma\left(N_{\mathrm{P}}+N_{\mathrm{L}}\right)}(2 \pi)^{N_{\mathrm{L}}}\left[\mathrm{I}_{0}^{2 N_{\mathrm{P}}}\left(\frac{\gamma}{2}\right) \mathrm{I}_{0}^{N_{\mathrm{L}}}(\gamma)+\mathrm{I}_{0}^{N_{\mathrm{P}}}\left(\frac{\gamma}{2}\right) \mathrm{I}_{1}^{N_{\mathrm{P}}}\left(\frac{\gamma}{2}\right) \mathrm{I}_{0}^{N_{\mathrm{L}}}(\gamma)\right]+\cdots, \\
& =\mathcal{A} e^{-\gamma\left(N_{\mathrm{P}}+N_{\mathrm{L}}\right)}(2 \pi)^{N_{\mathrm{L}}} \mathrm{I}_{0}^{2 N_{\mathrm{P}}}\left(\frac{\gamma}{2}\right) \mathrm{I}_{0}^{N_{\mathrm{L}}}(\gamma)\left[1+\left(\frac{\mathrm{I}_{1}(\gamma / 2)}{\mathrm{I}_{0}(\gamma / 2)}\right)^{N_{\mathrm{P}}}\right]+\cdots
\end{aligned}
$$

Now, for large lattice size $N_{\mathrm{P}} \gg 1$ the term with the power of $N_{\mathrm{P}}$ can be dropped safely in comparison to one. In fact, a simple inspection shows that, depending on $\boldsymbol{q}$ or $\boldsymbol{q}^{\prime}$ defining an element, there are one or two choices for $n^{0}$ in which the Bessels of lowest order appear, and the other values of $n^{0}$ for large lattices can be ignored in the summation. The 2D latices may be contrasted to the 3D lattices, in which a finite number of nonzero loop currents may generate a vector $\boldsymbol{n}^{\mathbf{0}}$ satisfying $\boldsymbol{n}^{\mathbf{0}} \cdot \boldsymbol{M}=\mathbf{0}$. One case with the lowest number of nonzero loop currents is presented in Fig. 11, in which six plaquettes making a cube and their currents are presented. By the right-hand rule, all currents in Fig. 11 point outward the cube. The only other case with the lowest number is that with all loop currents reversed, pointing inward the cube. As all link currents by the configuration are zero, the loop currents presented in Fig. 11 satisfy $\boldsymbol{n}^{\mathbf{0}} \cdot \boldsymbol{M}=\mathbf{0}$. As a consequence, the term $\mathrm{I}_{1} / \mathrm{I}_{0}$ in Eq. (30) for a $3 \mathrm{D}$ lattice would have the power of 6 instead of $N_{\mathrm{P}}$. Any number of such cubes when sitting together also make a $\boldsymbol{n}^{\mathbf{0}}$ vector that satisfies the condition (11).

\section{CURRENT EXPANSION AT LARGE COUPLING}

The aim in this section is to provide the basic rules of the strong coupling expansion in $\gamma=1 / g^{2} \ll 1$ for the elements of the transfer-matrix in the field Fourier basis. In the next section and Appendix C, several examples of the application of rules for the strong coupling expansion are given. The samples of expansion in $\gamma$ presented in this work can be checked either by massive direct integration over field space or by the expression (16), provided that the Bessels with relevant ranks are identified.

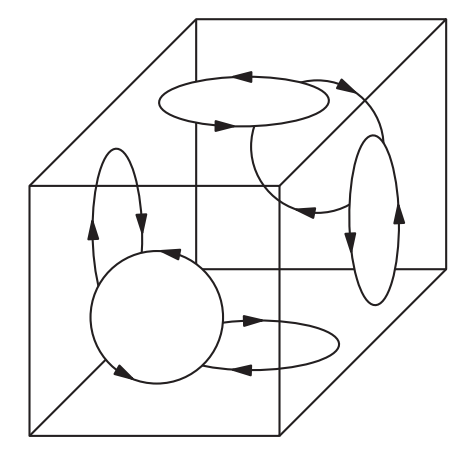

FIG. 11. The six-plaquette cube configuration in a 3D lattice that satisfies $\boldsymbol{n}^{\mathbf{0}} \cdot \boldsymbol{M}=\mathbf{0}$. All currents point outward by right-hand rule.
In the previous section, the role of integer numbers $k_{l}$, $q_{p}$, and $n_{p}^{0}$ together with their graphical representations are introduced. Based on the notions developed before, it will be shown that the strong coupling expansion can be regarded as the summations on currents. In particular, a nonzero element of the transfer matrix, as the transition amplitude between two current states belonging to the same block, is interpreted as the summations on occurrences of virtual loop and link currents, each weighted by $\gamma$, that transform both states to the vacuum.

The presentation is coming in two subsections. First, by working out some examples of the matrix elements at lower orders, it is seen how the expansion based on loop and link currents would emerge. In the second subsection, the set of rules of current expansion in the strong coupling regime is presented.

\section{A. Basic observations on current expansion}

To begin with, it is convenient to define the reduced form of the transfer matrix as

$$
\hat{V}=\mathcal{A} e^{-\gamma\left(N_{\mathrm{L}}+N_{\mathrm{P}}\right)}(2 \pi)^{N_{\mathrm{L}}} \underline{\hat{V}},
$$

for which the extra numerical factors are dropped, with the element in field basis as

$$
\begin{aligned}
\left\langle\boldsymbol{\theta}^{\prime}|\hat{\underline{V}}| \boldsymbol{\theta}\right\rangle= & \frac{1}{(2 \pi)^{N_{\mathrm{L}}}} \prod_{p} \exp \left\{\frac{\gamma}{2}\left[\cos \left(M_{l}^{p} \theta^{l}\right)+\cos \left(M_{l}^{p} \theta^{\prime l}\right)\right]\right\} \\
& \times \prod_{l} \exp \left\{\gamma \cos \left(\theta^{l}-\theta^{\prime l}\right)\right\} .
\end{aligned}
$$

The element of the reduced form in the Fourier basis is given by

$$
\left\langle\boldsymbol{k}^{\prime}|\hat{\underline{V}}| \boldsymbol{k}\right\rangle_{\boldsymbol{k}_{*}}=\frac{1}{(2 \pi)^{N_{\mathrm{L}}}} \int_{-\pi}^{\pi} \prod_{l} \mathrm{~d} \theta_{l}^{\prime} \mathrm{d} \theta_{l} e^{-\mathrm{i} \boldsymbol{k}^{\prime} \cdot \boldsymbol{\theta}^{\prime}} e^{\mathrm{i} \boldsymbol{k} \cdot \boldsymbol{\theta}}\left\langle\boldsymbol{\theta}^{\prime}|\underline{\hat{V}}| \boldsymbol{\theta}\right\rangle,
$$

in which the representative vector of the block is $\boldsymbol{k}_{*}$, and the $\boldsymbol{k}$ and $\boldsymbol{k}^{\prime}$ vectors are given by means of loop currents $\boldsymbol{q}$ and $\boldsymbol{q}^{\prime}$ as (15). Let us begin with the matrix elements in the vacuum block by setting $\boldsymbol{k}_{*}=\mathbf{0}$, for which we have

$$
\begin{aligned}
& \left\langle\boldsymbol{q}^{\prime} \cdot \boldsymbol{M}|\underline{\hat{V}}| \boldsymbol{q} \cdot \boldsymbol{M}\right\rangle_{\mathbf{0}} \\
& \quad=\frac{1}{(2 \pi)^{N_{\mathrm{L}}}} \int_{-\pi}^{\pi} \prod_{l} \mathrm{~d} \theta_{l}^{\prime} \mathrm{d} \theta_{l} e^{-\mathrm{i} \boldsymbol{q}^{\prime} \cdot \boldsymbol{M} \cdot \boldsymbol{\theta}^{\prime}} e^{\mathrm{i} \boldsymbol{q} \cdot \boldsymbol{M} \cdot \boldsymbol{\theta}}\left\langle\boldsymbol{\theta}^{\prime}|\underline{\hat{V}}| \boldsymbol{\theta}\right\rangle .
\end{aligned}
$$


At the first order in $\gamma$ it is easy to find the following terms:

$$
\begin{aligned}
\left\langle\boldsymbol{q}^{\prime} \cdot \boldsymbol{M}|\hat{\underline{V}}| \boldsymbol{q} \cdot \boldsymbol{M}\right\rangle_{\mathbf{0}}= & \prod_{l} \delta\left(\left(\boldsymbol{q}^{\prime} \cdot \boldsymbol{M}\right)_{l}\right) \delta\left((\boldsymbol{q} \cdot \boldsymbol{M})_{l}\right) \\
& +\frac{\gamma}{4} \prod_{l} \delta\left((\boldsymbol{q} \cdot \boldsymbol{M})_{l}\right) \sum_{p^{\prime}}\left[\prod_{l^{\prime}} \delta\left(\left(\boldsymbol{q}^{\prime} \cdot \boldsymbol{M}\right)_{l^{\prime}}+M_{l^{\prime}}^{p^{\prime}}\right)+\prod_{l^{\prime}} \delta\left(\left(\boldsymbol{q}^{\prime} \cdot \boldsymbol{M}\right)_{l^{\prime}}-M_{l^{\prime}}^{p^{\prime}}\right)\right] \\
& +\frac{\gamma}{4} \prod_{l^{\prime}} \delta\left(\left(\boldsymbol{q}^{\prime} \cdot \boldsymbol{M}\right)_{l^{\prime}}\right) \sum_{p}\left[\prod_{l} \delta\left((\boldsymbol{q} \cdot \boldsymbol{M})_{l}+M_{l}^{p}\right)+\prod_{l} \delta\left((\boldsymbol{q} \cdot \boldsymbol{M})_{l}-M_{l}^{p}\right)\right] \\
& +\frac{\gamma}{2} \sum_{l_{1}}\left[\prod_{l} \delta\left((\boldsymbol{q} \cdot \boldsymbol{M})_{l}+\delta_{l l_{1}}\right) \delta\left(\left(\boldsymbol{q}^{\prime} \cdot \boldsymbol{M}\right)_{l}+\delta_{l l_{1}}\right)\right. \\
& \left.+\prod_{l} \delta\left((\boldsymbol{q} \cdot \boldsymbol{M})_{l}-\delta_{l l_{1}}\right) \delta\left(\left(\boldsymbol{q}^{\prime} \cdot \boldsymbol{M}\right)_{l}-\delta_{l l_{1}}\right)\right]+\mathrm{O}\left(\gamma^{2}\right),
\end{aligned}
$$

in which $(\boldsymbol{q} \cdot \boldsymbol{M})_{l}$ is the current on link $l$. It is noted that, in the last term, the link index $l_{1}$ is common between two $\delta \mathrm{s}$ by $\boldsymbol{q}^{\prime}$ and $\boldsymbol{q}$, as originated from the term $\cos \left(\theta-\theta^{\prime}\right)$, in which $\theta$ and $\theta^{\prime}$ are appearing equally. The other point about the last term is this: in the vacuum block and for any plaquette vectors $\boldsymbol{q}$ and $\boldsymbol{q}^{\prime}$, there is no chance that all multiplying $\delta$ s would be satisfied, leading to zero value for this term. This is simply because there are always nonzero arguments for some of the $\delta \mathrm{s}$, and so this term has no contribution. The vacuum to vacuum (VTV) transition with $\boldsymbol{q}=\boldsymbol{q}^{\prime}=\mathbf{0}$, by $\delta(m)=\delta(-m)$ takes the form

$$
\langle\mathbf{0}|\underline{\hat{V}}| \mathbf{0}\rangle_{\mathbf{0}}=1+\gamma \sum_{p} \prod_{l} \delta\left(M_{l}^{p}\right)+\mathrm{O}\left(\gamma^{2}\right) .
$$

Due to the nonzero elements of $M_{l}^{p}$ at each plaquette, the linear term in $\gamma$ vanishes, leading to

$$
\langle\mathbf{0}|\underline{\hat{V}}| \mathbf{0}\rangle_{\mathbf{0}}=1+\mathrm{O}\left(\gamma^{2}\right) .
$$

At this order now, let us consider the element by vacuum and the current vector (23), by setting $\boldsymbol{q}^{\prime}=\boldsymbol{q}_{\mathbf{1}}$ and $\boldsymbol{q}=\mathbf{0}$ with one unit of loop current in the first plaquette as in Fig. 4. By Eq. (35) then the transition between vacuum and $\left|\boldsymbol{k}_{\mathbf{0} ; \mathbf{1}}\right\rangle=|\mathbf{1}\rangle$ is given by

$$
\begin{aligned}
\langle\mathbf{1}|\hat{\underline{V}}| \mathbf{0}\rangle_{\mathbf{0}}= & \prod_{l} \delta\left(M_{l}^{1}\right)+\frac{\gamma}{2} \sum_{p^{\prime}} \prod_{l^{\prime}} \delta\left(M_{l^{\prime}}^{p^{\prime}}\right) \prod_{l} \delta\left(M_{l}^{1}\right) \\
& +\frac{\gamma}{4} \sum_{p^{\prime}}\left[\prod_{l^{\prime}} \delta\left(M_{l^{\prime}}^{1}+M_{l^{\prime}}^{p^{\prime}}\right)+\prod_{l^{\prime}} \delta\left(M_{l^{\prime}}^{1}-M_{l^{\prime}}^{p^{\prime}}\right)\right] \\
& +\mathrm{O}\left(\gamma^{2}\right)
\end{aligned}
$$

in which the first and second terms do not contribute, due to the nonzero elements by $M_{l^{\prime}}^{1}$ or $M_{l^{\prime}}^{p^{\prime}}$. The third term, however, contributes due to the term $\delta\left(M_{l^{\prime}}^{1}-M_{l^{\prime}}^{p^{\prime}}\right)$ for $p^{\prime}=1$ in the summation, leading to

$$
\langle\mathbf{1}|\underline{\hat{V}}| \mathbf{0}\rangle_{\mathbf{0}}=\frac{\gamma}{4}+\mathrm{O}\left(\gamma^{2}\right)
$$

As a graphical representation for the cancellation between link currents and loop current in the same plaquette in the $\delta$, one may consider the diagram in Fig. 12 for the transition $\mathbf{1} \rightarrow \mathbf{0}$.

In fact, Fig. 12 is similar to Fig. 4, except that the loop current is plotted in a reversed and dashed form in the $\boldsymbol{k}_{\mathbf{0} ; \mathbf{1}}$ side. The reason for being dashed is to emphasize its virtual nature. As a space-saving representation, one may suggest the following for Fig. 12

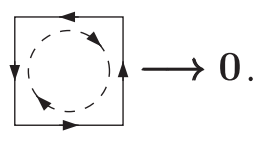

It can be seen that, at the first order of $\gamma$, the only nonzero elements are the third term of (38), like Fig. 12 or (40), with one loop current to vacuum. In the vacuum block, the order of $\gamma^{2}$ can be obtained by the expansion of the exponential as well. To present the long expressions in a compact form from now on, the alternative representations are used as

$$
\begin{aligned}
\boldsymbol{q} \cdot \boldsymbol{M} & \rightarrow \boldsymbol{q}, & \boldsymbol{q}^{\prime} \cdot \boldsymbol{M} & \rightarrow \boldsymbol{q}^{\prime}, \\
(\boldsymbol{q} \cdot \boldsymbol{M})_{l} & \rightarrow q_{l}, & \left(\boldsymbol{q}^{\prime} \cdot \boldsymbol{M}\right)_{l} & \rightarrow q_{l}^{\prime},
\end{aligned}
$$

in which at order $\gamma^{2}$ in the vacuum block, we have

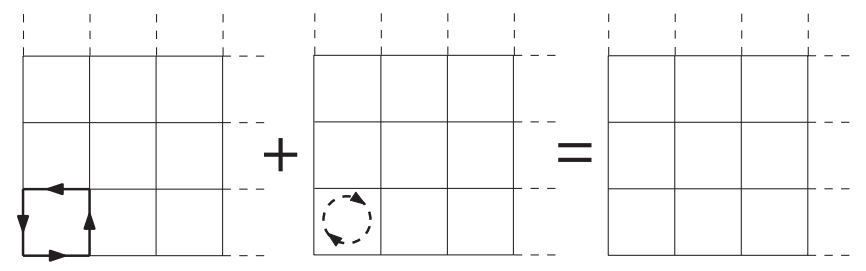

FIG. 12. The combination of $\boldsymbol{k}_{\mathbf{0} ; \mathbf{1}}$ and virtual loop current. 


$$
\begin{aligned}
& {\left[\left\langle\boldsymbol{q}^{\prime}|\underline{\hat{V}}| \boldsymbol{q}\right\rangle_{\mathbf{0}}\right]_{\gamma^{2}}=\frac{\gamma^{2}}{32} \prod_{l} \delta\left(\not q_{l}\right) \sum_{p_{1}^{\prime}, p_{2}^{\prime}}\left[\prod_{l^{\prime}} \delta\left(\not_{l^{\prime}}^{\prime}+M_{l^{\prime}}^{p_{1}^{\prime}}+M_{l^{\prime}}^{p_{2}^{\prime}}\right)+2 \prod_{l^{\prime}} \delta\left(\not \phi_{l^{\prime}}^{\prime}+M_{l^{\prime}}^{p_{1}^{\prime}}-M_{l^{\prime}}^{p_{2}^{\prime}}\right)+\prod_{l^{\prime}} \delta\left(\not \phi_{l^{\prime}}^{\prime}-M_{l^{\prime}}^{p_{1}^{\prime}}-M_{l^{\prime}}^{p_{2}^{\prime}}\right)\right]} \\
& +\frac{\gamma^{2}}{32} \prod_{l^{\prime}} \delta\left(\not l_{l^{\prime}}^{\prime}\right) \sum_{p_{1}, p_{2}}\left[\prod_{l} \delta\left(\not \phi_{l}+M_{l}^{p_{1}}+M_{l}^{p_{2}}\right)+2 \prod_{l} \delta\left(\not q_{l}+M_{l}^{p_{1}}-M_{l}^{p_{2}}\right)+\prod_{l} \delta\left(\not \phi_{l}-M_{l}^{p_{1}}-M_{l}^{p_{2}}\right)\right] \\
& +\frac{\gamma^{2}}{16} \sum_{p, p^{\prime}}\left[\prod_{l} \delta\left(\not q_{l}+M_{l}^{p}\right) \delta\left(\not q_{l}^{\prime}-M_{l}^{p^{\prime}}\right)+\prod_{l} \delta\left(\not q_{l}+M_{l}^{p}\right) \delta\left(\not \phi_{l}^{\prime}+M_{l}^{p^{\prime}}\right)\right. \\
& \left.+\prod_{l} \delta\left(\not q_{l}-M_{l}^{p}\right) \delta\left(\not l_{l}^{\prime}-M_{l}^{p^{\prime}}\right)+\prod_{l} \delta\left(\not q_{l}-M_{l}^{p}\right) \delta\left(\not l_{l}^{\prime}+M_{l}^{p^{\prime}}\right)\right] \\
& +\frac{\gamma^{2}}{8} \sum_{l_{1}, l_{2}}\left[\prod_{l} \delta\left(\not l_{l}+\delta_{l l_{1}}+\delta_{l l_{2}}\right) \delta\left(\not l_{l}^{\prime}+\delta_{l l_{1}}+\delta_{l l_{2}}\right)+\prod_{l} \delta\left(\not l_{l}+\delta_{l l_{1}}-\delta_{l l_{2}}\right) \delta\left(\not l_{l}^{\prime}+\delta_{l l_{1}}-\delta_{l l_{2}}\right)\right. \\
& \left.+\prod_{l} \delta\left(\not l_{l}-\delta_{l l_{1}}+\delta_{l l_{2}}\right) \delta\left(\not q_{l}^{\prime}-\delta_{l l_{1}}+\delta_{l l_{2}}\right)+\prod_{l} \delta\left(\not l_{l}-\delta_{l l_{1}}-\delta_{l l_{2}}\right) \delta\left(\not q_{l}^{\prime}-\delta_{l l_{1}}-\delta_{l l_{2}}\right)\right] \\
& +\frac{\gamma^{2}}{8} \sum_{p^{\prime}, l}\left[\prod_{l^{\prime}} \delta\left(\not l_{l^{\prime}}^{\prime}-M_{l^{\prime}}^{p^{\prime}}+\delta_{l l^{\prime}}\right) \delta\left(\not l_{l^{\prime}}+\delta_{l l^{\prime}}\right)+\prod_{l^{\prime}} \delta\left(\phi_{l^{\prime}}^{\prime}+M_{l^{\prime}}^{p^{\prime}}+\delta_{l l^{\prime}}\right) \delta\left(\not l l^{\prime}+\delta_{l l^{\prime}}\right)\right] \\
& +\frac{\gamma^{2}}{8} \sum_{p^{\prime}, l}\left[\prod_{l^{\prime}} \delta\left(\not l_{l^{\prime}}^{\prime}-M_{l^{\prime}}^{p^{\prime}}-\delta_{l l^{\prime}}\right) \delta\left(\not l_{l^{\prime}}-\delta_{l l^{\prime}}\right)+\prod_{l^{\prime}} \delta\left(\not l_{l^{\prime}}^{\prime}+M_{l^{\prime}}^{p^{\prime}}-\delta_{l l^{\prime}}\right) \delta\left(\not l_{l^{\prime}}-\delta_{l l^{\prime}}\right)\right] \\
& +\frac{\gamma^{2}}{8} \sum_{p, l^{\prime}}\left[\prod_{l} \delta\left(\not q_{l}-M_{l}^{p}+\delta_{l l^{\prime}}\right) \delta\left(\not q_{l}^{\prime}+\delta_{l l^{\prime}}\right)+\prod_{l} \delta\left(\not q_{l}+M_{l}^{p}+\delta_{l l^{\prime}}\right) \delta\left(\not l_{l}^{\prime}+\delta_{l l^{\prime}}\right)\right] \\
& +\frac{\gamma^{2}}{8} \sum_{p, l^{\prime}}\left[\prod_{l} \delta\left(\not q_{l}-M_{l}^{p}-\delta_{l l^{\prime}}\right) \delta\left(\not l_{l}^{\prime}-\delta_{l l^{\prime}}\right)+\prod_{l} \delta\left(\not q_{l}^{\prime}+M_{l}^{p^{\prime}}-\delta_{l l^{\prime}}\right) \delta\left(\not q_{l}-\delta_{l l^{\prime}}\right)\right] \text {. }
\end{aligned}
$$

Again the last four sums, containing the combination of one $\cos \left(M_{l}^{p} \theta^{l}\right)$ or $\cos \left(M_{l}^{p} \theta^{\prime l}\right)$ with one $\cos \left(\theta-\theta^{\prime}\right)$, has $\delta \mathrm{s}$ with common link indices $l$ or $l^{\prime}$. Also, in the vacuum block, there is no chance that these terms would survive, as it is not possible that all multiplying $\delta$ s would be satisfied simultaneously. Let us consider the $\gamma^{2}$ contribution for the VTV transition with $\boldsymbol{q}=\boldsymbol{q}^{\prime}=\mathbf{0}$

$$
\begin{aligned}
{\left[\langle\mathbf{0}|\hat{V}| \mathbf{0}\rangle_{\mathbf{0}}\right]_{\gamma^{2}}=} & \frac{\gamma^{2}}{8} \sum_{p_{1}, p_{2}}\left[\prod_{l} \delta\left(M_{l}^{p_{1}}+M_{l}^{p_{2}}\right)+\prod_{l} \delta\left(M_{l}^{p_{1}}-M_{l}^{p_{2}}\right)\right]+\frac{\gamma^{2}}{4} \sum_{p, p^{\prime}} \prod_{l} \delta\left(M_{l}^{p}\right) \delta\left(M_{l}^{p^{\prime}}\right) \\
& +\frac{\gamma^{2}}{4} \sum_{l_{1}, l_{2}}\left[\prod_{l} \delta\left(\delta_{l l_{1}}+\delta_{l l_{2}}\right) \delta\left(\delta_{l l_{1}}+\delta_{l l_{2}}\right)+\prod_{l} \delta\left(\delta_{l l_{1}}-\delta_{l l_{2}}\right) \delta\left(\delta_{l l_{1}}-\delta_{l l_{2}}\right)\right] .
\end{aligned}
$$

In the first term, only the second part may survive by cancellation between $M$ s, provided that $p_{1}$ and $p_{2}$ refer to the same plaquette, developing a factor of $N_{\mathrm{P}}$ by the summation over all plaquettes. The second term vanishes, as for each plaquette there are four links for which $M_{l}^{p}$ is nonzero. In the last term, again, the second part may contribute by cancellation between $\delta_{l l_{i}}$ if $l_{1}$ and $l_{2}$ refer to the same link, giving a factor of $N_{\mathrm{L}}$. With these, we have

$$
\left[\langle\mathbf{0}|\underline{\hat{V}}| \mathbf{0}\rangle_{\mathbf{0}}\right]_{\gamma^{2}}=\frac{\gamma^{2}}{8} N_{\mathrm{P}}+\frac{\gamma^{2}}{4} N_{\mathrm{L}} .
$$

The above may be represented graphically as follows: 


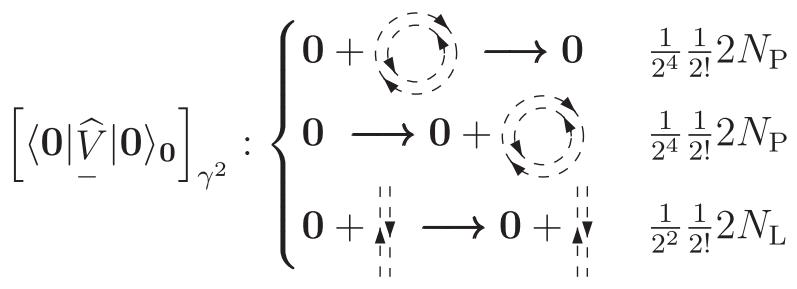

The rules for associated numerical factors and the powers of $\gamma$ will be discussed later. Equation (37) combined with (44) leads to

$$
\langle\mathbf{0}|\underline{\hat{V}}| \mathbf{0}\rangle_{\mathbf{0}}=1+\left(\frac{N_{\mathrm{P}}}{8}+\frac{N_{\mathrm{L}}}{4}\right) \gamma^{2}+\mathrm{O}\left(\gamma^{3}\right)
$$

For the $\mathbf{1} \rightarrow \mathbf{0}$ transition by setting $\boldsymbol{q}^{\prime}=\boldsymbol{q}_{\mathbf{1}}$ and $\boldsymbol{q}=\mathbf{0}$, it is easy to see that always unsatisfied $\delta$ s would remain, showing that this transition will not take contribution at order $\gamma^{2}$. Now consider the $\gamma^{2}$ contribution for the $\mathbf{1} \rightarrow \mathbf{1}$, for which the only surviving term is

$$
\left[\langle\mathbf{1}|\underline{\hat{V}}| \mathbf{1}\rangle_{\mathbf{0}}\right]_{\gamma^{2}}=\frac{\gamma^{2}}{16} \sum_{p, p^{\prime}}\left[\prod_{l} \delta\left(M_{l}^{1}-M_{l}^{p}\right) \delta\left(M_{l}^{1}-M_{l}^{p^{\prime}}\right)\right] .
$$

In the summation, the only contribution comes from $p=p^{\prime}=1$, by which $M_{l}^{p}$ and $M_{l}^{p^{\prime}}$ are canceled by $M_{l}^{1}$ in both $\delta$ s, leading to

$$
\langle\mathbf{1}|\underline{\hat{V}}| \mathbf{1}\rangle_{\mathbf{0}}=\frac{\gamma^{2}}{16}+\mathrm{O}\left(\gamma^{3}\right) .
$$

The naïve way of thinking about $\mathbf{1} \rightarrow \mathbf{1}$ may suggest that it would always be possible as both initial and final states are the same. However, as seen in the extreme large coupling limit $\gamma \rightarrow 0$, this transition vanishes as $\gamma^{2}$. The expression obtained after doing the Fourier integrals shows that the $\delta$ s related to initial and final states must be satisfied separately to obtain a nonzero result. In other words, the final expression should be interpreted as the result of the transform of both initial and final states to the vacuum. Accordingly, as the transforms $\mathbf{1} \rightarrow \mathbf{0}$ and $\mathbf{0} \rightarrow \mathbf{1}$ at lowest order, each with a loop current transform to vacuum, are both proportional to $\gamma$, the $\mathbf{1} \rightarrow \mathbf{1}$ transition should be of order $\gamma^{2}$. This crucial role of vacuum that, as if it is being passed in $\mathbf{1} \rightarrow \mathbf{1}$ transition due to the nature of Fourier integrals, is true for all other transitions, including $\mathbf{0} \rightarrow \mathbf{0}$ by Eq. (46) and even those in blocks other than the vacuum. To avoid misinterpretation, it is quite suitable to highlight this role by the vacuum by changing the notation slightly from $\mathbf{1} \rightarrow \mathbf{1}$ to $\mathbf{1} \rightarrow \mathbf{0} \rightarrow \mathbf{1}$, or simply $\stackrel{0}{\rightarrow} \mathbf{1}$. By this, the graphical representation for the above transition may be given as below

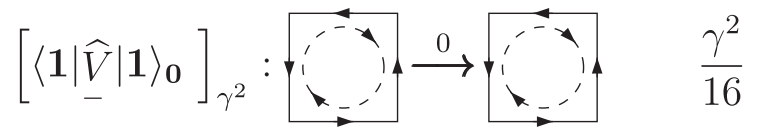

The order of $\gamma^{3}$ in the vacuum block is given in Appendix A with about 80 terms. It is obvious that the terms with one $\delta_{l l_{i}}$ or three $\delta_{l l_{i}}$ would not survive in the vacuum block, as there is no chance that all multiplying $\delta \mathrm{s}$ would be satisfied. So the surviving terms at third order in the vacuum block are those of the first three lines of (A2). For the VTV transition with $\boldsymbol{q}=\boldsymbol{q}^{\prime}=\mathbf{0}$, again we see that there is no way that all multiplying $\delta$ s would be satisfied. By $\boldsymbol{q}^{\prime}=\boldsymbol{q}_{\mathbf{1}}$ and $\boldsymbol{q}=\mathbf{0}$ with one unit of loop current in the first plaquette as in Fig. 4, when one or three $M \mathrm{~s}$ are in the $q_{1}$ side, there is the chance that all $\delta$ s would be satisfied. The only thing is to calculate the combinatorial factors for all possible states that would lead to satisfying all $\delta$ s. For the first term, the possible ways would be three combinations of two $-M$ s and one $+M$,

$$
q_{1},-M,-M,+M \rightarrow 0,
$$

in which $q_{1}$ cancels with one of $-M \mathrm{~s}$, and two others cancel out each other. Depending on whether all $M \mathrm{~s}$ are in the $q_{1}$ plaquette or not, we have the combinatorial factor

$$
3\left(2\left(N_{\mathrm{P}}-1\right)+1\right)=3\left(2 N_{\mathrm{P}}-1\right) .
$$

The second term may contribute by

$$
q_{1},-M \rightarrow 0,+M,-M
$$

having two possibilities for $+/-$ signs on the second side, giving the combinatorial factor

$$
2 N_{\mathrm{P}}
$$

The last term contribution comes from the combinations of

$$
q_{1},-M,+\delta,-\delta \rightarrow 0,+\delta,-\delta .
$$

Having in mind that the place of link $\delta \mathrm{s}$ in the lattice in two sides are the same, together with two possibilities for $+/-$ signs for $\delta$ s, leads to the combinatorial factor

$$
2 N_{\mathrm{L}} \text {. }
$$

It can be shown by the following graphical representation: 


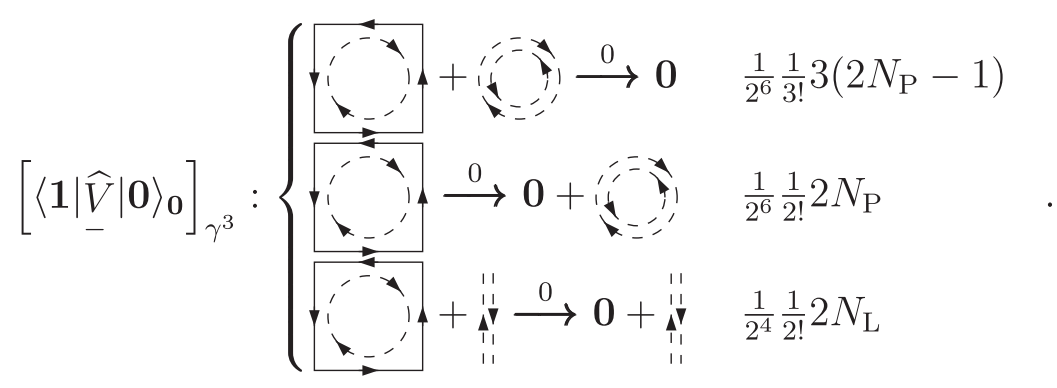

All together, using Eqs. (39) and (56), we have the result as follows:

$$
\langle\mathbf{1}|\underline{\hat{V}}| \mathbf{0}\rangle_{\mathbf{0}}=\frac{\gamma}{4}+\left(\frac{-1}{128}+\frac{N_{\mathrm{P}}}{32}+\frac{N_{\mathrm{L}}}{16}\right) \gamma^{3}+\cdots .
$$

Before proceeding to the rules in the next subsection, it is instructive to consider examples of the matrix elements in the nonvacuum block by setting the representative state $\boldsymbol{k}_{*} \neq \mathbf{0}$ in Eqs. (15) and (33). In particular, we consider the block with a representative state of (24) $\boldsymbol{k}_{*}=\boldsymbol{k}_{1}=$ $(1,0, \ldots, 0)$ from the previous section, with just one unit of current on the first link. It is easy to see that the zerothorder $\gamma^{0}$ belongs only to the VTV of the vacuum block, and so up to the first order of $\gamma$ in $\boldsymbol{k}_{\mathbf{1}}$ s block we find

$$
\begin{aligned}
\left\langle\boldsymbol{k}_{\mathbf{1}}+\boldsymbol{q}|\hat{\underline{V}}| \boldsymbol{k}_{\mathbf{1}}+\boldsymbol{q}\right\rangle_{\mathbf{1}}= & \frac{\gamma}{4} \prod_{l} \delta\left(k_{1 l}+\not \phi_{l}\right) \sum_{p^{\prime}}\left[\prod_{l^{\prime}} \delta\left(k_{1 l}+\not l_{l^{\prime}}^{\prime}+M_{l^{\prime}}^{p^{\prime}}\right)+\prod_{l^{\prime}} \delta\left(k_{1 l}+\not q_{l^{\prime}}^{\prime}-M_{l^{\prime}}^{p^{\prime}}\right)\right] \\
& +\frac{\gamma}{4} \prod_{l^{\prime}} \delta\left(k_{1 l}+\phi_{l^{\prime}}^{\prime}\right) \sum_{p}\left[\prod_{l} \delta\left(k_{1 l}+\not q_{l}+M_{l}^{p}\right)+\prod_{l} \delta\left(k_{1 l}+\phi_{l}-M_{l}^{p}\right)\right] \\
& +\frac{\gamma}{2} \sum_{l_{1}}\left[\prod_{l} \delta\left(k_{1 l}+\phi_{l}+\delta_{l l_{1}}\right) \delta\left(k_{1 l}+\not l_{l}^{\prime}+\delta_{l l_{1}}\right)+\prod_{l} \delta\left(k_{1 l}+\not q_{l}-\delta_{l l_{1}}\right) \delta\left(k_{1 l}+\not q_{l}^{\prime}-\delta_{l l_{1}}\right)\right] \\
& +\mathrm{O}\left(\gamma^{2}\right) .
\end{aligned}
$$

Using the fact that $\not$ and $\boldsymbol{q}$ ' add loop currents to the representative state $\boldsymbol{k}_{\mathbf{1}}$, it is shown that the first order in this block survives only for $\boldsymbol{q}=\boldsymbol{q}^{\prime}=\mathbf{0}$, leading to the matrix element

$$
\begin{aligned}
\left\langle\boldsymbol{k}_{1}|\underline{\hat{V}}| \boldsymbol{k}_{1}\right\rangle_{\mathbf{1}}= & \frac{\gamma}{2} \sum_{l_{1}}\left[\prod_{l} \delta\left(k_{1 l}-\delta_{l l_{1}}\right) \delta\left(k_{1 l}-\delta_{l l_{1}}\right)\right] \\
& +\mathrm{O}\left(\gamma^{2}\right)
\end{aligned}
$$

for which, setting $l_{1}=1$ by the $\delta$ s, we find

$$
\left\langle\boldsymbol{k}_{1}|\underline{\hat{V}}| \boldsymbol{k}_{1}\right\rangle_{\mathbf{1}}=\frac{\gamma}{2}+\mathrm{O}\left(\gamma^{2}\right)
$$

The expression (59) shows explicitly how the separate multiplying $\delta$ s connect the initial and final states to the vacuum state, although it does not belong to $\boldsymbol{k}_{\mathbf{1}}$ block. The crucial role of the vacuum state, as mentioned before, is related to the fact that both $\delta$ s by initial and final states are to be satisfied, as if both passed through the vacuum state. Accordingly, the graphical representation of the above is expressed as follows:

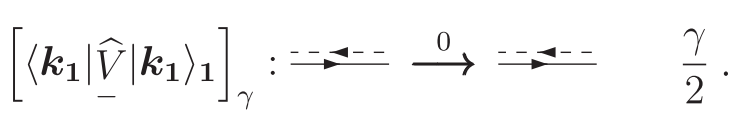

At the next order now let us consider the element by setting $\boldsymbol{q}^{\prime}=\mathbf{0}$ and $\boldsymbol{q}=\boldsymbol{q}_{\mathbf{1}}=(1,0, \ldots, 0)$ of Eq. (22). The current vector $\boldsymbol{k}_{1}+\not \phi_{1}=\boldsymbol{k}_{1 ; 1}$ with one unit of loop current and link current in the first plaquette, may be represented as

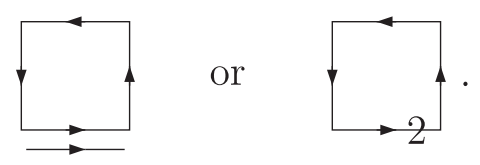

In the following, for the sake of clarity, we use the first of the above. Then the surviving matrix element is found to be

$$
\begin{aligned}
\left\langle\boldsymbol{k}_{\mathbf{1}}|\underline{\hat{V}}| \boldsymbol{k}_{\mathbf{1} ; \mathbf{1}}\right\rangle_{\mathbf{1}}= & \frac{\gamma^{2}}{8} \sum_{p_{1}} \sum_{l_{1}}\left[\prod_{l} \delta\left(k_{1 l}-\delta_{l l_{1}}\right)\right. \\
& \left.\times \delta\left(k_{1 l}+M_{l}^{1}-M_{l}^{p_{1}}-\delta_{l l_{1}}\right)\right]+\mathrm{O}\left(\gamma^{3}\right) .
\end{aligned}
$$

Above, the cancellation of link currents in both $\delta$ s and loop currents in the second $\delta$ by $p_{1}=1$ leads to 


$$
\left\langle\boldsymbol{k}_{1}|\underline{\hat{V}}| \boldsymbol{k}_{\mathbf{1} ; \mathbf{1}}\right\rangle_{\mathbf{1}}=\frac{\gamma^{2}}{8}+\mathrm{O}\left(\gamma^{3}\right)
$$

Once again, the $\delta \mathrm{s}$ in expression (63) by the initial and final states show explicitly how the connections between two states and the vacuum, which do not even belong to this block, determine the strength of transition. Graphically the way of satisfying $\delta$ s may be represented as

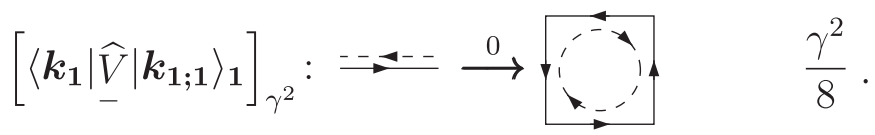

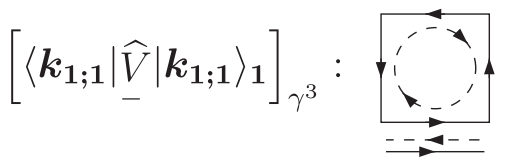

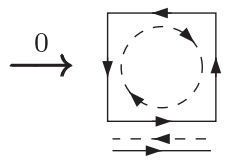

\section{B. Rules of current expansion in strong coupling}

As announced earlier, in the present subsection the set of rules is given in which at any order of $\gamma$ in principle, one can write the transfer-matrix element between two states. The rules are based on determining the ways that transform the initial and final states to the vacuum, accompanied by the associated numerical and combinatorial factors of each transition. As mentioned, the distinguished role of the vacuum state simply comes back to the fact that the $\delta \mathrm{s}$ related to the initial and final states' Fourier integrals are to be satisfied separately. Accordingly, and as seen in previous examples, the transform can be represented by a set of graphs in which a proper combination of virtual loop and link currents would make the required pass through the vacuum. Also, by the given examples, the transform to vacuum is to be considered even for states that do not belong to the vacuum block. This is because the coblocks of a state are determined by adding loop-currents, via $\boldsymbol{q} \cdot \boldsymbol{M}$ in Eq. (15), but the concerned transform to vacuum is due to both link and loop currents, the former via " $\cos \left(\theta-\theta^{\prime}\right)$ " term that is irrelevant for making coblocks. It is due to these link currents that transforming any state into a vacuum is made possible, even for those states in blocks other than the vacuum.

For any state in the Fourier basis, there are infinite ways to transform it into the vacuum. As seen before, this is correct for the vacuum state itself. For two given states of $|\boldsymbol{k}\rangle$ and $\left|\boldsymbol{k}^{\prime}\right\rangle$ in the same block, consider the case that they transform to vacuum by $m$ and $m^{\prime}$ numbers of virtual loop currents, respectively, accompanied by $\ell$ numbers of virtual link currents for both states. By Eq. (32) the mentioned numbers of currents appear through the integration
Let us consider the diagonal element by setting $\boldsymbol{q}^{\prime}=\boldsymbol{q}=\boldsymbol{q}_{1}$, with one unit of loop current and link current in the first plaquette, given by

$$
\begin{aligned}
\left\langle\boldsymbol{k}_{\mathbf{1 ; 1}}|\hat{\hat{V}}| \boldsymbol{k}_{\mathbf{1} ; \mathbf{1}}\right\rangle_{\mathbf{1}}= & \frac{\gamma^{3}}{32} \sum_{p, p^{\prime}, l_{1}} \prod_{l} \delta\left(k_{1 l}+M_{l}^{1}-M_{l}^{p^{\prime}}-\delta_{l l_{1}}\right) \\
& \times \delta\left(k_{1 l}+M_{l}^{1}-M_{l}^{p}-\delta_{l l_{1}}\right)+\mathrm{O}\left(\gamma^{4}\right) .
\end{aligned}
$$

This is the only term for this order. Four link currents are canceled by loop currents if $p=p^{\prime}=1$, however, $\boldsymbol{k}_{\mathbf{1}}$ should be canceled by $\delta_{l l_{1}} \mathrm{~s}$ in both sides, leading to

$$
\left\langle\boldsymbol{k}_{\mathbf{1 ; 1} \mathbf{1}}|\hat{\hat{V}}| \boldsymbol{k}_{\mathbf{1 ; 1}}\right\rangle_{\mathbf{1}}=\frac{\gamma^{3}}{32}+\mathrm{O}\left(\gamma^{4}\right)
$$

with the graphical representation as follows:

of $m, m^{\prime}$, and $\ell$ numbers of $\cos \left(M_{l}^{p} \theta^{l}\right) \mathrm{s}, \cos \left(M_{l}^{p} \theta^{\prime l}\right) \mathrm{s}$, and $\cos \left(\theta^{l}-\theta^{\prime l}\right) \mathrm{s}$, respectively. Now, as it can be derived easily (see Appendix B), the numerical factor associated with the matrix element of transition through the considered transform is

$\left[\left\langle\boldsymbol{k}^{\prime}|\underline{\hat{V}}| \boldsymbol{k}\right\rangle\right]_{m, m^{\prime}, \ell}=\mathcal{K}_{m, m^{\prime}, \ell} \frac{1}{2^{2 m+2 m^{\prime}+\ell}} \frac{1}{m ! m^{\prime} ! \ell !} \gamma^{m+m^{\prime}+\ell}$,

in which $\mathcal{K}_{m, m^{\prime}, \ell}$ is the combinatorial factor representing the number of ways that loop and link currents can be combined, regarding the initial and final transforms to the vacuum.

In the previous subsection, graphical representations are suggested to each term contributing to the transition at the lowest orders of $\gamma$. In fact, and as we will see in several examples, these graphical representations can be used to determine and manage the channels that contribute to a transition at a given order. In this respect, these graphical representations can serve as the Feynman diagrams in perturbative quantum field theory. The elements being used in graphs are simply the currents, loop or link ones, being characterized by their real or virtual natures. Accordingly, the initial and final states, being determined only by link currents, are interpreted as real and presented by a combination of solid lines as

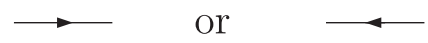

and their rotated versions. Instead, the loop and link currents that occurred during the transforms are interpreted as virtual. The virtual loop currents, representing the $\pm M_{l}^{p} \mathrm{~s}$ inside multiplying $\delta(\cdot) \mathrm{s}$, are coming as 


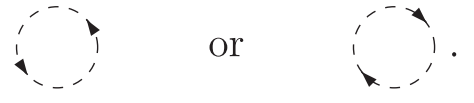

The virtual link currents, representing the $\delta_{l l^{\prime}} \mathrm{s}$ inside multiplying $\delta(\cdot) \mathrm{s}$, are drawn below and their rotated versions

$$
---\quad \text { or } \quad-\cdots-- \text {. }
$$

The important point about the virtual link currents is, as mentioned earlier, that they come in both sides of initial and final transforms to vacuum equally, since they are originated from Fourier integration over $\cos \left(\theta-\theta^{\prime}\right)$ s.

Some general statements about the expansion for an arbitrary element can be made and come in order. First, it can be easily shown that two subsequent orders of $\gamma$ in the expansion of an element differ by two. By the previous examples as well as several ones given later, that is evident. This simply comes back to the fact that any order for an element differs from a higher-order one by adding an even number of virtual currents to transform both initial and final states to vacuum. By this, the expansion for an element in $\boldsymbol{k}_{*}$ block looks like

$\left\langle\boldsymbol{k}_{* \boldsymbol{q}^{\prime}}^{\prime}|\underline{\hat{V}}| \boldsymbol{k}_{* \boldsymbol{q}}\right\rangle_{\boldsymbol{k}_{*}}=\gamma^{h}\left(c_{0}+c_{2} \gamma^{2}+c_{4} \gamma^{4}+c_{6} \gamma^{6}+\cdots\right)$,

in which $h$ is the lowest order at which the transforms of both initial and final states to vacuum, by adding virtual link and loop currents, are made possible. So by $\gamma=1 / g^{2}$, the subsequent increase of order is in fact $1 / g^{4}$, which makes the expansion fairly reliable for even not so large values of $g$. The value of $h$ can be determined as well, once $\boldsymbol{k}_{*}, \boldsymbol{q}$, and $\boldsymbol{q}^{\prime}$ are given. From Sec. III, we know that each vector current inside a block can be selected as the representative. To make things systematically, we use the convention that the representative vector would have the minimum value of

$$
|\boldsymbol{k}|=\sum_{l=1}^{N_{\mathrm{L}}}\left|k_{l}\right|
$$

In some blocks, the above specifies just one vector current. For example, in the vacuum block, it is only the vacuum and not, say, its coblock (23) in Fig. 4 with larger (74). The other block with $\boldsymbol{k}_{\mathbf{1}}$ in Eq. (24) and those in Figs. 6 and 7 as coblocks, again $\boldsymbol{k}_{1}$ has a minimum (74), is being taken as representative. The same is true for the block with $\boldsymbol{k}_{\mathbf{2}}$ and its coblock in Fig. 8. For some blocks, however, the condition would not identify just one candidate as the representative. An example is given in Fig. 9, in which both current vectors have equal (74), and so both can be taken as representatives. The least order of $h$ can be obtained simply by realizing how many link and loop currents are necessary to transform the given states to the vacuum. It is here that the representative $\boldsymbol{k}_{*}$ with a minimum (74) is needed. By $\boldsymbol{q}$ and $\boldsymbol{q}^{\prime}$ of (15) we then have

$$
h=\left|\boldsymbol{k}_{*}\right|+|\boldsymbol{q}|+\left|\boldsymbol{q}^{\prime}\right|,
$$

in which $\left|\boldsymbol{k}_{*}\right|$ as (74) and

$$
|\boldsymbol{q}|=\sum_{p=1}^{N_{\mathrm{P}}}\left|q_{p}\right|
$$

and a similar one for $\left|\boldsymbol{q}^{\prime}\right|$. The relation for " $h$ " is correct for all blocks, including those with more than one candidate as representative with a minimum (74), provided that $\boldsymbol{q}$ and $\boldsymbol{q}^{\prime}$ take those loop vectors, which satisfy (15) with the selected representative. The above for " $h$ " can be checked easily by the previous examples and several ones given later.

As the last feature, it is befitting to discuss here the effect of lattice dimension on the expansion. The first footstep of lattice dimension is seen by the number of links and plaquettes, $N_{\mathrm{L}}$ and $N_{\mathrm{P}}$, in the expansion. For example, for the 2D and 3D periodic spatial lattices with $N_{s}$ sites in each direction, we have the following

$$
\begin{array}{ll}
2 \mathrm{~d}: N_{\mathrm{L}}=2 N_{s}^{2}, & N_{\mathrm{P}}=N_{s}^{2}, \\
3 \mathrm{~d}: N_{\mathrm{L}}=3 N_{s}^{3}, & N_{\mathrm{P}}=3 N_{s}^{3} .
\end{array}
$$

The other impact of lattice dimension is related to the difference of null condition (11) for $\boldsymbol{n}^{\mathbf{0}}$ in different dimensions. In Sec. III for the 2D and 3D cases, the difference is mentioned, and its effect on managing Bessel orders in Eq. (16) is discussed. Here the effect of this difference in $\gamma$ expansion is pointed. The vector $\boldsymbol{n}^{\mathbf{0}}$ affects the expansion as it, via $\boldsymbol{n}^{\mathbf{0}} \cdot \boldsymbol{M}=\mathbf{0}$, can contribute to the transformations of states to vacuum. As seen in the $2 \mathrm{D}$ case, $\boldsymbol{n}^{\mathbf{0}}$ represents loop currents in all $N_{\mathrm{P}}$ plaquettes. So its presence affects the order $\gamma^{N_{\mathrm{P}}}$, which is quite suppressed for supposedly large lattices $N_{\mathrm{P}} \rightarrow \infty$ in the strong coupling limit $\gamma \ll 1$. In the $3 \mathrm{D}$ case, however, vector $\boldsymbol{n}^{\mathbf{0}}$ may represent a six-plaquette cubic configuration with net zero current, as shown in Fig. 11. Of course, there are many other finite-plaquette configurations with zero net currents, simply by combining the lower number ones. By these, we conclude that in form (73) the orders up to $\gamma^{4+h}$ are formally the same in all dimensions, however, the number of links and plaquettes should be replaced according to dimensions as in Eqs. (77) and (78). The first difference due to the configurations that do not fit in the 2D appears at order $6+h$. As an example, in the next section, the orders $\gamma^{4}$ and $\gamma^{6}$ of transition $\mathbf{0} \stackrel{0}{\rightarrow} \mathbf{0}$ are considered, which consist of the ways that combinations of virtual currents can take place in 2D. The $\gamma^{4}$ order result is formally the same in both dimensions, but for the $\gamma^{6}$ order, one has to take into account also the cube configuration in Fig. 11. Depending on whether the virtual 
currents on cube configuration are on the first or second side, we have for numbers in Eq. (69) the following possibilities

$$
\begin{array}{ll}
\boldsymbol{k}^{\prime} \text { side }: m=0, & m^{\prime}=6, \quad \ell=0, \\
\boldsymbol{k} \text { side }: m=6, & m^{\prime}=0, \quad \ell=0 .
\end{array}
$$

The evaluation of the combinatorial factor is given in the next section. In general, as the difference between 2D and 3D comes in the fourth term in the expansion of (73), namely in " $c_{6} \gamma^{6+h}$," the difference between the third and fourth terms is of order $\gamma^{2}=1 / g^{4}$, which for many practical purposes is not significant even for not so large coupling $g$.

\section{APPLICATION OF RULES}

The following examples are presented to see how the expression (69) and the associated graphical representation work in practice. The matrix-elements expansions up to order $\gamma^{3}$ are already presented, together with the associated graphical representations. Similar to order $\gamma^{3}$ in the vacuum block, the $\delta$ expansion of order $\gamma^{4}$ is given in Appendix A. Again in the vacuum block the terms with an odd number of $\delta_{l l_{i}}$ s have no chance to survive, specifically those in the last three lines in (A3). The VTV transition with $\boldsymbol{q}=\boldsymbol{q}^{\prime}=\mathbf{0}$, also terms with an odd number of $M \mathrm{~s}$ at $q$ and $q^{\prime}$ sides, have no chance [those in lines four and five of (A3)]. So only the terms in lines one to three contribute to VTV transition, for which the counting of combinatorial factors are given. For the first term, the possible ways would be six combinations of two $-M \mathrm{~s}$ and two $+M$, in which one $+M$ cancels out one of $-M \mathrm{~s}$, and two others cancel out each other. Depending on whether all $M \mathrm{~s}$ are in the same plaquette or not

$\left.\begin{array}{l}0 \rightarrow 0,-M,-M,+M,+M \\ 0,-M,-M,+M,+M \rightarrow 0\end{array}\right\}: C_{4}^{2}\left(2 N_{\mathrm{P}}\left(N_{\mathrm{P}}-1\right)+N_{\mathrm{P}}\right)$

in which

$$
C_{n}^{m}=\left(\begin{array}{c}
n \\
m
\end{array}\right)=\frac{n !}{m !(n-m) !} .
$$

The possible ways for the second term would be four combinations of two $M \mathrm{~s}$ and two $\delta$ s, in which $+M$ cancels with one of $-M \mathrm{~s}$, and $\delta$ s cancel out each other in two sides. So we have the combinatorial factor as

$$
\left.\begin{array}{l}
0,-M,+M,+\delta,-\delta \rightarrow 0,+\delta,-\delta \\
0,+\delta,-\delta \rightarrow 0,-M,+M,+\delta,-\delta
\end{array}\right\}: C_{2}^{1} C_{2}^{1} N_{\mathrm{P}} N_{\mathrm{L}}
$$

The third term may contribute as

$$
0,+M,-M \rightarrow 0,+M,-M: C_{2}^{1} C_{2}^{1} N_{\mathrm{P}} N_{\mathrm{P}}
$$

And the last combination at this order is

$$
0,+\delta,+\delta,-\delta,-\delta \rightarrow 0,+\delta,+\delta,-\delta,-\delta: C_{4}^{2}\left(2 N_{\mathrm{L}}\left(N_{\mathrm{L}}-1\right)+N_{\mathrm{L}}\right),
$$

which comes by considering whether all $\delta \mathrm{s}$ are in the same link or not. All of these can be expressed in the graphical representation below:

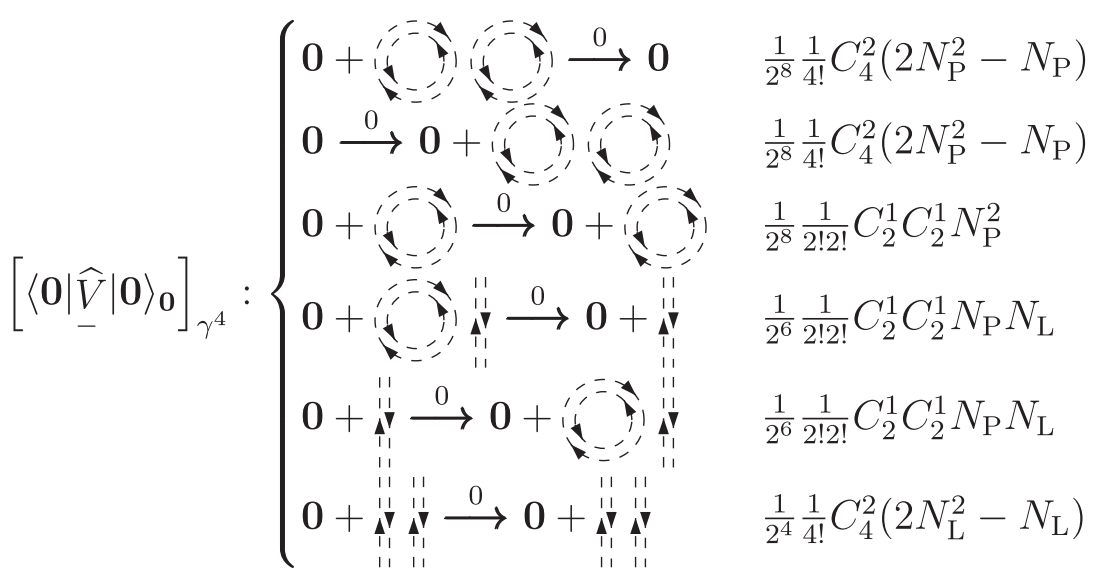

Using Eq. (46) results in 


$$
\begin{aligned}
\langle\mathbf{0}|\underline{\hat{V}}| \mathbf{0}\rangle_{\mathbf{0}}= & 1+\left(\frac{N_{\mathrm{P}}}{8}+\frac{N_{\mathrm{L}}}{4}\right) \gamma^{2} \\
& +\left(-\frac{N_{\mathrm{L}}}{64}+\frac{N_{\mathrm{L}}^{2}}{32}-\frac{N_{\mathrm{P}}}{512}+\frac{N_{\mathrm{P}} N_{\mathrm{L}}}{32}+\frac{N_{\mathrm{P}}^{2}}{128}\right) \gamma^{4} \\
& +\cdots
\end{aligned}
$$

It is easy to check that at order $\gamma^{4}$ the transition $\mathbf{1} \stackrel{0}{\rightarrow} \mathbf{0}$ does not find any contribution. Instead, the transition $\langle\mathbf{1}|\underline{\hat{V}}| \mathbf{1}\rangle_{\mathbf{0}}$ gets contributions at this order by the combination of loop and link currents as before. However, at this order, a new combination for cancellation between the initial real loop current and four virtual link currents takes place, graphically represented in Eq. (88).

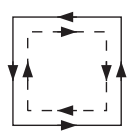

Let us start with already known combinations. First, we have the possibility that one $-M$ on one side and $+M,-M,-M$ on the other side would result in full cancellation toward vacuum. The combinatorial factor is then

$$
C_{3}^{1}\left(2 N_{\mathrm{P}}-1\right)
$$

The other possibility is one $-M$ and two $\pm \delta_{l l^{\prime}}$ s in each side, by the factor

$$
C_{2}^{1} N_{\mathrm{L}}
$$

The last is the new one (88), for which counting the ways that four $\pm \delta_{l l^{\prime}} \mathrm{s}$ take place gives the factor

$$
C_{4}^{2} 4
$$

These all are summarized below

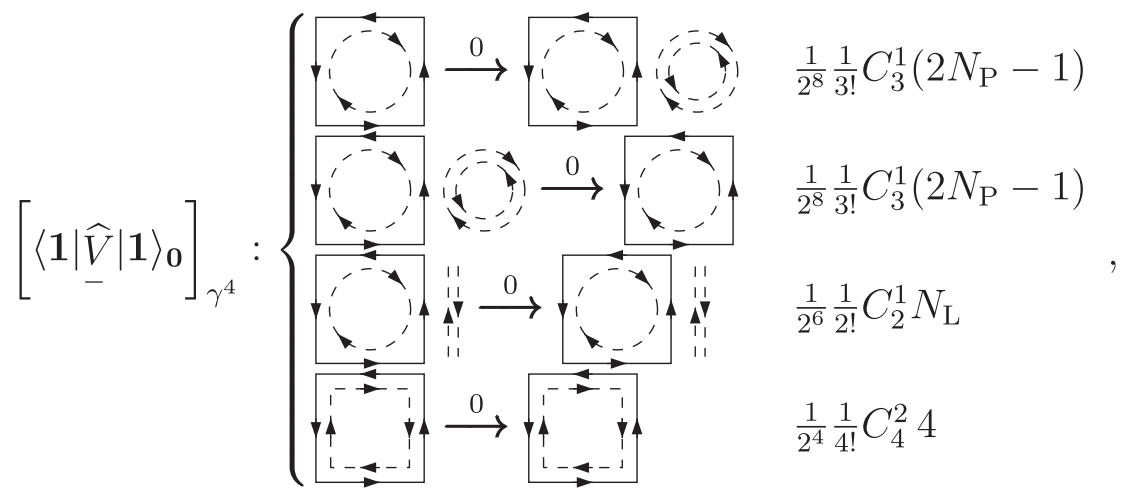

combined with the result of order $\gamma^{2}$ with this order giving

$$
\langle\mathbf{1}|\underline{\hat{V}}| \mathbf{1}\rangle_{\mathbf{0}}=\frac{\gamma^{2}}{16}+\left(\frac{15}{256}+\frac{N_{\mathrm{L}}}{64}+\frac{N_{\mathrm{P}}}{128}\right) \gamma^{4}+\cdots .
$$

At fifth order, it is easy to see that VTV does not get a contribution. Instead, the $\mathbf{1} \stackrel{0}{\rightarrow} \mathbf{0}$ transition takes the contribution from combinations of the initial loop-current $q_{1}$ and virtual loop and link currents as seen in previous examples. However, apart from Eq. (40), a new combination takes place; that is, four link currents may cancel out a virtual loop-current as shown in Eq. (94).

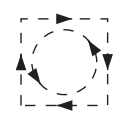

Again we start with more familiar cases. The first possibility is

$$
q_{1},-M,-M,-M,+M,+M \rightarrow 0,
$$

in which $q_{1}$ cancels with one of $-M \mathrm{~s}$, and four others cancel out each other. Depending on how many $M$ s sitting in the $q_{1}$ plaquette or are separated, we have the combinatorial factor

$$
\begin{aligned}
& C_{5}^{3}\left(3\left(2\left(N_{\mathrm{P}}-1\right)\left(N_{\mathrm{P}}-2\right)+N_{\mathrm{P}}-1\right)+3 \times 2\left(N_{\mathrm{P}}-1\right)+1\right) \\
& \quad=C_{5}^{3}\left(6 N_{\mathrm{P}}^{2}-9 N_{\mathrm{P}}+4\right) .
\end{aligned}
$$

The second may contribute as

$$
q_{1},-M,-M,+M \rightarrow 0,+M,-M
$$

having possibilities for $+/-$ signs in each of two sides, giving the combinatorial factor

$$
C_{2}^{1} C_{3}^{2} N_{\mathrm{P}}\left(2\left(N_{\mathrm{P}}-1\right)+1\right)=C_{2}^{1} C_{3}^{2} N_{\mathrm{P}}\left(2 N_{\mathrm{P}}-1\right)
$$

The third combination is

$$
q_{1},-M \rightarrow 0,+M,+M,-M,-M
$$


as $q_{1}$ cancels $-M$, and on the other side, four virtual currents cancel each other, with the combinatorial factor

$$
C_{4}^{2}\left(2 N_{\mathrm{P}}\left(N_{\mathrm{P}}-1\right)+N_{\mathrm{P}}\right)=C_{4}^{2}\left(2 N_{\mathrm{P}}^{2}-N_{\mathrm{P}}\right) .
$$

By

$$
q_{1},-M,-M,+M,+\delta,-\delta \rightarrow 0,+\delta,-\delta
$$

again $q_{1}$ is canceled by $-M$, and for two other $M \mathrm{~s}$ depending on sitting in the $q_{1}$ plaquette or not, we have the combinatorial factor

$$
C_{3}^{2} C_{2}^{1} N_{\mathrm{L}}\left(2\left(N_{\mathrm{P}}-1\right)+1\right)=C_{3}^{2} C_{2}^{1} N_{\mathrm{L}}\left(2 N_{\mathrm{P}}-1\right) .
$$

The fifth term at this order is by the combination

$$
q_{1},-M,+\delta,-\delta \rightarrow 0,+M,-M,+\delta,-\delta .
$$

In the second side, two $M$ s cancel out each other, and the $\delta$ s each other too, with the factor

$$
C_{2}^{1} C_{2}^{1} N_{\mathrm{L}} N_{\mathrm{P}}
$$

By the combination

$$
q_{1},-M,+\delta,+\delta,-\delta,-\delta \rightarrow 0,+\delta,+\delta,-\delta,-\delta
$$

the ways for cancellation of the four $\delta$ s give the combinatorial factor

$$
C_{4}^{2}\left(2 N_{\mathrm{L}}\left(N_{\mathrm{L}}-1\right)+N_{\mathrm{L}}\right)=C_{4}^{2}\left(2 N_{\mathrm{L}}^{2}-N_{\mathrm{L}}\right)
$$

In the last one, the four currents by $q_{1}$ are canceled with the four $\delta \mathrm{s}$

$$
q_{1},+\delta,+\delta,-\delta,-\delta \rightarrow 0,+M,+\delta,+\delta,-\delta,-\delta
$$

Having possibilities for $+/-$ with a fixed place for the $\delta \mathrm{s}$ in the first plaquette the number of combinations is

$$
C_{4}^{2} 2 \times 2
$$

The combinations associated with the transition are summarized below:

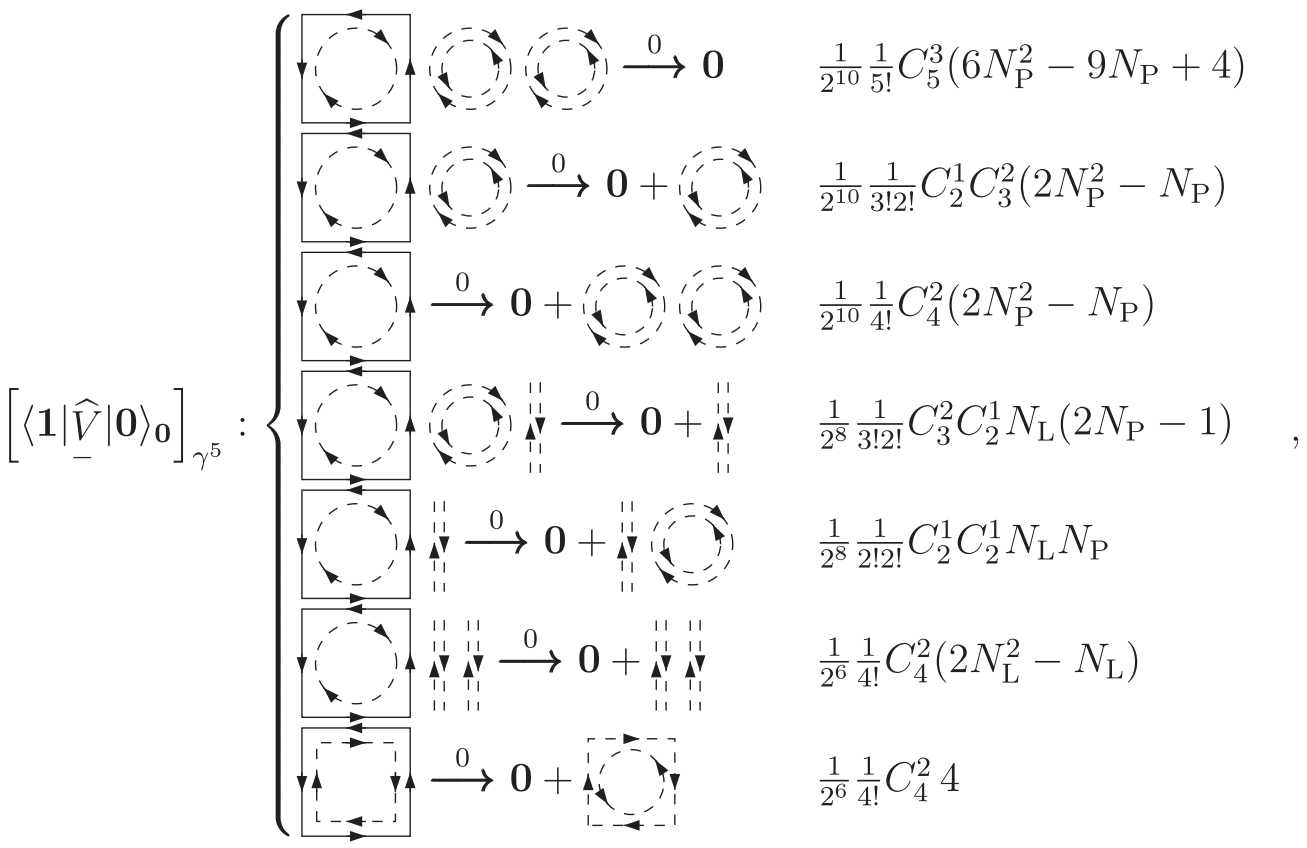

all together up to fifth order

$$
\begin{aligned}
\langle\mathbf{1}|\underline{\hat{V}}| \mathbf{0}\rangle_{\mathbf{0}}= & \frac{\gamma}{4}+\left(\frac{-1}{128}+\frac{N_{\mathrm{P}}}{32}+\frac{N_{\mathrm{L}}}{16}\right) \gamma^{3} \\
& +\left(\frac{49}{3072}-\frac{3 N_{\mathrm{L}}}{512}+\frac{N_{\mathrm{L}}^{2}}{128}-\frac{3 N_{\mathrm{P}}}{2048}+\frac{N_{\mathrm{L}} N_{\mathrm{P}}}{128}+\frac{N_{\mathrm{P}}^{2}}{512}\right) \gamma^{5} \\
& +\cdots
\end{aligned}
$$

Next is the sixth order of $\mathbf{0} \stackrel{0}{\rightarrow} \mathbf{0}$, for which, as mentioned in Sec. IV, the six-plaquette cube configurations as in Fig. 11 are to be considered. Let us first consider the contribution in the 2D case. Here three $+M \mathrm{~s}$ and $-M \mathrm{~s}$ cancel each other, and, depending on whether $M$ s sitting in one plaquette or not, we have 


$$
\begin{aligned}
& \left.\begin{array}{l}
0 \rightarrow 0,-M,-M,-M,+M,+M,+M \\
0,-M,-M,-M,+M,+M,+M \rightarrow 0
\end{array}\right\}: \\
& \quad C_{6}^{3}\left(6 N_{\mathrm{P}}\left(N_{\mathrm{P}}-1\right)\left(N_{\mathrm{P}}-2\right)+9 N_{\mathrm{P}}\left(N_{\mathrm{P}}-1\right)+N_{\mathrm{P}}\right) .
\end{aligned}
$$

The other is with 12 combinations of two $M$ s on one side and four $M \mathrm{~s}$ on another side; they cancel each other out:

$$
\left.\begin{array}{c}
0,-M,+M \rightarrow 0,-M,-M,+M,+M \\
0,-M,-M,+M,+M \rightarrow 0,-M,+M
\end{array}\right\}:
$$

The third term may contribute by cancellation between the four $M \mathrm{~s}$ and two $\delta \mathrm{s}$ in two sides:

$$
\left.\begin{array}{l}
0,-M,-M,+M,+M,+\delta,-\delta \rightarrow 0,+\delta,-\delta \\
0,+\delta,-\delta \rightarrow 0,-M,-M,+M,+M,+\delta,-\delta
\end{array}\right\}:
$$

The fourth may contribute by canceling two $M \mathrm{~s}$ in each side, and also $\delta \mathrm{s}$ in two sides as

$$
\begin{aligned}
& 0,+M,-M,+\delta,-\delta \rightarrow 0,+M,-M,+\delta,-\delta: \\
& \quad C_{2}^{1} C_{2}^{1} C_{2}^{1} N_{\mathrm{P}} N_{\mathrm{P}} N_{\mathrm{L}} .
\end{aligned}
$$

By this form, having possibilities for $+/-$ signs on the first side, we have the combinatorial factor

$$
\left.\begin{array}{l}
0,-M,+M,+\delta,+\delta,-\delta,-\delta \rightarrow 0,+\delta,+\delta,-\delta,-\delta \\
0,+\delta,+\delta,-\delta,-\delta \rightarrow 0,-M,+M,+\delta,+\delta,-\delta,-\delta,
\end{array}\right\}:
$$

The other is six $\delta$ s canceling each other out as follows:

$0,+\delta,+\delta,+\delta,-\delta-\delta,-\delta \rightarrow 0,+\delta,+\delta,+\delta,-\delta-\delta,-\delta:$

$$
C_{6}^{3}\left(6 N_{\mathrm{L}}\left(N_{\mathrm{L}}-1\right)\left(N_{\mathrm{L}}-2\right)+9 N_{\mathrm{L}}\left(N_{\mathrm{L}}-1\right)+N_{\mathrm{L}}\right) .
$$

In the last one, cancellation is between four link currents with the virtual loop current:

$$
\begin{aligned}
& \left.\begin{array}{l}
0, M,+\delta,+\delta,-\delta,-\delta \rightarrow 0, M+\delta,+\delta,-\delta,-\delta \\
0,-M,+\delta,+\delta,-\delta,-\delta \rightarrow 0,-M+\delta,+\delta,-\delta,-\delta
\end{array}\right\}: \\
& \quad C_{2}^{1} C_{2}^{1} C_{4}^{2} N_{\mathrm{P}}
\end{aligned}
$$

The graphical representation with all the above at this order will be as follows:

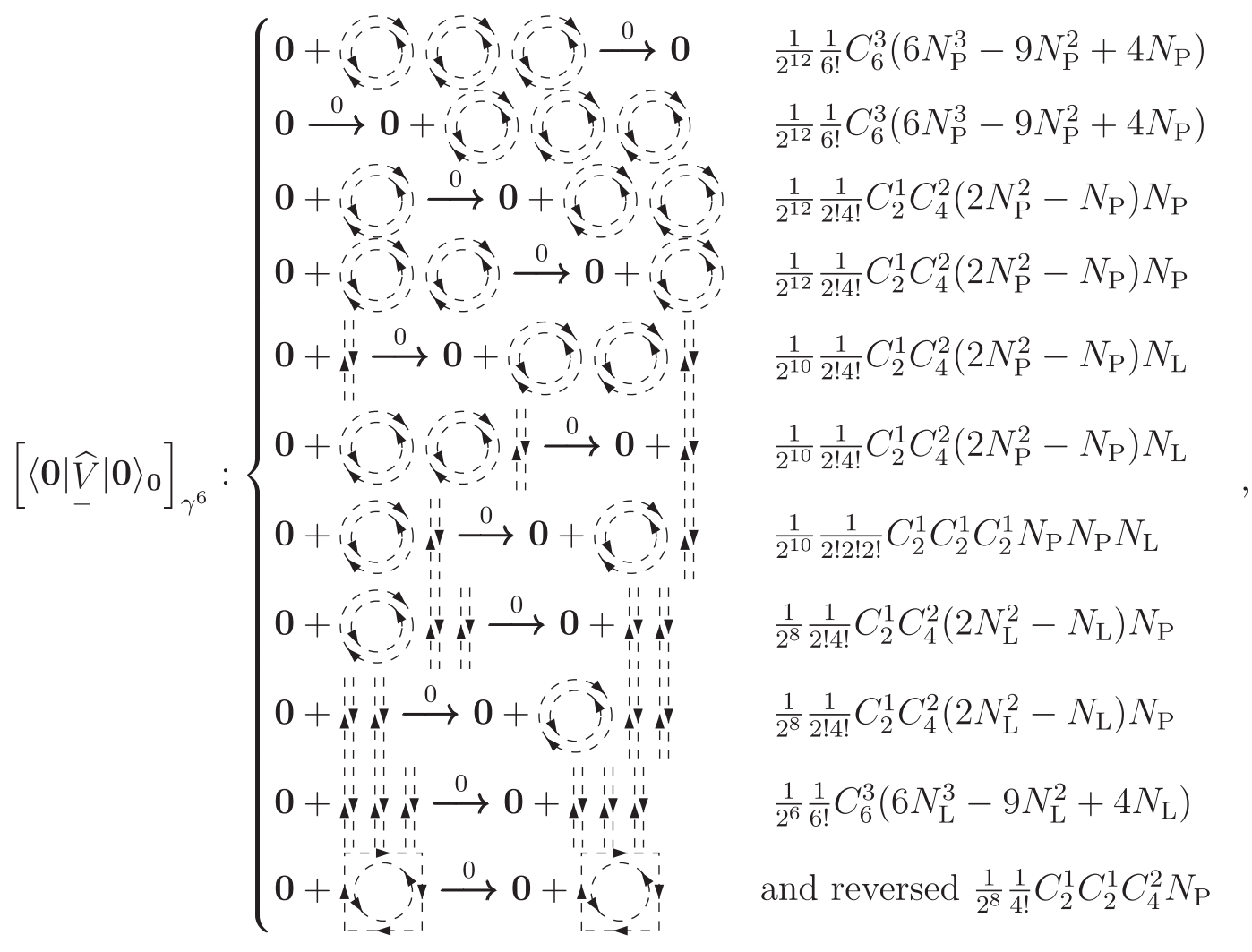


all together leading to

$$
\begin{aligned}
\langle\mathbf{0}|\hat{\underline{V}}| \mathbf{0}\rangle_{\mathbf{0}}= & 1+\left(\frac{N_{\mathrm{P}}}{8}+\frac{N_{\mathrm{L}}}{4}\right) \gamma^{2} \\
& +\left(-\frac{N_{\mathrm{L}}}{64}+\frac{N_{\mathrm{L}}^{2}}{32}-\frac{N_{\mathrm{P}}}{512}+\frac{N_{\mathrm{P}} N_{\mathrm{L}}}{32}+\frac{N_{\mathrm{P}}^{2}}{128}\right) \gamma^{4} \\
& +\left(\frac{N_{\mathrm{L}}}{576}-\frac{N_{\mathrm{L}}^{2}}{256}+\frac{N_{\mathrm{L}}^{3}}{384}+\frac{145 N_{\mathrm{P}}}{18432}-\frac{5 N_{\mathrm{L}} N_{\mathrm{P}}}{2048}+\frac{N_{\mathrm{L}}^{2} N_{\mathrm{P}}}{256}\right. \\
& \left.-\frac{N_{\mathrm{P}}^{2}}{4096}+\frac{N_{\mathrm{P}}^{2} N_{\mathrm{L}}}{512}+\frac{N_{\mathrm{P}}^{3}}{3072}\right) \gamma^{6}+\cdots
\end{aligned}
$$

As mentioned earlier, for the 3D lattice, the above result should be added by the contribution of cube configuration like that in Fig. 11. The numerical factor is known by cases (79) or (80), via (69). The last step is to find the relevant combinatorial factor that counts the possible ways that the cubic setup may take place. A simple inspection shows that in either the configuration in Fig. 11 or its all currents reversed version, there are three $+M \mathrm{~s}$ and three $-M \mathrm{~s}$, by the convention introduced for $\boldsymbol{M}$ matrix. This rises a $3 ! \times 3$ ! factor in which three $+M \mathrm{~s}$ and three $-M \mathrm{~s}$ can sit on cube facets. Also, the number of $\delta$ s containing six $M \mathrm{~s}$ by mentioned signs is $C_{6}^{3}$. As for the position of the cube, for the 3D periodic lattice with $N_{s}$ sites in each direction, the cube may sit in $N_{s}^{3}$ places. These all together determine the contribution of the cube configuration at sixth order by Eq. (69) as

$$
\begin{aligned}
{\left[\langle\mathbf{0}|\underline{\underline{V}}| \mathbf{0}\rangle_{\mathbf{0}}\right]_{\mathbb{Q}}^{3 \mathrm{~d}} } & =2 \times 2 \times 3 ! \times 3 ! C_{6}^{3} N_{s}^{3} \frac{1}{2^{12}} \frac{1}{6 !} \gamma^{6} \\
& =\frac{1}{2^{10}} N_{s}^{3} \gamma^{6} .
\end{aligned}
$$

In the previous section, examples of the nonvacuum block at the lowest orders were given by explicit $\delta$ expressions by Fourier integrals that matched with given rules. It is useful to see how the given rules work for these blocks at higher orders. At $\gamma^{3}$ order in $\boldsymbol{k}_{\mathbf{1}}$ block, we consider terms by the virtual current in this block for $\boldsymbol{q}^{\prime}=\boldsymbol{q}=\mathbf{0}$. For the first term, the possible combination would be the cancellation of $\boldsymbol{k}_{\mathbf{1}}$ and “ $-\delta$ " despite the presence of the virtual loop current

$$
\left.\begin{array}{l}
k_{1}-\delta \rightarrow k_{1}-\delta-M+M \\
k_{1}-\delta-M+M \rightarrow k_{1}-\delta
\end{array}\right\}: C_{2}^{1} N_{\mathrm{P}}
$$

For the second term, the possible ways would be three combinations of two $-\delta$ s and one $+\delta$, in which $\boldsymbol{k}_{\mathbf{1}}$ cancels one of $-\delta \mathrm{s}$, and two others cancel each other out. Depending on whether all $\delta$ s are in the same link or not, we have the combinatorial factor

$k_{1}-\delta-\delta+\delta \rightarrow k_{1}-\delta-\delta+\delta: C_{3}^{1}\left(2\left(N_{\mathrm{L}}-1\right)+1\right)$.

These can be represented as

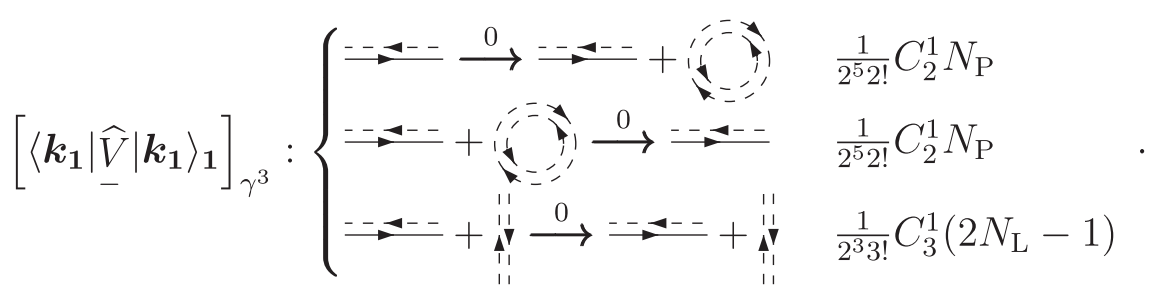

All together leading to

$$
\left\langle\boldsymbol{k}_{\mathbf{1}}|\underline{\hat{V}}| \boldsymbol{k}_{\mathbf{1}}\right\rangle_{\mathbf{1}}=\frac{\gamma}{2}+\left(-\frac{1}{16}+\frac{N_{\mathrm{L}}}{8}+\frac{N_{\mathrm{P}}}{16}\right) \gamma^{3}+\cdots .
$$

At $\gamma^{4}$ order with $\boldsymbol{q}^{\prime}=\mathbf{0}$ and $\boldsymbol{q}=\boldsymbol{q}_{\mathbf{1}}$ the graphical representations and combinatorial factors will be as follows:

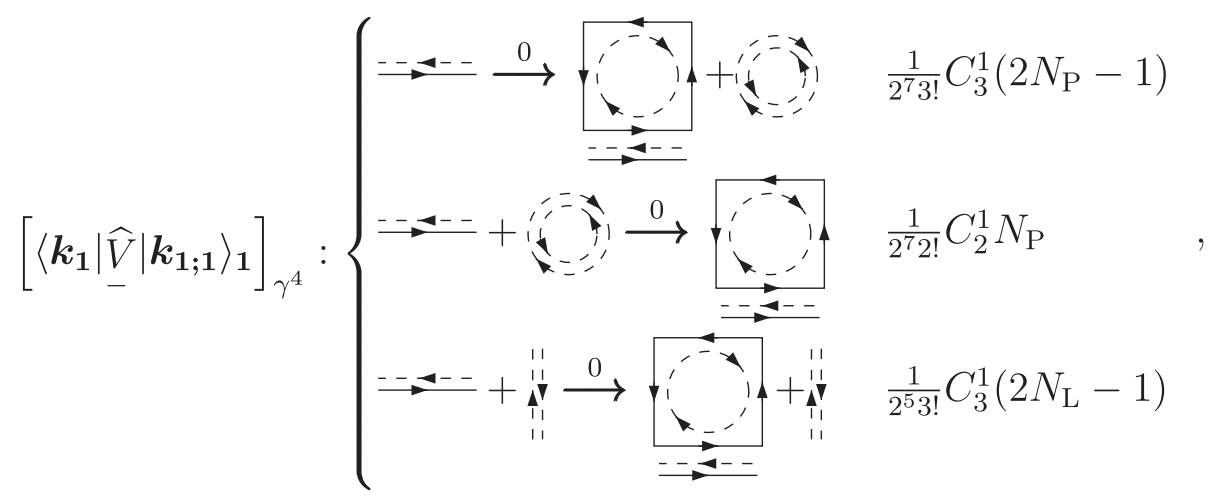


by which and (64) it ends with

$$
\left\langle\boldsymbol{k}_{\mathbf{1}}|\underline{\hat{V}}| \boldsymbol{k}_{\mathbf{1} ; \mathbf{1}}\right\rangle_{\mathbf{1}}=\frac{\gamma^{2}}{8}+\left(-\frac{5}{256}+\frac{N_{\mathrm{L}}}{32}+\frac{N_{\mathrm{P}}}{64}\right) \gamma^{4}+\cdots .
$$

It is easy to check that at order $\gamma^{5}$ the element by $\boldsymbol{q}^{\prime}=\boldsymbol{q}=\boldsymbol{q}_{\mathbf{1}}$ gets contributions as

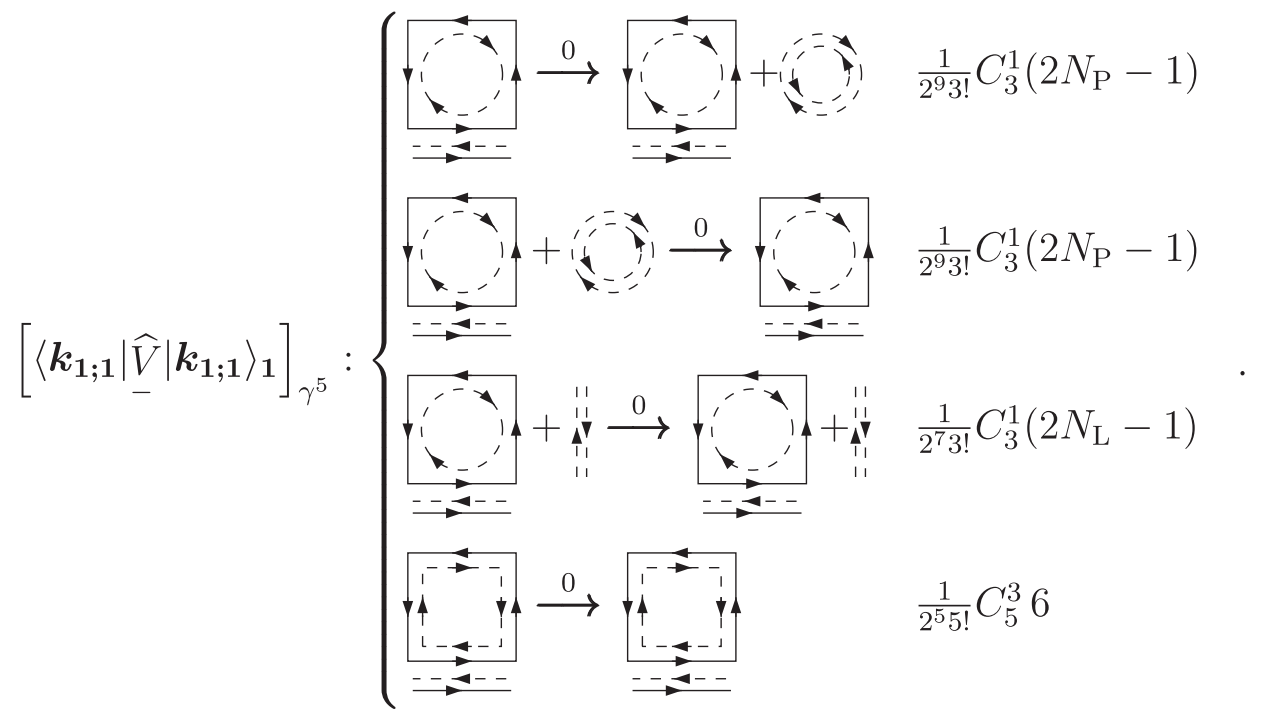

Using Eqs. (67) and (127) results in

$\left\langle\boldsymbol{k}_{\mathbf{1} ; \mathbf{1}}|\underline{\hat{V}}| \boldsymbol{k}_{\mathbf{1} ; \mathbf{1}}\right\rangle_{\mathbf{1}}=\frac{\gamma^{3}}{32}+\left(\frac{5}{512}+\frac{N_{\mathrm{L}}}{128}+\frac{N_{\mathrm{P}}}{256}\right) \gamma^{5}+\cdots$

\section{SPECTRUM IN A STRONG COUPLING LIMIT}

In the present section, it is shown how the expansion of the elements of the transfer-matrix in the Fourier basis can be used directly to calculate the energy spectrum in the strong coupling regime. In particular, here we calculate the ground state and the first excited energies using the expansion obtained for the transfer matrix of the U(1) model. The calculation is analytical, using simple matrix and quantum perturbation methods, and the eigenvalues of the mentioned states are calculated up to the fourth order in $\gamma$. To calculate far beyond this order, the numerical methods are needed to work with large matrices.

As it was seen in the previous sections, in the extreme strong coupling limit $\gamma=0$ all the matrix elements are zero, except $\langle\mathbf{0}|\underline{\hat{V}}| \mathbf{0}\rangle$, which is 1 . The $\gamma=0$ limit is considered as the unperturbed case, corresponding to the following eigenvalues for $\hat{V}^{0}$ :

$$
\gamma=0: v_{0}^{(0)}=1, \quad v_{q \neq 0}^{(0)}=0
$$

Using (17), the unperturbed energy values are obtained as $\varepsilon_{0}^{(0)}=0$ for the ground state and $\varepsilon_{q \neq 0}^{(0)} \rightarrow+\infty$ for all other states. The full transfer matrix, corresponding to a nonzero but small $\gamma$ is

$$
\underline{\hat{V}}=\hat{V}^{0}+\bar{V}
$$

It is useful to have the explicit expressions of perturbative corrections to the eigenvalues up to the fourth order in the present case

$$
\begin{gathered}
v_{q}^{(1)}=\bar{V}_{q q}, \\
v_{q}^{(2)}=\frac{\bar{V}_{q q^{\prime}}^{2}}{v_{q q^{\prime}}}, \\
v_{q}^{(3)}=\frac{\bar{V}_{q q^{\prime}} \bar{V}_{q^{\prime} q^{\prime \prime}} \bar{V}_{q^{\prime \prime} q}}{v_{q q^{\prime}} v_{q q^{\prime \prime}}}-\bar{V}_{q q} \frac{\bar{V}_{q q^{\prime}}^{2}}{v_{q q^{\prime}}^{2}}, \\
v_{q}^{(4)}=\frac{\bar{V}_{q q^{\prime}} \bar{V}_{q^{\prime} q^{\prime \prime}} \bar{V}_{q^{\prime \prime} q^{\prime \prime \prime}} \bar{V}_{q^{\prime \prime \prime} q}}{v_{q q^{\prime}} v_{q q^{\prime \prime}} v_{q q^{\prime \prime \prime}}}-\frac{\bar{V}_{q q^{\prime}}^{2}}{v_{q q^{\prime}}^{2}} \frac{\bar{V}_{q q^{\prime \prime}}^{2}}{v_{q q^{\prime \prime}}} \\
-2 \bar{V}_{q q} \frac{\bar{V}_{q q^{\prime}} \bar{V}_{q^{\prime} q^{\prime \prime}} \bar{V}_{q^{\prime \prime} q}}{v_{q q^{\prime}}^{2} v_{q q^{\prime \prime}}}+\bar{V}_{q q}^{2} \frac{\bar{V}_{q q^{\prime}}^{2}}{v_{q q^{\prime}}^{3}},
\end{gathered}
$$

in which $v_{q q^{\prime}}=v_{q}^{(0)}-v_{q^{\prime}}^{(0)}$ and $\bar{V}_{q q^{\prime}}=\left\langle\boldsymbol{q}|\bar{V}| \boldsymbol{q}^{\prime}\right\rangle$. The summations in the above expressions are understood over those values of $q^{\prime}, q^{\prime \prime}$, and $q^{\prime \prime \prime}$, for which the denominators do not vanish. It was mentioned at the end of Sec. II that the ground state of the U(1) model belongs to the vacuum 
block [14]. The lowest order of $\gamma$ in a matrix element was already obtained in Eq. (75). Up to the order $\gamma^{2}$, the only nonvanishing elements in the vacuum block are those with at most two units of loop currents in one or two plaquettes, namely

$$
\bar{V}_{0, \pm 1}=\frac{\gamma}{4}+\mathrm{O}\left(\gamma^{3}\right)
$$

$\bar{V}_{ \pm 1, \pm 1^{\prime} \text { or } \pm 1}, \bar{V}_{ \pm 1^{\prime}, \text { or } \mp 1}, \bar{V}_{0, \pm 2}, \bar{V}_{0, \pm 1 \pm 1}, \bar{V}_{0, \pm 1 \mp 1} \propto \gamma^{2}$.

We also have

$$
\bar{V}_{00}=\left(\frac{N_{\mathrm{P}}}{8}+\frac{N_{\mathrm{L}}}{4}\right) \gamma^{2}+\mathrm{O}\left(\gamma^{4}\right)
$$

Using Eq. (132), it is easily understood that at the order of $\gamma^{2}$ there are $2 N_{\mathrm{P}}$ elements $\bar{V}_{0, q}$ with $|q|=1$ that contribute to the ground state. Also by (129), in the perturbative corrections to the ground state we have $v_{0 q}=1-0=1$ in the denominator. All together, it is seen that up to the order $\gamma^{2}$

$$
\begin{aligned}
v_{0} & =1+\bar{V}_{00}+2 N_{\mathrm{P}} \bar{V}_{0, \pm 1}^{2}, \\
& =1+\frac{1}{4}\left(N_{\mathrm{P}}+N_{\mathrm{L}}\right) \gamma^{2}+\mathrm{O}\left(\gamma^{4}\right) .
\end{aligned}
$$

Further, using the well-known expression for the correction to the eigenvectors, we find for the ground state

$$
\begin{aligned}
\vec{v}_{0} & =(1,0,0, \ldots 0)+\sum_{|q|=1} \bar{V}_{0, q} \vec{q}, \\
& =(1, \underbrace{\frac{\gamma}{4}, \frac{\gamma}{4}, \ldots, \frac{\gamma}{4}}_{2 N_{\mathrm{P}}})+\mathrm{O}\left(\gamma^{2}\right) .
\end{aligned}
$$

Interestingly, we see that the expansion of the transfermatrix elements in the Fourier basis can be used to develop an expansion for the energy and eigenvector of the ground state. The physical meaning of the above vector based on the current states is simple. In the extreme strong limit $\gamma=0$, the ground state is simply the vacuum. For nonzero but small $\gamma$, the ground state is represented by a linear combination of the vacuum state and $2 N_{\mathrm{P}}$ states constructed by \pm 1 unit of loop current in one plaquette, such as Eq. (23) in Fig. 4.

It is instructive to find also the correction of order $\gamma^{2}$ to the ground state by a truncated version of the vacuum block as following

$$
\hat{\mathrm{V}}_{\mathrm{vac} \mid \gamma^{2}}=\left(\begin{array}{c|c}
\mathrm{A} & \mathrm{B} \\
\hline \mathrm{B}^{T} & \mathrm{C}
\end{array}\right),
$$

TABLE I. The sub-blocks in the truncated vacuum block.

\begin{tabular}{lcc}
\hline \hline Sub-block & Dimension & Elements \\
\hline A & $1 \times 1$ & $a=1+\bar{V}_{00}$ \\
$\mathrm{~B}$ & $1 \times 2 N_{\mathrm{P}}$ & $b=\bar{V}_{0, \pm 1}=\gamma / 4$ \\
$\mathrm{C}$ & $2 N_{\mathrm{P}} \times 2 N_{\mathrm{P}}$ & $c=\left\{\begin{array}{l}\bar{V}_{ \pm 1, \pm 1^{\prime} \text { or } \pm 1}=\gamma^{2} / 16 \\
\bar{V}_{ \pm 1, \mp 1^{\prime} \text { or } \mp 1}=\gamma^{2} / 16\end{array}\right.$ \\
\hline \hline
\end{tabular}

with $\mathrm{B}^{T}$ being the transpose of $\mathrm{B}$. The sub-blocks $\mathrm{A}, \mathrm{B}$, and $\mathrm{C}$ are introduced in Table I.

Fortunately, the eigenvalues and eigenvectors of Eq. (142) can be obtained analytically. For the eigenvalues we find

$$
\begin{gathered}
v_{0}=\frac{1}{2}\left(a+2 N_{\mathrm{P}} c+\sqrt{\left(a-2 N_{\mathrm{P}} c\right)^{2}+8 N_{\mathrm{P}} b^{2}}\right) \\
v_{1}=\frac{1}{2}\left(a+2 N_{\mathrm{P}} c-\sqrt{\left(a-2 N_{\mathrm{P}} c\right)^{2}+8 N_{\mathrm{P}} b^{2}}\right), \\
v_{i=2, \ldots, 2 N_{\mathrm{P}}+1}=0
\end{gathered}
$$

and the following for the ground-state eigenvector

$$
\vec{v}_{0}=(\frac{1}{2}\left(a-2 N_{\mathrm{P}} c+\sqrt{\left(a-2 N_{\mathrm{P}} c\right)^{2}+8 N_{\mathrm{P}} b^{2}}\right), \underbrace{b, b, \ldots, b}_{2 N_{\mathrm{P}}}) .
$$

Expanding Eqs. (143) and (146) up to $\gamma^{2}$, the expressions (139) and (141) are obtained. Also as a by-product, using Eq. (144), one also finds for the next eigenvalue in the vacuum block

$$
v_{1}=0 \times \gamma^{2}+\mathrm{O}\left(\gamma^{4}\right)
$$

This is of the order $\gamma^{4}$, so the first excited energy is not in the vacuum block. As we will see, there are blocks with eigenvalues of order $\gamma$, which are in fact the first excited energies of the model. Before considering the excited states, let us consider the ground-state energy at the order $\gamma^{4}$. First, we have the element

$$
\begin{aligned}
\bar{V}_{00}= & \left(\frac{N_{\mathrm{P}}}{8}+\frac{N_{\mathrm{L}}}{4}\right) \gamma^{2} \\
& +\left(-\frac{N_{\mathrm{L}}}{64}+\frac{N_{\mathrm{L}}^{2}}{32}-\frac{N_{\mathrm{P}}}{512}+\frac{N_{\mathrm{P}} N_{\mathrm{L}}}{32}+\frac{N_{\mathrm{P}}^{2}}{128}\right) \gamma^{4} \\
& +\mathrm{O}\left(\gamma^{6}\right)
\end{aligned}
$$

and the elements 
$\bar{V}_{0, q}=\frac{\gamma}{4}+\left(\frac{-1}{128}+\frac{N_{\mathrm{P}}}{32}+\frac{N_{\mathrm{L}}}{16}\right) \gamma^{3}+\mathrm{O}\left(\gamma^{5}\right), \quad|q|=1$,

$\bar{V}_{0, q}=\frac{\gamma^{2}}{32}+\left(\frac{-1}{768}+\frac{N_{\mathrm{L}}}{128}+\frac{N_{\mathrm{P}}}{256}\right) \gamma^{4}+\mathrm{O}\left(\gamma^{6}\right), \quad|q|=2$,

each having $2 N_{\mathrm{P}}$ cases, by considering \pm signs. Second, there are the elements

$\bar{V}_{q, q}=\frac{\gamma^{2}}{16}+\left(\frac{15}{256}+\frac{N_{\mathrm{L}}}{64}+\frac{N_{\mathrm{P}}}{128}\right) \gamma^{4}+\mathrm{O}\left(\gamma^{6}\right), \quad|q|=1$,

$\bar{V}_{q,-q}=\frac{\gamma^{2}}{16}+\left(\frac{-1}{256}+\frac{N_{\mathrm{L}}}{64}+\frac{N_{\mathrm{P}}}{128}\right) \gamma^{4}+\mathrm{O}\left(\gamma^{6}\right), \quad|q|=1$,

each with $2 N_{\mathrm{P}}$ numbers. The other relevant elements are

$$
\begin{aligned}
\bar{V}_{q, q^{\prime}} & =\frac{\gamma^{2}}{16}+\left(-\frac{1}{256}+\frac{N_{\mathrm{L}}}{64}+\frac{N_{\mathrm{P}}}{128}\right) \gamma^{4}+\mathrm{O}\left(\gamma^{6}\right), \\
q & \neq q^{\prime}, \quad|q|=\left|q^{\prime}\right|=1, \\
\bar{V}_{0, q q^{\prime}} & =\frac{\gamma^{2}}{16}+\left(-\frac{1}{256}+\frac{N_{\mathrm{L}}}{64}+\frac{N_{\mathrm{P}}}{128}\right) \gamma^{4}+\mathrm{O}\left(\gamma^{6}\right), \\
q & \neq q^{\prime}, \quad|q|=\left|q^{\prime}\right|=1,
\end{aligned}
$$

with $4 N_{\mathrm{P}}\left(N_{\mathrm{P}}-1\right)$ and $2 N_{\mathrm{P}}\left(N_{\mathrm{P}}-1\right)$ numbers, respectively. There are other elements at the order $\gamma^{3}$ such as

$\bar{V}_{0, \pm 3}, \quad \bar{V}_{0, \pm 1 \pm 2}, \quad \bar{V}_{ \pm 1, \pm 2}, \quad \bar{V}_{0, \pm 1 \pm 1 \pm 1}, \quad \bar{V}_{ \pm 1, \pm 1 \pm 1}$

and also at the order $\gamma^{4}$ :

$\bar{V}_{0, \pm 4}, \bar{V}_{ \pm 1, \pm 3}, \bar{V}_{0, \pm 1 \pm 3}, \bar{V}_{ \pm 2, \pm 2}, \bar{V}_{0, \pm 2 \pm 2}, \bar{V}_{1 \pm, \pm 1 \pm 2}$,

$\bar{V}_{0, \pm 1 \pm 1 \pm 2} ; \bar{V}_{0, \pm 1 \pm 1 \pm 1 \pm 1}, \bar{V}_{ \pm 1, \pm 1 \pm 1 \pm 1}, \bar{V}_{ \pm 1 \pm 1, \pm 1 \pm 1}$.

However, a simple inspection based on corrections (131)(134) shows that none of the terms of the order $\gamma^{3}$ in Eq. (155), and none of $\gamma^{4} \mathrm{~s}$ in Eqs. (150)-(156) contribute at the order $\gamma^{4}$ to the ground state. It is also easy to check that the correction at the order $\gamma^{3}$ vanishes. So the next correction is of the order $\gamma^{4}$, which finds contributions as following

$$
\begin{aligned}
v_{0}^{(1)} & =\bar{V}_{00}-\left(\gamma^{2} \text { order }\right) \\
& =\left(-\frac{N_{\mathrm{L}}}{64}+\frac{N_{\mathrm{L}}^{2}}{32}-\frac{N_{\mathrm{P}}}{512}+\frac{N_{\mathrm{P}} N_{\mathrm{L}}}{32}+\frac{N_{\mathrm{P}}^{2}}{128}\right) \gamma^{4},
\end{aligned}
$$

$$
\begin{aligned}
v_{0}^{(2)}= & 2 N_{\mathrm{P}} \bar{V}_{0,1}^{2}+2 N_{\mathrm{P}} \bar{V}_{0,2}^{2}+2 N_{\mathrm{P}}\left(N_{\mathrm{P}}-1\right) \bar{V}_{0,11}^{2}, \\
= & 2 N_{\mathrm{P}} \times 2 \frac{\gamma}{4}\left(\frac{-1}{128}+\frac{N_{\mathrm{P}}}{32}+\frac{N_{\mathrm{L}}}{16}\right) \gamma^{3}+2 N_{\mathrm{P}}\left(\frac{\gamma^{2}}{32}\right)^{2} \\
& +2 N_{\mathrm{P}}\left(N_{\mathrm{P}}-1\right)\left(\frac{\gamma^{2}}{16}\right)^{2}, \\
= & \left(\frac{-7 N_{\mathrm{P}}}{512}+\frac{N_{\mathrm{L}} N_{\mathrm{P}}}{16}+\frac{5 N_{\mathrm{P}}^{2}}{128}\right) \gamma^{4}, \\
v_{0}^{(3)}= & 2 \times 2 N_{\mathrm{P}} \bar{V}_{0,1} \bar{V}_{1,1} \bar{V}_{0,1}+4 N_{\mathrm{P}}\left(N_{\mathrm{P}}-1\right) \bar{V}_{0,1} \bar{V}_{1,1^{\prime}} \bar{V}_{0,1^{\prime}} \\
& -2 N_{\mathrm{P}} \bar{V}_{00} \bar{V}_{0,1}^{2}, \\
= & 4 N_{\mathrm{P}}\left(\frac{\gamma}{4}\right)^{2}\left(\frac{\gamma^{2}}{16}\right)+4 N_{\mathrm{P}}\left(N_{\mathrm{P}}-1\right)\left(\frac{\gamma}{4}\right)^{2}\left(\frac{\gamma^{2}}{16}\right) \\
& -2 N_{\mathrm{P}}\left(\frac{\gamma}{4}\right)^{2}\left(\frac{N_{\mathrm{L}}}{4}+\frac{N_{\mathrm{P}}}{8}\right) \gamma^{2}, \\
= & -\frac{N_{\mathrm{L}} N_{\mathrm{P}}}{32} \gamma^{4},
\end{aligned}
$$

$v_{0}^{(4)}=-2 N_{\mathrm{P}} \times 2 N_{\mathrm{P}} \bar{V}_{0,1}^{2} \bar{V}_{0,1}^{2}=-4 N_{\mathrm{P}}^{2}\left(\frac{\gamma}{4}\right)^{2}\left(\frac{\gamma}{4}\right)^{2}=-\frac{N_{\mathrm{P}}^{2}}{64} \gamma^{4}$.

All together we have for the ground-state energy

$$
\begin{aligned}
v_{0}= & 1+\frac{1}{4}\left(N_{\mathrm{P}}+N_{\mathrm{L}}\right) \gamma^{2} \\
& +\left(\frac{-N_{\mathrm{L}}}{64}+\frac{N_{\mathrm{L}}^{2}}{32}-\frac{N_{\mathrm{P}}}{64}+\frac{N_{\mathrm{L}} N_{\mathrm{P}}}{16}+\frac{N_{\mathrm{P}}^{2}}{32}\right) \gamma^{4} \\
& +\mathrm{O}\left(\gamma^{6}\right) .
\end{aligned}
$$

Now let us consider the first excited state by the transfer matrix of the model. Using the previously obtained expansions, the state with one unit of current on one link, such as $\boldsymbol{k}_{\mathbf{1}}$ of Eq. (24), leads to a diagonal element linear in $\gamma$ at the lowest order as Eq. (124). The first element of the block up to order $\gamma^{5}$, expressed in the notation of present section, is

$$
\begin{aligned}
\bar{V}_{00}^{k_{1}}= & \frac{\gamma}{2}+\left(-\frac{1}{16}+\frac{N_{\mathrm{L}}}{8}+\frac{N_{\mathrm{P}}}{16}\right) \gamma^{3} \\
& +\left(\frac{7}{384}-\frac{3 N_{\mathrm{L}}}{128}+\frac{N_{\mathrm{L}}^{2}}{64}-\frac{9 N_{\mathrm{P}}}{1024}+\frac{N_{\mathrm{L}} N_{\mathrm{P}}}{64}+\frac{N_{\mathrm{P}}^{2}}{256}\right) \gamma^{5} \\
& +\mathrm{O}\left(\gamma^{7}\right) .
\end{aligned}
$$

So the first excited states belongs to the blocks with current states such as $\boldsymbol{k}_{\mathbf{1}}$. Considering \pm 1 units of current on one link as the representative state, the number of such blocks are $2 N_{\mathrm{L}}$. So the first excited energy has a degeneracy of $2 N_{\mathrm{L}}$. In fact, finding the corrections to the first excited energy is simple and can be done in the same way done for 
the ground state. Factoring out $\gamma / 2$ from the elements of the $\boldsymbol{k}_{\mathbf{1}}$ block, the very same calculation for the ground state can be repeated. Similar to the method used for the vacuum block, we have the following elements in the $\boldsymbol{k}_{\mathbf{1}}$ block

$$
\begin{gathered}
\bar{V}_{00}^{k_{1}}=\frac{\gamma}{2}\left(1+\left(-\frac{1}{8}+\frac{N_{\mathrm{L}}}{4}+\frac{N_{\mathrm{P}}}{8}\right) \gamma^{2}+\mathrm{O}\left(\gamma^{4}\right)\right), \\
\bar{V}_{0, \pm 1}^{k_{1}}=\frac{\gamma}{2}\left(\frac{\gamma}{4}+\mathrm{O}\left(\gamma^{3}\right)\right),
\end{gathered}
$$

in which we find for the first excited energy

$$
\begin{aligned}
v_{0}^{k_{1}} & =\frac{\gamma}{2}\left[1+\left(-\frac{1}{8}+\frac{N_{\mathrm{L}}}{4}+\frac{N_{\mathrm{P}}}{8}\right) \gamma^{2}+2 N_{\mathrm{P}}\left(\frac{\gamma}{4}\right)^{2}\right]+\mathrm{O}\left(\gamma^{5}\right), \\
& =\frac{\gamma}{2}+\left(-\frac{1}{16}+\frac{N_{\mathrm{L}}}{8}+\frac{N_{\mathrm{P}}}{8}\right) \gamma^{3}+\mathrm{O}\left(\gamma^{5}\right)
\end{aligned}
$$

The gap of energy by the model can be calculated by the ground-state energy (161) and the above as the excited one. The eigenvector of the energy (166) can be obtained using the perturbation method as well

$$
\begin{aligned}
\vec{v}_{0}^{k_{1}} & =(1,0,0, \ldots 0)+\frac{1}{\gamma / 2} \sum_{|q|=1} \bar{V}_{0, q}^{k_{1}} \vec{q}, \\
& =(1, \underbrace{\frac{\gamma}{4}, \frac{\gamma}{4}, \ldots, \frac{\gamma}{4}}_{2 N_{\mathrm{P}}})+\mathrm{O}\left(\gamma^{2}\right) .
\end{aligned}
$$

It is noticed that the vector is exactly in the form of Eq. (141) in the vacuum block, however, regarding the current states, with a different physical meaning. Here, the eigenvector is the linear combination of the $\boldsymbol{k}_{1}$ state and $2 N_{\mathrm{P}}$ states constructed by the $\boldsymbol{k}_{\mathbf{1}}$ state added by \pm 1 unit of loop current in one plaquette, such as Fig. 7.

The next correction can be obtained along the lines of Eqs. (157)-(160) but in the $\boldsymbol{k}_{\mathbf{1}}$ block, leading to

$$
\begin{aligned}
v_{0}^{k_{1}}= & \frac{\gamma}{2}+\left(\frac{-1}{16}+\frac{N_{\mathrm{L}}}{8}+\frac{N_{\mathrm{P}}}{8}\right) \gamma^{3} \\
& +\left(\frac{7}{384}-\frac{3 N_{\mathrm{L}}}{128}+\frac{N_{\mathrm{L}}^{2}}{64}-\frac{3 N_{\mathrm{P}}}{128}+\frac{N_{\mathrm{L}} N_{\mathrm{P}}}{32}+\frac{N_{\mathrm{P}}^{2}}{64}\right) \gamma^{5} \\
& +\mathrm{O}\left(\gamma^{7}\right) .
\end{aligned}
$$

The above procedure to find the eigenvalues can be used for the other blocks as well. For later use in the next section, we present the lowest eigenvalue in the $\boldsymbol{k}_{\mathbf{2}}$ block,

$$
\begin{aligned}
v_{0}^{k_{2}}= & \frac{\gamma^{2}}{8}+\left(-\frac{1}{48}+\frac{N_{\mathrm{L}}}{32}+\frac{N_{\mathrm{P}}}{32}\right) \gamma^{4} \\
& +\left(\frac{23}{3072}-\frac{11 N_{\mathrm{L}}}{1536}+\frac{N_{\mathrm{L}}^{2}}{256}-\frac{11 N_{\mathrm{P}}}{1536}+\frac{N_{\mathrm{L}} N_{\mathrm{P}}}{128}+\frac{N_{\mathrm{P}}^{2}}{256}\right) \gamma^{6} \\
& +\mathrm{O}\left(\gamma^{8}\right),
\end{aligned}
$$

the $\boldsymbol{k}_{11^{\prime}}$ block,

$$
\begin{aligned}
v_{0}^{k_{11^{\prime}}}= & \frac{\gamma^{2}}{4}+\left(-\frac{1}{16}+\frac{N_{\mathrm{L}}}{16}+\frac{N_{\mathrm{P}}}{16}\right) \gamma^{4} \\
& +\left(\frac{7}{384}-\frac{5 N_{\mathrm{L}}}{256}+\frac{N_{\mathrm{L}}^{2}}{128}-\frac{5 N_{\mathrm{P}}}{256}+\frac{N_{\mathrm{L}} N_{\mathrm{P}}}{64}+\frac{N_{\mathrm{P}}^{2}}{128}\right) \gamma^{6} \\
& +\mathrm{O}\left(\gamma^{8}\right)
\end{aligned}
$$

and the $\boldsymbol{k}_{\mathbf{3}}$ block,

$$
\begin{aligned}
v_{0}^{k_{3}}= & \frac{\gamma^{3}}{48}+\left(-\frac{1}{256}+\frac{N_{\mathrm{L}}}{192}+\frac{N_{\mathrm{P}}}{192}\right) \gamma^{5} \\
& +\left(\frac{17}{10240}-\frac{N_{\mathrm{L}}}{768}+\frac{N_{\mathrm{L}}^{2}}{1536}-\frac{N_{\mathrm{P}}}{768}+\frac{N_{\mathrm{L}} N_{\mathrm{P}}}{768}+\frac{N_{\mathrm{P}}^{2}}{1536}\right) \gamma^{7} \\
& +\mathrm{O}\left(\gamma^{9}\right) .
\end{aligned}
$$

\section{LATTICE SIZE AND OBSERVABLE VALUES}

In the presented expansions of the transfer-matrix elements and eigenvalues, all of the terms except the first ones have positive powers of $N_{\mathrm{P}}$ and $N_{\mathrm{L}}$. So for an infinite lattice, in which $N_{\mathrm{P}}$ and $N_{\mathrm{L}}$ go to infinity, a proper interpretation of the expansions is necessary. The purpose of this section is twofold: first, to show how the presence of the mentioned numbers in the expansions is expected, and, second, to present a proper interpretation of the resulted expansions, which are formally divergent in the large lattice limit.

Let us consider the matrix-element $\left\langle\boldsymbol{k}^{\prime}|\hat{V}| \boldsymbol{k}\right\rangle$ representing the transition $\boldsymbol{k}^{\prime} \rightarrow \boldsymbol{k}$. It is shown that in the strong coupling regime, the $\gamma$ expansion of this matrix element is represented by virtual occurrences of loop and link currents that transform both states to the vacuum. The weight of each virtual current in expansions is $\gamma$. The first term represents the lowest number of virtual currents, in definite links and plaquettes, necessary to perform the mentioned transform to the vacuum. So, no $N_{\mathrm{L}}$ and $N_{\mathrm{P}}$ is expected in the first term. The other terms, however, represent all virtual currents in any link and plaquettes that may contribute to the transform. As a result, the number of links and plaquettes enter the expansions. As seen in the previous examples, this is exactly the way that powers of $N_{\mathrm{P}}$ and $N_{\mathrm{L}}$ appear in the expansions.

The way that $N_{\mathrm{L}}$ and $N_{\mathrm{P}}$ appear in the expansions provides a physical basis to treat the behavior of expansions in the infinite lattice limit. In particular, due to the 
mentioned role by the virtual currents in the transitions, a distinguished role is expected to be given to the element $\langle\mathbf{0}|\hat{V}| \mathbf{0}\rangle$ as the amplitude of the VTV transition. The reason is that the virtual events that derive the VTV transition are expected to take place in the transitions between other current states as well, leading to the appearance of $N_{\mathrm{L}}$ and $N_{\mathrm{P}}$ in the expansions. As a specific example, let us consider a $2 \mathrm{D}$ lattice, in which $N_{\mathrm{L}}=2 N_{\mathrm{P}}$. By replacing $N_{\mathrm{L}}$ by $N_{\mathrm{P}}$, the VTV transition (119) in the 2D lattice finds the following form

$$
\langle\mathbf{0}|\underline{\hat{V}}| \mathbf{0}\rangle_{\mathbf{0}}^{2 \mathrm{~d}}=1+\left(\frac{5 \gamma^{2}}{8}-\frac{17 \gamma^{4}}{512}+\frac{209 \gamma^{6}}{18432}\right) N_{\mathrm{P}}+\left(\frac{25 \gamma^{4}}{128}-\frac{85 \gamma^{6}}{4096}\right) N_{\mathrm{P}}^{2}+\left(\frac{125 \gamma^{6}}{3072}\right) N_{\mathrm{P}}^{3}+\cdots
$$

Based on the above explanations, one expects that a footprint of the VTV transition can be traced in other transitions. In particular, it is seen that the ratio of the other matrix elements to the VTV one is independent of $N_{\mathrm{P}}$. As examples in the vacuum block, we have the following for the 2D lattice:

$$
\begin{gathered}
\langle\mathbf{0}|\underline{\hat{V}}| \mathbf{1}\rangle_{\mathbf{0}}=\langle\mathbf{0}|\underline{\hat{V}}| \mathbf{0}\rangle \cdot \frac{\gamma}{4}\left(1-\frac{\gamma^{2}}{32}+\frac{49 \gamma^{4}}{768}-\frac{7691 \gamma^{6}}{196608}+\cdots\right), \\
\langle\mathbf{1}|\hat{\underline{V}}| \mathbf{1}\rangle_{\mathbf{0}}=\langle\mathbf{0}|\underline{\underline{V}}| \mathbf{0}\rangle \cdot \frac{\gamma^{2}}{16}\left(1+\frac{15 \gamma^{2}}{16}-\frac{1525 \gamma^{4}}{3072}+\frac{22861 \gamma^{6}}{98304}+\cdots\right), \\
\langle\mathbf{0}|\hat{\underline{V}}| \mathbf{2}\rangle_{\mathbf{0}}=\langle\mathbf{0}|\hat{\underline{V}}| \mathbf{0}\rangle \cdot \frac{\gamma^{2}}{32}\left(1-\frac{\gamma^{2}}{24}+\frac{1163 \gamma^{4}}{6144}-\frac{9073 \gamma^{6}}{81920}+\cdots\right), \\
\langle\mathbf{0}|\underline{\hat{V}}| \mathbf{3}\rangle_{\mathbf{0}}=\langle\mathbf{0}|\underline{\hat{V}}| \mathbf{0}\rangle \cdot \frac{\gamma^{3}}{384}\left(1-\frac{3 \gamma^{2}}{64}+\frac{1941 \gamma^{4}}{10240}+\frac{168611 \gamma^{6}}{1474560}+\cdots\right),
\end{gathered}
$$

in which we see that the $N_{\mathrm{P}}$ dependence is totally factored out as the VTV transition element. As more examples by composite current vectors in the vacuum block we have

$$
\begin{gathered}
\langle\mathbf{0}|\underline{\hat{V}}| \mathbf{1}, \mathbf{1}\rangle_{\mathbf{0}}=\langle\mathbf{0}|\hat{\underline{V}}| \mathbf{0}\rangle \cdot \frac{\gamma^{2}}{16}\left(1-\frac{\gamma^{2}}{16}+\frac{203 \gamma^{4}}{3072}+\frac{1325 \gamma^{6}}{98304}+\cdots\right), \\
\langle\mathbf{0}|\underline{\hat{V}}| \mathbf{1}, \mathbf{2}\rangle_{\mathbf{0}}=\langle\mathbf{0}|\hat{\underline{V}}| \mathbf{0}\rangle \cdot \frac{\gamma^{3}}{128}\left(1-\frac{7 \gamma^{2}}{96}+\frac{137 \gamma^{4}}{2048}+\frac{73319 \gamma^{6}}{1474560}+\cdots\right) .
\end{gathered}
$$

The above observation is expected to be true in blocks other than the vacuum block as well. As examples, from the $\boldsymbol{k}_{\mathbf{1}}$ and $\boldsymbol{k}_{\mathbf{2}}$ blocks we have

$$
\begin{aligned}
& \left\langle\boldsymbol{k}_{\mathbf{1}}|\underline{\hat{V}}| \boldsymbol{k}_{\mathbf{1}}\right\rangle_{\mathbf{1}}=\langle\mathbf{0}|\underline{\hat{V}}| \mathbf{0}\rangle \cdot \frac{\gamma}{2}\left(1-\frac{\gamma^{2}}{8}+\frac{7 \gamma^{4}}{192}-\frac{25 \gamma^{6}}{1536}+\cdots\right), \\
& \left\langle\boldsymbol{k}_{\mathbf{1}}|\underline{\hat{V}}| \boldsymbol{k}_{\mathbf{1} ; \mathbf{1}}\right\rangle_{\mathbf{1}}=\langle\mathbf{0}|\underline{\hat{V}}| \mathbf{0}\rangle \cdot \frac{\gamma^{2}}{8}\left(1-\frac{5 \gamma^{2}}{32}+\frac{25 \gamma^{4}}{384}-\frac{6619 \gamma^{6}}{196608}+\cdots\right), \\
& \left\langle\boldsymbol{k}_{\mathbf{1} ; \mathbf{1}}|\underline{\hat{V}}| \boldsymbol{k}_{\mathbf{1 ; 1}}\right\rangle_{\mathbf{1}}=\langle\mathbf{0}|\underline{\hat{V}}| \mathbf{0}\rangle \cdot \frac{\gamma^{3}}{32}\left(1+\frac{5 \gamma^{2}}{16}-\frac{721 \gamma^{4}}{3072}+\frac{8369 \gamma^{6}}{98304}+\cdots\right), \\
& \left\langle\boldsymbol{k}_{\mathbf{2}}|\underline{\hat{V}}| \boldsymbol{k}_{\mathbf{2}}\right\rangle_{\mathbf{2}}=\langle\mathbf{0}|\underline{\hat{V}}| \mathbf{0}\rangle \cdot \frac{\gamma^{2}}{8}\left(1-\frac{\gamma^{2}}{6}+\frac{11 \gamma^{4}}{384}-\frac{11 \gamma^{6}}{960}+\cdots\right), \\
& \left\langle\boldsymbol{k}_{\mathbf{2}}|\hat{\hat{V}}| \boldsymbol{k}_{\mathbf{2} ; \mathbf{1}}\right\rangle_{\mathbf{2}}=\langle\mathbf{0}|\hat{\underline{V}}| \mathbf{0}\rangle \cdot \frac{\gamma^{3}}{32}\left(1-\frac{19 \gamma^{2}}{96}+\frac{55 \gamma^{4}}{768}-\frac{103957 \gamma^{6}}{2949120}+\cdots\right), \\
& \left\langle\boldsymbol{k}_{\mathbf{2} ; \mathbf{1}}|\underline{\hat{V}}| \boldsymbol{k}_{\mathbf{2} ; \mathbf{1}}\right\rangle_{\mathbf{2}}=\langle\mathbf{0}|\underline{\hat{V}}| \mathbf{0}\rangle \cdot \frac{\gamma^{4}}{128}\left(1+\frac{5 \gamma^{2}}{48}-\frac{421 \gamma^{4}}{3072}+\frac{75107 \gamma^{6}}{1474560}+\cdots\right) \text {. }
\end{aligned}
$$


It is interesting that the above expressions are valid for any value of $N_{\mathrm{P}}$. Despite the fact that the whole dependence on $N_{\mathrm{P}}$ can be extracted equally from all elements, it does not make them finite in the large lattice limit. The basic idea to define physically finite values comes from the field theory approach to Feynman diagrams. As mentioned before, the present diagrammatic expansion has features similar to the those of the Feynman diagrams of perturbative quantum field theory. Here we see another example of these common features. The $n$-point functions in the field theory approach supposedly contain vacuum diagrams; those with no external legs originated from the theory with no source term. Having no external legs, the vacuum diagrams are the only ones that contribute to the VTV transition. The absence of external legs has two important consequences. First, the vacuum diagrams are infinite, as they can take place in the infinite extent of the space $[18,19]$. Second, the vacuum diagrams contribute equally as a multiplicative factor to all transitions [18-20]. Hence, the total contribution of these diagrams can be factored out from the $S$-matrix elements, leaving only physically observable contributions to the transitions [18-20]. In the field theory approach, the contribution by the vacuum diagrams is extracted from the $n$-point functions by defining the normalized generating functional, through dividing the path-integral expression with source " $J$ " by the sourceless one [20]

$$
Z[J]=\frac{\int \mathscr{D} \phi \exp \left[\mathrm{i} \int d x(\mathscr{L}+J \phi)\right]}{\int \mathscr{D} \phi \exp \left[\mathrm{i} \int d x \mathscr{L}\right]} .
$$

It then can be shown that the $n$-point functions derived from the normalized functional are free from the vacuum diagrams $[19,20]$. In a quite similar way, we see that the VTV contribution can be extracted from the present strong coupling expansion of matrix elements, leaving a finite physical transition amplitude between the current states. As mentioned in Sec. III, the current vector $\boldsymbol{k}$ plays the role of the source. The origin of the divergent behavior of $\langle\mathbf{0}|\underline{\underline{V}}| \mathbf{0}\rangle$ in the infinite lattice limit is the same as its counterpart in the field theory approach.

We see that, by extracting $\langle\mathbf{0}|\underline{\hat{V}}| \mathbf{0}\rangle$ from the transfer matrix, all elements become independent of $N_{\mathrm{P}}$. However, that is not enough to guarantee that the eigenvalues are independent of $N_{\mathrm{P}}$. This can be checked explicitly by the eigenvalues obtained in the previous section. The reason for this is simply that the number of off-diagonal elements contributing to an eigenvalue may depend on $N_{\mathrm{P}}$. We already have seen examples of this in the previous section, for example Eqs. (138) and (158)-(160). Theoretically, for a system with finite energy density we expect infinite energy in the infinite size limit [19]. So, there is no surprise about the divergent behavior of the obtained eigenvalues. However, it is still expected that the observable values related to energy would be finite. Fortunately the solution is quite known that, as far as measurements are concerned, the relevant quantity is the energy difference rather than the energy itself. In our case, using $v_{i}=\exp \left(-a \varepsilon_{i}\right)$, it is enough to check the behavior of the ratio $v_{i} / v_{0}=$ $\exp \left[-a\left(\varepsilon_{i}-\varepsilon_{0}\right)\right]$. If the mentioned ratio is independent of $N_{\mathrm{P}}$, then the difference between every two energies will be too. As explicit examples, for eigenvalues obtained in the $\boldsymbol{k}_{1}, \boldsymbol{k}_{\mathbf{2}}, \boldsymbol{k}_{11^{\prime}}$, and $\boldsymbol{k}_{\mathbf{3}}$ blocks,

$$
\begin{gathered}
\frac{v_{0}^{k_{1}}}{v_{0}}=\frac{\gamma}{2}\left(1-\frac{\gamma^{2}}{8}+\frac{7 \gamma^{4}}{192}+\cdots\right), \\
\frac{v_{0}^{k_{2}}}{v_{0}}=\frac{\gamma^{2}}{8}\left(1-\frac{\gamma^{2}}{6}+\frac{23 \gamma^{4}}{384}+\cdots\right), \\
\frac{v_{0}^{k_{11^{\prime}}}}{v_{0}}=\frac{\gamma^{2}}{4}\left(1-\frac{\gamma^{2}}{4}+\frac{7 \gamma^{4}}{96}+\cdots\right), \\
\frac{v_{0}^{k_{3}}}{v_{0}}=\frac{\gamma^{3}}{48}\left(1-\frac{3 \gamma^{2}}{16}+\frac{51 \gamma^{4}}{640}+\cdots\right),
\end{gathered}
$$

in which all ratios are independent of $N_{\mathrm{P}}$. By the above observation, to excite the system from the ground state a finite amount of energy, independent of the lattice size, is needed.

\section{CONCLUSION AND DISCUSSION}

The formulation of the transfer matrix of the U(1) lattice model in the field Fourier basis was studied. It was discussed in detail how the states in the Fourier basis correspond to quantized currents on links. The constraint to have a nonvanishing element by two states was shown to be in fact the condition that, as a lattice version of current conservation, the two states differ in loop currents circulating inside plaquettes. These features provide a basis to develop the strong coupling expansion and its diagrammatic representation for the elements of the transfer matrix in the field Fourier basis. Each term of the expansion represents the occurrence of virtual loop and link currents that transform the initial and final states to the vacuum state. Accordingly, the diagrams correspond to combinations of the initial and final current states and the occurred virtual currents that transform both states to the vacuum. The weight of each virtual current is $1 / g^{2}$, which is small in the strong coupling regime. Either by interpretation or through managing the relevant terms at a given order of the strong coupling expansion, the diagrams play the role of Feynman diagrams at the small coupling regime. The present expansion of the transfer-matrix elements are used to develop the expansion of the ground state and some excited energies at the lowest orders in the strong coupling regime. Based on the observation that the lattice size dependence of expansions can be factored out from the matrix elements and eigenvalues, the physical interpretation of the results is discussed. 
The existence of the manageable strong coupling expansion, combined with the available perturbative small coupling expansion, might be useful as it could provide some knowledge about the phase structure of the model. A classic example is the 2D Ising model, for which it can be shown that the small coupling (high temperature) expansion of the partition function is equivalent to the strong coupling (low temperature) expansion of the same model but on the dual lattice $[21,22]$. Accordingly, this leads to the fact that the 2D Ising model exhibits a phase transition with a known critical coupling (temperature) [22], even before the exact solution at any coupling is found [23]. These kinds of extra benefits of a strong coupling expansion are especially important in the case of lattice gauge theories, as they are expected to capture the essential features of phase transitions of gauge models. In particular, any relation between the small and the strong coupling expansions may be considered as a piece of evidence for a phase transition in an intermediate coupling value.

\section{ACKNOWLEDGMENTS}

The helpful comments by M. Khorrami on the manuscript are gratefully acknowledged. The authors also would like to thank the anonymous referee for providing detailed and useful comments leading to directions to improve this work. This work is supported by the Research Council of Alzahra University.

\section{APPENDIX A: THIRD AND FOURTH ORDERS OF $\gamma$ IN THE VACUUM BLOCK}

Presenting the contribution at order $\gamma^{3}$ needs a more compact notation, for which we define the new $\Delta$ as following with the summations on all \pm sign combinations

$$
\begin{aligned}
\Delta\left(\frac{\phi_{l} ; M_{l}^{p_{1}}, \ldots, M_{l}^{p_{r}}}{\not q l_{l}^{\prime} ; M_{l}^{p_{1}^{\prime}}, \ldots, M_{l}^{p_{s}^{\prime}}} ; \delta_{l l_{1}}, \ldots, \delta_{l l_{t}}\right)= & \sum_{ \pm} \prod_{l} \delta\left(\not q_{l} \pm M_{l}^{p_{1}} \pm \cdots \pm M_{l}^{p_{r}} \pm \delta_{l l_{1}} \pm \cdots \pm \delta_{l l_{t}}\right) \\
& \times \delta\left(\not \not_{l}^{\prime} \pm M_{l}^{p_{1}^{\prime}} \pm \cdots \pm M_{l}^{p_{s}^{\prime}} \pm \delta_{l l_{1}} \pm \cdots \pm \delta_{l l_{t}}\right)
\end{aligned}
$$

with this caution that the place in lattice and sign of each $\delta_{l l_{i}}$ is the same in both $\delta\left(\not \not_{l} \cdots\right)$ and $\delta\left(\not l_{l}^{\prime} \cdots\right)$, as they come from the term $\cos \left(\theta-\theta^{\prime}\right)$. The total number of terms is then $2^{r+s+t}$. At third order in the vacuum block, we have 80 terms, which may be presented in the compact $\Delta$ notation as

$$
\begin{aligned}
& {\left[\left\langle\boldsymbol{q}^{\prime}|\underline{\hat{V}}| \boldsymbol{q}\right\rangle_{\mathbf{0}}\right]_{\gamma^{3}}=\frac{\gamma^{3}}{384} \sum_{\text {all } p}\left[\Delta\left(\frac{\not_{l}}{\not_{l}^{\prime}, M_{l}^{p_{1}}, M_{l}^{p_{2}}, M_{l}^{p_{3}}}\right)+\Delta\left(\frac{\phi_{l} ; M_{l}^{p_{1}}, M_{l}^{p_{2}}, M_{l}^{p_{3}}}{\not_{l}^{\prime}}\right)\right]} \\
& +\frac{\gamma^{3}}{128} \sum_{\text {all } p}\left[\Delta\left(\frac{\not_{l} ; M_{l}^{p_{1}}}{\not_{l}^{\prime}, M_{l}^{p_{1}^{\prime}}, M_{l}^{p_{2}^{\prime}}}\right)+\Delta\left(\frac{\not_{l} ; M_{l}^{p_{1}}, M_{l}^{p_{2}}}{\not_{l}^{\prime} ; M_{l}^{p_{1}^{\prime}}}\right)\right] \\
& +\frac{\gamma^{3}}{32} \sum_{\text {all } p, l_{1}, l_{2}}\left[\Delta\left(\frac{\phi_{l} ; M_{l}^{p_{1}}}{\phi_{l}^{\prime}} ; \delta_{l l_{1}}, \delta_{l l_{2}}\right)+\Delta\left(\frac{\phi_{l}}{q_{l}^{\prime} ; M_{l}^{p_{1}^{\prime}}} ; \delta_{l l_{1}}, \delta_{l l_{2}}\right)\right] \\
& +\frac{\gamma^{3}}{64} \sum_{\text {all } p, l_{1}}\left[\Delta\left(\frac{\phi_{l} ; M_{l}^{p_{1}}, M_{l}^{p_{2}}}{\phi_{l}^{\prime}} ; \delta_{l l_{1}}\right)+\Delta\left(\frac{\phi_{l}}{\phi_{l}^{\prime} ; M_{l}^{p_{1}^{\prime}}, M_{l}^{p_{2}^{\prime}}} ; \delta_{l l_{1}}\right)\right] \\
& +\frac{\gamma^{3}}{48} \sum_{l_{1}, l_{2}, l_{3}} \Delta\left(\frac{\phi_{l}}{q_{l}^{\prime}} ; \delta_{l l_{1}}, \delta_{l l_{2}}, \delta_{l l_{3}}\right)+\frac{\gamma^{3}}{32} \sum_{\text {all } p, l_{1}} \Delta\left(\frac{\phi_{l} ; M_{l}^{p_{1}}}{q_{l}^{\prime} ; M_{l}^{p_{1}^{\prime}}} ; \delta_{l l_{1}}\right) \text {. }
\end{aligned}
$$


At fourth order having $15 \times 2^{4}=240$ terms, the element in the compact notation comes to the form

$$
\begin{aligned}
& {\left[\left\langle\boldsymbol{q}^{\prime}|\hat{\underline{V}}| \boldsymbol{q}\right\rangle_{\mathbf{0}}\right]_{\gamma^{4}}=\frac{\gamma^{4}}{6144} \sum_{\text {all } p}\left[\Delta\left(\frac{\not \phi_{l}}{\not_{l}^{\prime} ; M_{l}^{p_{1}}, M_{l}^{p_{2}}, M_{l}^{p_{3}}, M_{l}^{p_{4}}}\right)+\Delta\left(\frac{\phi_{l} ; M_{l}^{p_{1}}, M_{l}^{p_{2}}, M_{l}^{p_{3}}, M_{l}^{p_{4}}}{\not_{l}^{\prime}}\right)\right]} \\
& +\frac{\gamma^{4}}{256} \sum_{\text {all } p, l_{1}, l_{2}}\left[\Delta\left(\frac{\phi_{l} ; M_{l}^{p_{1}}, M_{l}^{p_{2}}}{\phi_{l}^{\prime}} ; \delta_{l l_{1}}, \delta_{l l_{2}}\right)+\Delta\left(\frac{\phi_{l}}{\phi_{l}^{\prime} ; M_{l}^{p_{1}}, M_{l}^{p_{2}}} ; \delta_{l l_{1}}, \delta_{l l_{2}}\right)\right] \\
& +\frac{\gamma^{4}}{1024} \sum_{\text {all } p} \Delta\left(\frac{\phi_{l} ; M_{l}^{p_{1}}, M_{l}^{p_{2}}}{\phi_{l}^{\prime} ; M_{l}^{p_{3}}, M_{l}^{p_{4}}}\right)+\frac{\gamma^{4}}{384} \sum_{l_{1}, l_{2}, l_{3}, l_{4}} \Delta\left(\frac{\phi_{l}}{\phi_{l}^{\prime}} ; \delta_{l l_{1}}, \delta_{l l_{2}}, \delta_{l l_{3}}, \delta_{l l_{4}}\right) \\
& +\frac{\gamma^{4}}{1536} \sum_{\text {all } p}\left[\Delta\left(\frac{\phi_{l} ; M_{l}^{p_{1}}, M_{l}^{p_{2}}, M_{l}^{p_{3}}}{\phi_{l}^{\prime} ; M_{l}^{p_{4}}}\right)+\Delta\left(\frac{\phi_{l} ; M_{l}^{p_{1}}}{\phi_{l}^{\prime} ; M_{l}^{p_{2}}, M_{l}^{p_{3}}, M_{l}^{p_{4}}}\right)\right]+\frac{\gamma^{4}}{128} \sum_{\text {all } p, l_{1}, l_{2}} \Delta\left(\frac{\phi_{l} ; M_{l}^{p_{1}}}{\phi_{l}^{\prime} ; M_{l}^{p_{2}}} ; \delta_{l l_{1}}, \delta_{l l_{2}}\right) \\
& +\frac{\gamma^{4}}{768} \sum_{\text {all } p, l_{1}}\left[\Delta\left(\frac{\phi_{l} ; M_{l}^{p_{1}}, M_{l}^{p_{2}}, M_{l}^{p_{3}}}{\phi_{l}^{\prime}} ; \delta_{l l_{1}}\right)+\Delta\left(\frac{\not_{l}}{\not_{l}^{\prime} ; M_{l}^{p_{1}}, M_{l}^{p_{2}}, M_{l}^{p_{3}}} ; \delta_{l l_{1}}\right)\right] \\
& +\frac{\gamma^{4}}{256} \sum_{\text {all } p, l_{1}}\left[\Delta\left(\frac{\not_{l} ; M_{l}^{p_{1}}}{\not_{l}^{\prime} ; M_{l}^{p_{2}}, M_{l}^{p_{3}}} ; \delta_{l l_{1}}\right)+\Delta\left(\frac{\not_{l} ; M_{l}^{p_{1}}, M_{l}^{p_{2}}}{\not_{l}^{\prime} ; M_{l}^{p_{3}}} ; \delta_{l l_{1}}\right)\right] \\
& +\frac{\gamma^{4}}{192} \sum_{p, l_{1}, l_{2}, l_{3}}\left[\Delta\left(\frac{\phi_{l} ; M_{l}^{p}}{\not q_{l}^{\prime}} ; \delta_{l l_{1}}, \delta_{l l_{2}}, \delta_{l l_{3}}\right)+\Delta\left(\frac{\phi_{l}}{\phi_{l}^{\prime} ; M_{l}^{p}} ; \delta_{l l_{1}}, \delta_{l l_{2}}, \delta_{l l_{3}}\right)\right] \text {. }
\end{aligned}
$$

\section{APPENDIX B: NUMERICAL FACTOR}

Here the numerical factor appearing in Eq. (69) is derived. By definitions

$$
\begin{aligned}
A & =\sum_{p} \cos \left(M_{l}^{p} \theta^{l}\right), \\
B & =\sum_{p} \cos \left(M_{l}^{p} \theta^{\prime l}\right), \\
C & =\sum_{l} \cos \left(\theta^{l}-\theta^{\prime l}\right),
\end{aligned}
$$

we can expand the exponent as follows

$$
\begin{aligned}
e^{\gamma\left(\frac{1}{2} A+\frac{1}{2} B+C\right)} & =\sum_{n=0}^{\infty} \frac{\gamma^{n}}{n !} \sum_{\ell=0}^{n} \frac{n !}{\ell !(n-\ell) !}\left(\frac{A}{2}+\frac{B}{2}\right)^{n-\ell} C^{\ell}, \\
& =\sum_{n=0}^{\infty} \frac{\gamma^{n}}{n !} \sum_{\ell=0}^{n} \frac{n !}{\ell !(n-\ell) !} \sum_{m=0}^{n-\ell} \frac{(n-\ell) !}{m !(n-\ell-m) !}\left(\frac{A}{2}\right)^{m}\left(\frac{B}{2}\right)^{n-\ell-m} C^{\ell}, \\
& =\sum_{n=0}^{\infty} \sum_{\ell=0}^{n} \sum_{m=0}^{n-\ell} \frac{\gamma^{n}}{m !(n-\ell-m) ! \ell !}\left(\frac{A}{2}\right)^{m}\left(\frac{B}{2}\right)^{n-\ell-m} C^{\ell} .
\end{aligned}
$$

Setting $n-\ell-m=m^{\prime}$, and changing the phase in Fourier integrals

$$
\cos \alpha=\frac{1}{2}\left(e^{i \alpha}+e^{-i \alpha}\right),
$$


we find the numerical factor in Eq. (69) as

$$
\frac{1}{2^{2 m+2 m^{\prime}+\ell}} \frac{1}{m ! m^{\prime} ! \ell !}
$$

\section{APPENDIX C: MORE BY THE RULES}

As applications of the rules for $\gamma$ expansion of the matrix elements, here more examples are presented.

In vacuum block, we consider the state with two units of currents in the first plaquette, as $\left|\boldsymbol{q}_{\mathbf{2}} \cdot \boldsymbol{M}\right\rangle=|\mathbf{2}\rangle$ with $\boldsymbol{q}_{\mathbf{2}}=(2,0, \ldots, 0)$, represented by $(\mathrm{C} 1)$.

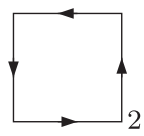

Then, by the rules we find for the matrix element $\langle\mathbf{0}|\underline{\hat{V}}| \mathbf{2}\rangle_{\mathbf{0}}$,

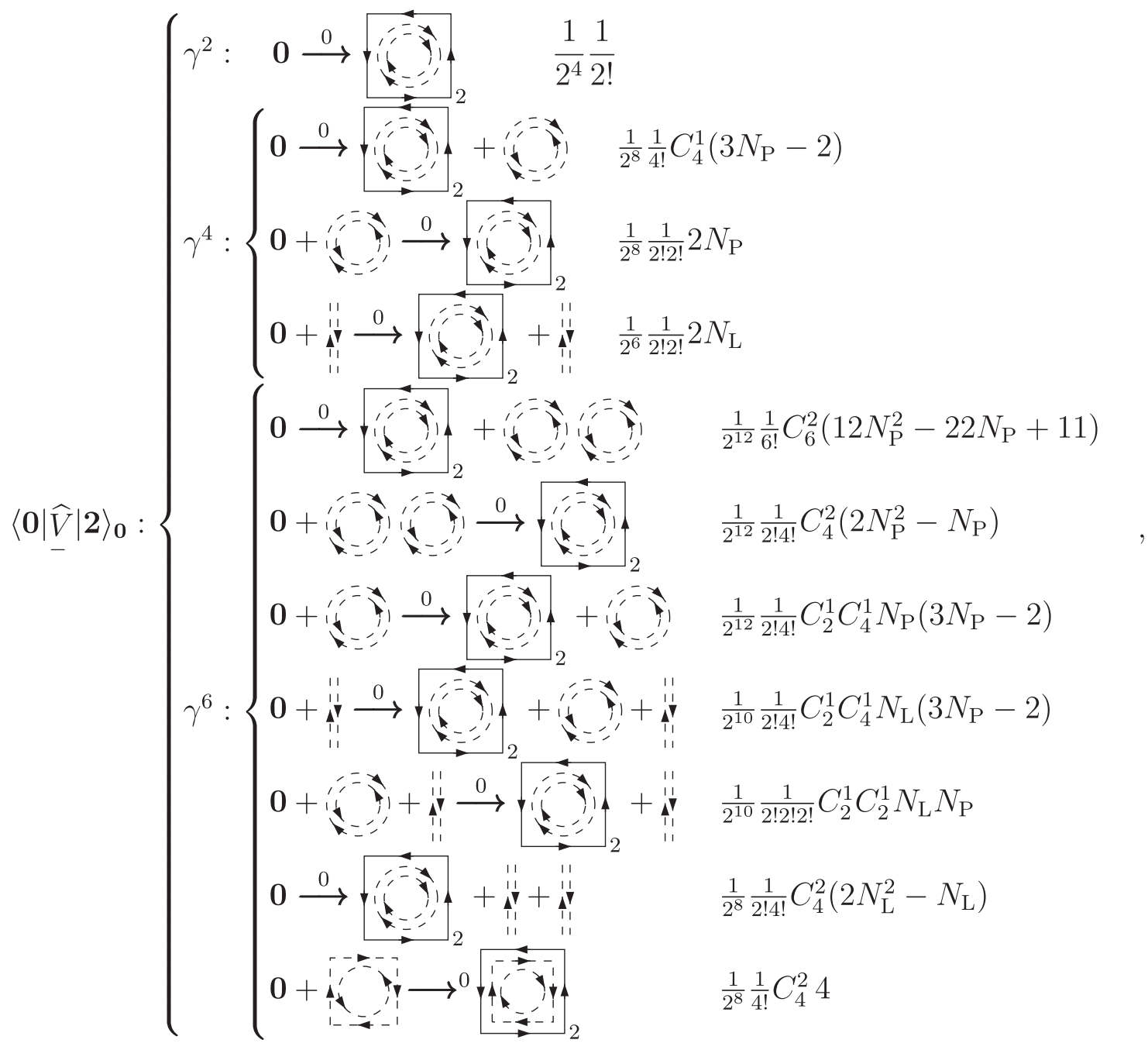

leading to

$$
\langle\mathbf{0}|\underline{\hat{V}}| \mathbf{2}\rangle_{\mathbf{0}}=\frac{\gamma^{2}}{32}+\left(-\frac{1}{768}+\frac{N_{\mathrm{L}}}{128}+\frac{N_{\mathrm{P}}}{256}\right) \gamma^{4}+\left(\frac{779}{196608}-\frac{5 N_{\mathrm{L}}}{6144}+\frac{N_{\mathrm{L}}^{2}}{1024}-\frac{11 N_{\mathrm{P}}}{49152}+\frac{N_{\mathrm{L}} N_{\mathrm{P}}}{1024}+\frac{N_{\mathrm{P}}^{2}}{4096}\right) \gamma^{6}+\cdots
$$


Also for the element below we have

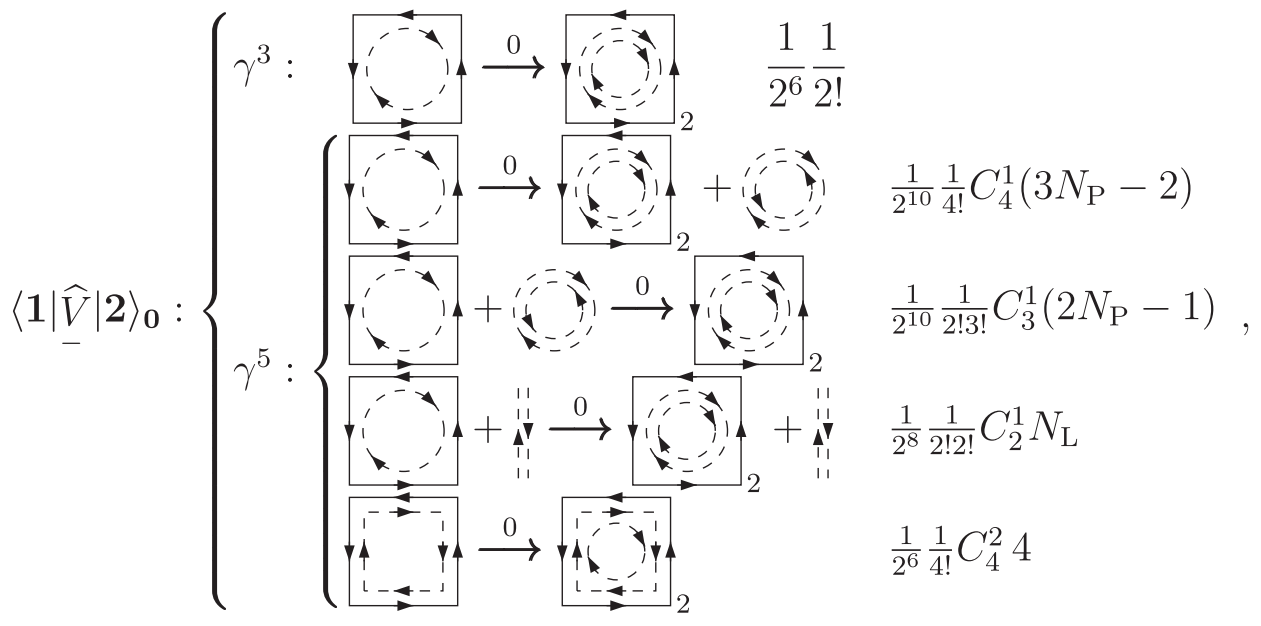

leading to

$$
\langle\mathbf{1}|\underline{\hat{V}}| \mathbf{2}\rangle_{\mathbf{0}}=\frac{\gamma^{3}}{128}+\left(\frac{185}{12288}+\frac{N_{\mathrm{L}}}{512}+\frac{N_{\mathrm{P}}}{1024}\right) \gamma^{5}+\cdots
$$

As the last example by $|\mathbf{2}\rangle$ we consider

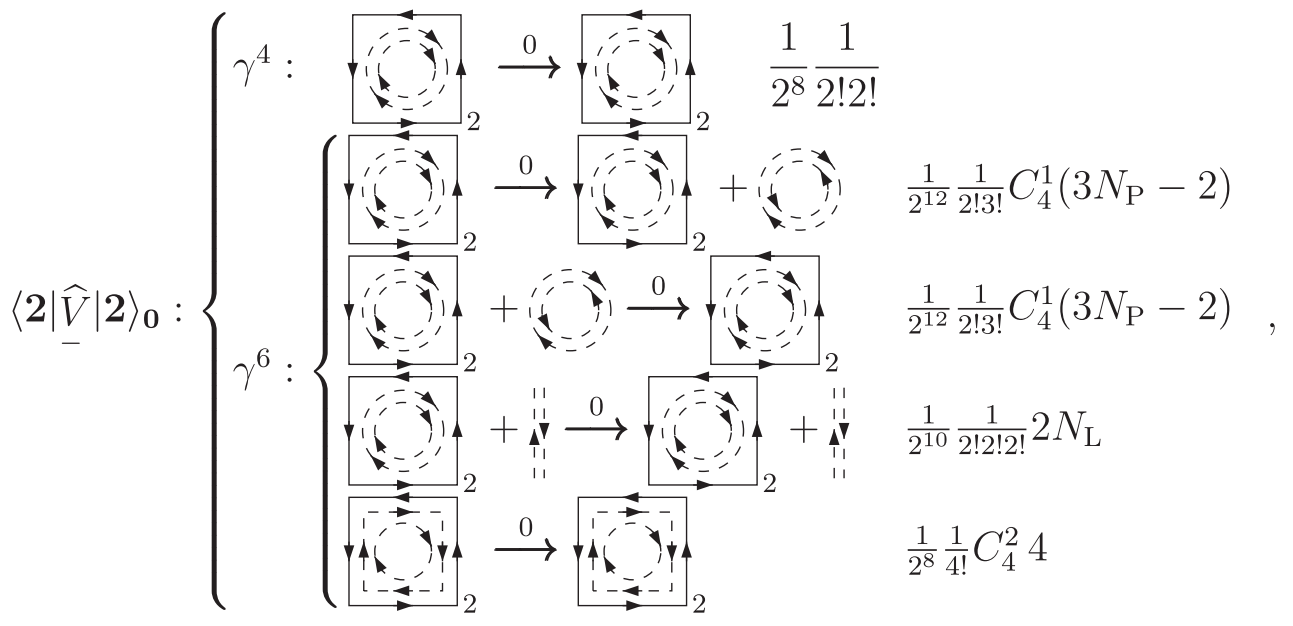

leading to

$$
\langle\mathbf{2}|\hat{\underline{V}}| \mathbf{2}\rangle_{\mathbf{0}}=\frac{\gamma^{4}}{1024}+\left(\frac{47}{12288}+\frac{N_{\mathrm{L}}}{4096}+\frac{N_{\mathrm{P}}}{8192}\right) \gamma^{6}+\cdots .
$$

As the next example, we consider the state with three units of currents in the first plaquette $\left|\boldsymbol{q}_{\mathbf{3}} \cdot \boldsymbol{M}\right\rangle=|\mathbf{3}\rangle$ with $\boldsymbol{q}_{\mathbf{3}}=(3,0, \ldots, 0)$, represented by Eq. (C8).

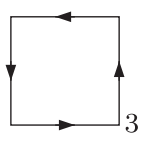

The rules for the matrix element $\langle\mathbf{0}|\underline{\hat{V}}| \mathbf{3}\rangle_{\mathbf{0}}$ give 


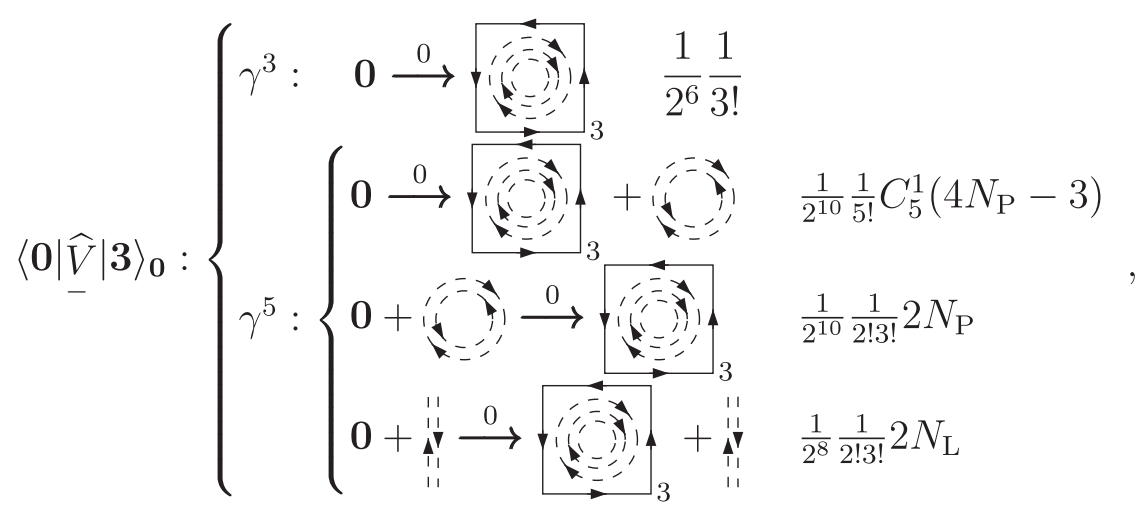

leading to

$$
\langle\mathbf{0}|\underline{\hat{V}}| \mathbf{3}\rangle_{\mathbf{0}}=\frac{\gamma^{3}}{384}+\left(-\frac{1}{8192}+\frac{N_{\mathrm{L}}}{1536}+\frac{N_{\mathrm{P}}}{3072}\right) \gamma^{5}+\cdots
$$

As another example, we consider the state with one unit of current in two neighbor plaquettes, for which we take the first plaquette and its right-side neighbor. By the numbering in Fig. 3 and made as $\left|\boldsymbol{q}_{\mathbf{1 , 1}} \cdot \boldsymbol{M}\right\rangle=|\mathbf{1}, \mathbf{1}\rangle$ with $\boldsymbol{q}_{\mathbf{1}, \mathbf{1}}=(\underbrace{1, \ldots, 0}_{N_{\mathrm{P}}}, 1,0, \ldots, 0)$, this is represented by $(\mathrm{C} 11)$.

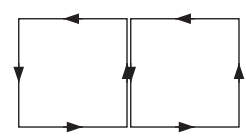

It is obvious that the net current in the shared link is zero. In this case, we have the following:

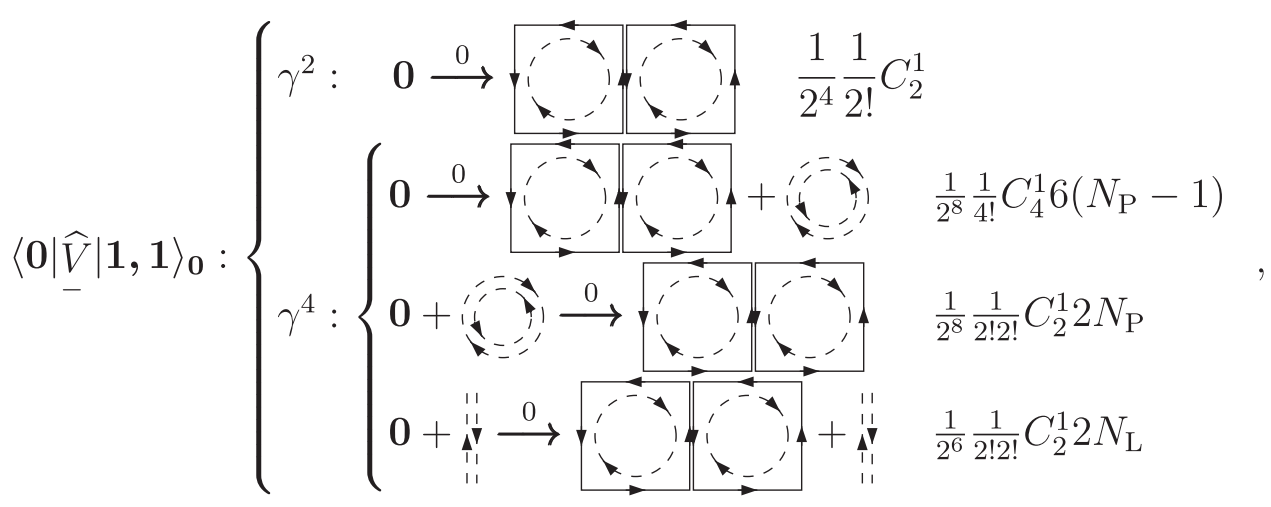

giving

$$
\langle\mathbf{0}|\underline{\hat{V}}| \mathbf{1}, \mathbf{1}\rangle_{\mathbf{0}}=\frac{\gamma^{2}}{16}+\left(-\frac{1}{256}+\frac{N_{\mathrm{L}}}{64}+\frac{N_{\mathrm{P}}}{128}\right) \gamma^{4}+\cdots .
$$

Another example is by the state with one unit of current in the opposite direction of $|\mathbf{1}\rangle$, that is $\left|\boldsymbol{q}_{-\mathbf{1}} \cdot \boldsymbol{M}\right\rangle=|\mathbf{- 1}\rangle$ with $\boldsymbol{q}_{-\mathbf{1}}=(-1,0, \ldots, 0)$, for which, with special care about the direction of arrows, we draw 


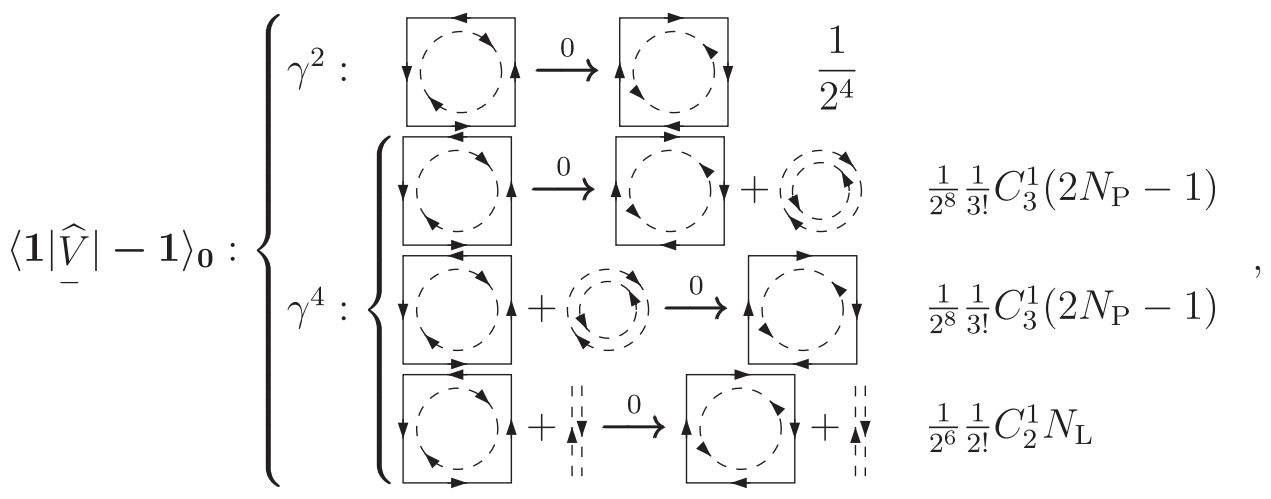

leading to

$$
\langle\mathbf{1}|\underline{\hat{V}}|-\mathbf{1}\rangle_{\mathbf{0}}=\frac{\gamma^{2}}{16}+\left(-\frac{1}{256}+\frac{N_{\mathrm{L}}}{64}+\frac{N_{\mathrm{P}}}{128}\right) \gamma^{4}+\cdots .
$$

As the other example transition between $|\mathbf{1}\rangle$ and one unit of current in another plaquette than the first one, showing as $\left|\mathbf{1}^{\prime}\right\rangle$, is represented as

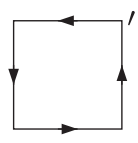

for which we find for the matrix element

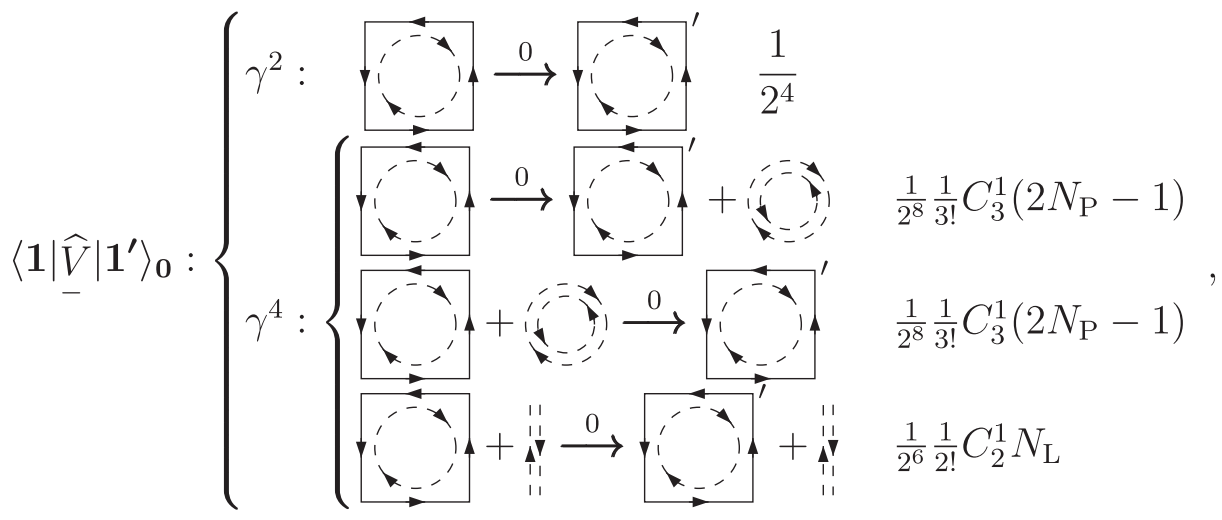

leading to

$$
\left\langle\mathbf{1}|\underline{\hat{V}}| \mathbf{1}^{\prime}\right\rangle_{\mathbf{0}}=\frac{\gamma^{2}}{16}+\left(-\frac{1}{256}+\frac{N_{\mathrm{L}}}{64}+\frac{N_{\mathrm{P}}}{128}\right) \gamma^{4}+\cdots .
$$

As a final example in the vacuum block, we consider $|\mathbf{1}\rangle$ and the state with two units of currents in opposite directions as $|-\mathbf{2}\rangle$, represented by Eq. (C19).

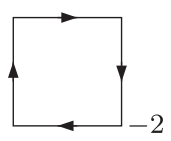

Then the rules, with special care about the direction of arrows, give the following: 


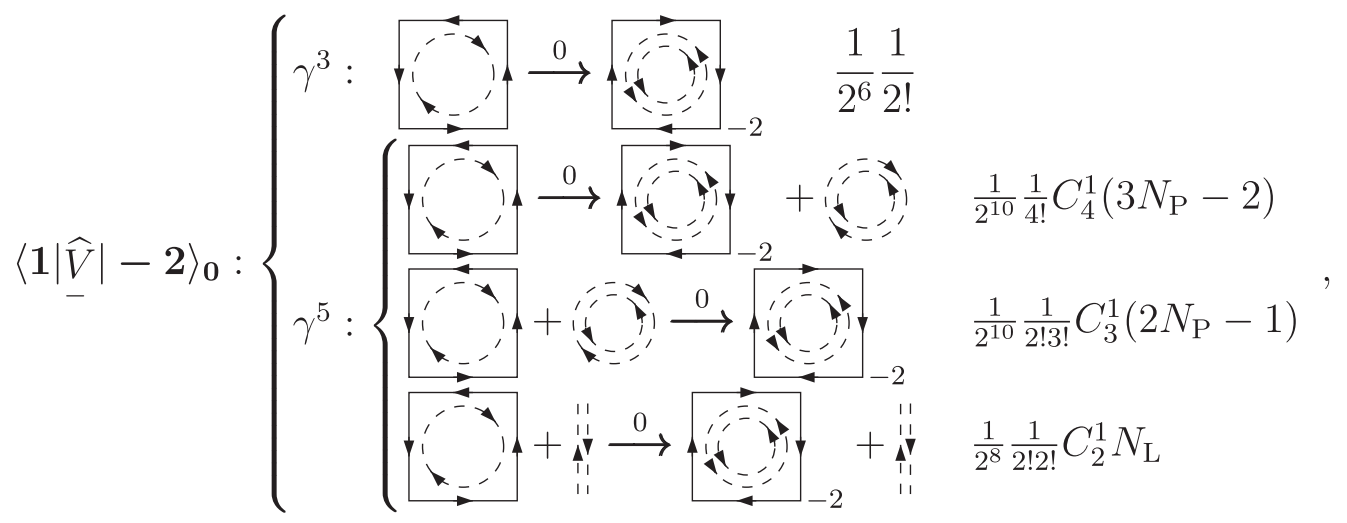

leading to

$$
\langle\mathbf{1}|\underline{\hat{V}}|-\mathbf{2}\rangle_{\mathbf{0}}=\frac{\gamma^{3}}{128}+\left(-\frac{7}{12288}+\frac{N_{\mathrm{L}}}{512}+\frac{N_{\mathrm{P}}}{1024}\right) \gamma^{5}+\cdots .
$$

Samples of the nonvacuum block of $\boldsymbol{k}_{\mathbf{1}}$ are already given. Here as extra examples in the nonvacuum block, first we consider the coblocks of vector $\boldsymbol{k}_{\mathbf{2}}=(2,0, \ldots, 0)$ with two units of currents on the first link. The lowest order of this block occurs when $\boldsymbol{q}^{\prime}=\boldsymbol{q}=\mathbf{0}$, given by

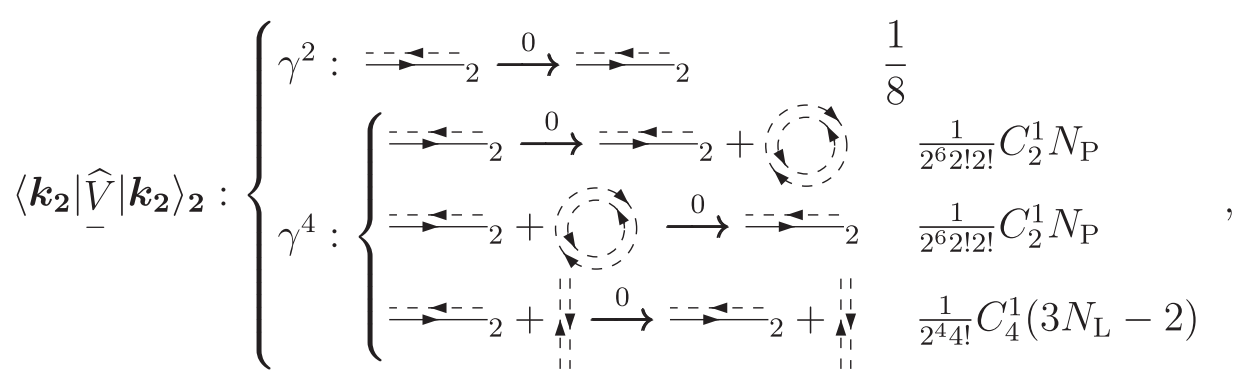

leading to

$$
\left\langle\boldsymbol{k}_{\mathbf{2}}|\hat{\underline{V}}| \boldsymbol{k}_{\mathbf{2}}\right\rangle_{\mathbf{2}}=\frac{\gamma^{2}}{8}+\left(-\frac{1}{48}+\frac{N_{\mathrm{L}}}{32}+\frac{N_{\mathrm{P}}}{64}\right) \gamma^{4}+\cdots .
$$

As a coblock of $\boldsymbol{k}_{\mathbf{2}}$ we consider $\boldsymbol{k}_{\mathbf{2} ; \mathbf{1}}=\boldsymbol{k}_{\mathbf{2}}+\not \phi_{\mathbf{1}}$, which has three units of currents on the first link, and two units of currents on other links of the first plaquette. Similar to Eq. (62) we may represent it as (C24).

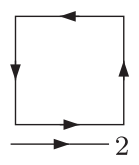

The graphical representation for $\boldsymbol{q}^{\prime}=\mathbf{0}$ and $\boldsymbol{q}=\boldsymbol{q}_{\mathbf{1}}$ will be given as follows: 


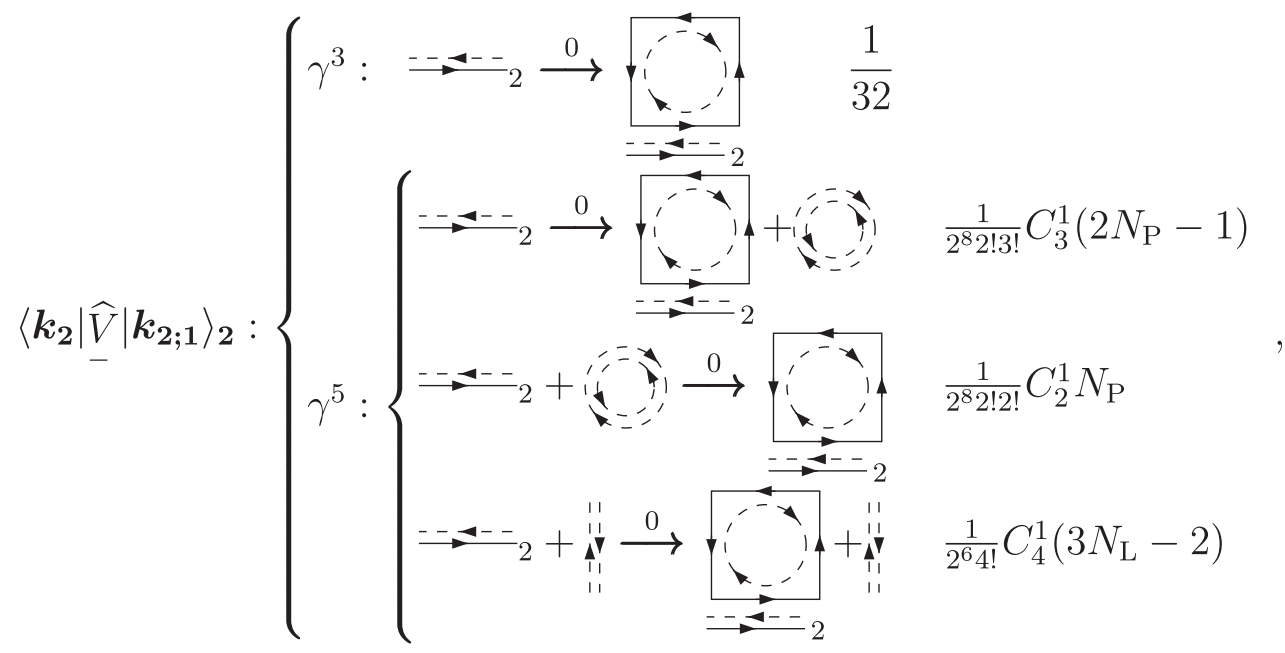

all together leading to

$$
\left\langle\boldsymbol{k}_{\mathbf{2}}|\hat{\underline{V}}| \boldsymbol{k}_{\mathbf{2} ; \mathbf{1}}\right\rangle_{\mathbf{2}}=\frac{\gamma^{3}}{32}+\left(-\frac{19}{3072}+\frac{N_{\mathrm{L}}}{128}+\frac{N_{\mathrm{P}}}{256}\right) \gamma^{5}+\cdots
$$

The graphical representation for the last example of this nonvacuum block is $\boldsymbol{q}^{\prime}=\boldsymbol{q}=\boldsymbol{q}_{\mathbf{1}}$ :

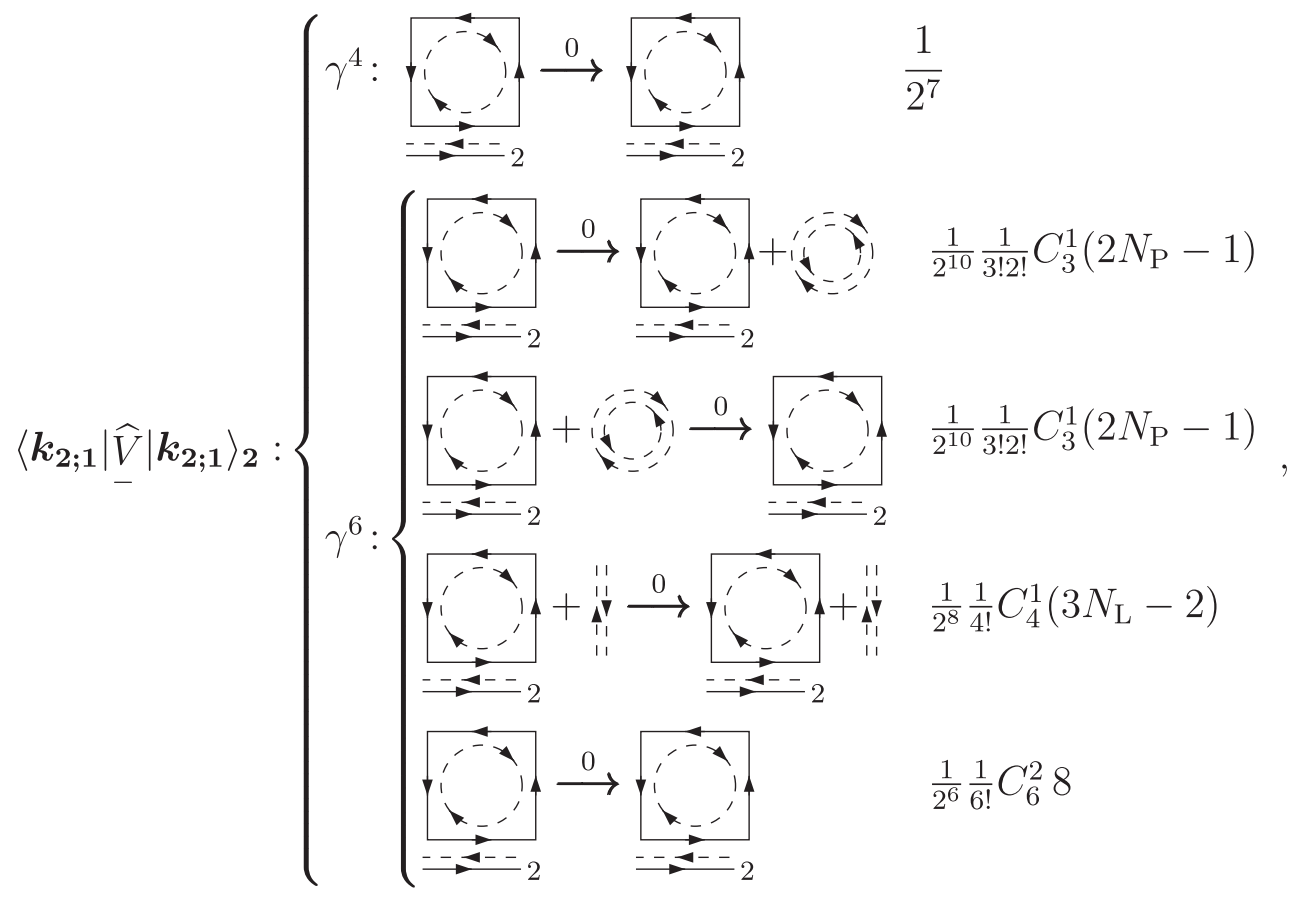

leading to

$$
\left\langle\boldsymbol{k}_{\mathbf{2} ; \mathbf{1}}|\underline{\hat{V}}| \boldsymbol{k}_{\mathbf{2} ; \mathbf{1}}\right\rangle_{\mathbf{2}}=\frac{\gamma^{4}}{128}+\left(\frac{5}{6144}+\frac{N_{\mathrm{L}}}{512}+\frac{N_{\mathrm{P}}}{1024}\right) \gamma^{6}+\cdots
$$

As the last example, we consider the block by the representative state $\boldsymbol{k}_{\mathbf{1}, \mathbf{1}^{\prime}}$ in which two separated links have one unit of currents, showing them as (C29).

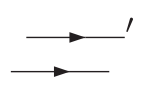


Then by the rules we have

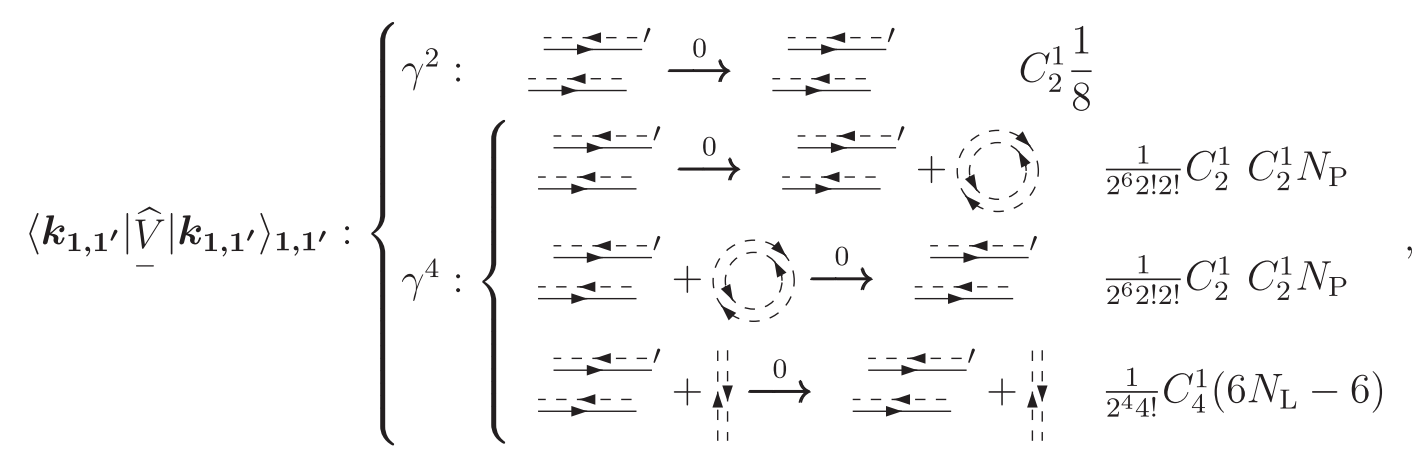

leading to

$$
\left\langle\boldsymbol{k}_{\mathbf{1 , \mathbf { 1 } ^ { \prime }}}|\hat{\underline{V}}| \boldsymbol{k}_{\mathbf{1 , \mathbf { 1 } ^ { \prime }}}\right\rangle_{\mathbf{1}, \mathbf{1}^{\prime}}=\frac{\gamma^{2}}{4}+\left(-\frac{1}{16}+\frac{N_{\mathrm{L}}}{16}+\frac{N_{\mathrm{P}}}{32}\right) \gamma^{4}+\cdots
$$

[1] R. P. Feynman, Space-time approach to quantum electrodynamics, Phys. Rev. 76, 769 (1949).

[2] T. Aoyama, M. Hayakawa, T. Kinoshita, and M. Nio, Quantum electrodynamics calculation of lepton anomalous magnetic moments: Numerical approach to the perturbation theory of QED, Prog. Theor. Exp. Phys. 2012, $01 \mathrm{~A} 107$ (2012).

[3] K. G. Wilson, Confinement of quarks, Phys. Rev. D 10, 2445 (1974).

[4] J. B. Kogut, An introduction to lattice gauge theory and spin systems, Rev. Mod. Phys. 51, 659 (1979).

[5] R. Savit, Topological Excitations in U(1)-Invariant Theories, Phys. Rev. Lett. 39, 55 (1977).

[6] T. Banks, R. Myerson, and J. B. Kogut, Phase transitions in Abelian lattice gauge theories, Nucl. Phys. B129, 493 (1977).

[7] J. Villain, Theory of one- and two-dimensional magnets with an easy magnetization plane II. The planar, classical, two-dimensional magnet, J. Phys. II (France) 36, 581 (1975).

[8] C. Gattringer, New developments for dual methods in lattice field theory at non-zero density, Proc. Sci. LATTICE2013 (2014) 002.

[9] M. Zach, M. Faber, and P. Skala, Investigating confinement in dually transformed U(1) lattice gauge theory, Phys. Rev. D 57, 123 (1998).

[10] M. Zach, M. Faber, and P. Skala, Flux tubes and their interaction in U(1) lattice gauge theory, Nucl. Phys. B529, 505 (1998).

[11] M. Panero, A numerical study of confinement in compact QED, J. High Energy Phys. 05 (2005) 066.
[12] O. Borisenko, V. Chelnokov, M. Gravina, and A. Papa, Deconfinement and universality in the 3D U(1) lattice gauge theory at finite temperature: Study in the dual formulation, J. High Energy Phys. 09 (2015) 062.

[13] M. Caselle, M. Panero, and D. Vadacchino, Width of the flux tube in compact $\mathrm{U}(1)$ gauge theory in three dimensions, J. High Energy Phys. 02 (2016) 180.

[14] N. Vadood and A. H. Fatollahi, On U(1) gauge theory transfer-matrix in fourier basis, Commun. Theor. Phys. 71, 921 (2019).

[15] M. Luscher, Construction of a selfadjoint, strictly positive transfer matrix for euclidean lattice gauge theories, Commun. Math. Phys. 54, 283 (1977).

[16] K. Osterwalder and E. Seiler, Gauge field theories on a lattice, Ann. Phys. (N.Y.) 110, 440 (1978).

[17] N. Vadood and A. H. Fatollahi, Lost in normalization, Europhys. Lett. 131, 41003 (2020).

[18] J. M. Jauch and F. Rohrlich, The Theory of Photons and Electrons, 2nd edition (Springer, New York, 1980), Ch. 9.

[19] M.E. Peskin and D. V. Schroeder, An Introduction to Quantum Field Theory (CRC Press, Boca Raton, 2018), Ch. 4.

[20] L. H. Ryder, Quantum Field Theory, 2nd ed. (Cambridge University Press, Cambridge, England, 1996), Ch. 6.

[21] K. Huang, Statistical Mechanics, 2nd ed. (Wiley, New York, 1987).

[22] H. A. Kramers and G. H. Wannier, Statistics of the twodimensional ferromagnet. Part I, Phys. Rev. 60, 252 (1941).

[23] L. Onsager, Crystal statistics. I. A two-dimensional model with an order-disorder transition, Phys. Rev. 65, 117 (1944). 